University of Louisville

ThinkIR: The University of Louisville's Institutional Repository

Electronic Theses and Dissertations

$12-2017$

\title{
Numerical investigation of drop impingement on dry and wet surfaces.
}

Yisen Guo

University of Louisville

Follow this and additional works at: https://ir.library.louisville.edu/etd

Part of the Fluid Dynamics Commons

\section{Recommended Citation}

Guo, Yisen, "Numerical investigation of drop impingement on dry and wet surfaces." (2017). Electronic Theses and Dissertations. Paper 2869.

https://doi.org/10.18297/etd/2869

This Doctoral Dissertation is brought to you for free and open access by ThinkIR: The University of Louisville's Institutional Repository. It has been accepted for inclusion in Electronic Theses and Dissertations by an authorized administrator of ThinkIR: The University of Louisville's Institutional Repository. This title appears here courtesy of the author, who has retained all other copyrights. For more information, please contact thinkir@louisville.edu. 


\title{
NUMERICAL INVESTIGATION OF DROP IMPINGEMENT ON DRY AND WET SURFACES
}

\author{
By \\ Yisen Guo \\ B.S., Shandong University, 2009 \\ M.S., Oregon State University, 2011
}

\begin{abstract}
A Dissertation
Submitted to the Faculty of the

J.B. Speed School of Engineering of the University of Louisville in Partial Fulfillment of the Requirements

for the Degree of

Doctor of Philosophy

in Mechanical Engineering

Department of Mechanical Engineering

University of Louisville

Louisville, Kentucky
\end{abstract}

December 2017 
Copyright 2017 by Yisen Guo

All rights reserved 



\title{
NUMERICAL INVESTIGATION OF DROP IMPINGEMENT ON DRY AND WET SURFACES
}

\author{
By \\ Yisen Guo \\ B.S., Shandong University, 2009 \\ M.S., Oregon State University, 2011
}

A Dissertation Approved on

November 1, 2017

by the following Dissertation Committee:

$\overline{\text { Yongsheng Lian, Ph.D., Dissertation Director }}$

Dar-Jen Chang, Ph.D.

Kevin Murphy, Ph.D.

Keith Sharp, Ph.D. 


\section{ACKNOWLEDGMENTS}

I would like to express my gratitude to my advisor, Dr. Yongsheng Lian. Thank you for your immense support over the years; your guidance and encouragement were invaluable. You introduced me into the world of multiphase flow simulations and taught me to think critically about my research. You were always patient explaining complicated concepts in a gentle way. You always gave me constructive suggestions and great comments about the simulations when I struggled. And your great sense of humor is contagious, I believe I am becoming a more optimistic person. I consider myself very lucky to have the opportunity to work in your group and I have truly enjoyed working with you!

Thank you to Dr. Keith Sharp, Dr. Dar-Jen Chang and Dr. Kevin Murphy, for serving as my committee members.

Thank you to the group members and many friends I've met in Louisville, for such a pleasant graduate school experience.

Thank you to my wife and my parents, for your love and support; and thank you to my son, even though you are only 3-month old and haven't really done anything to support me. 


\begin{abstract}
NUMERICAL INVESTIGATION OF DROP IMPINGEMENT ON DRY AND WET SURFACES
\end{abstract}

Yisen Guo

November, 12017

In-flight icing due to supercooled large droplets (SLDs) imposes great danger on aviation safety. Because of the large size, SLDs have different characteristics than typical cloud droplets that most icing encounters involve. As a result, SLDs more likely hit the wing surface and move into areas not protected by de-icing devices to form ice, which can lead to loss of lift, increase in drag, altered controllability, and eventually stall and loss of control of the aircraft. The phenomenon of droplet splashing is considered as the most important aspect of the SLD icing accretion problem. Although previous studies have established splashing threshold and revealed most parameters affecting splashing, the splashing mechanism is still not fully understood.

In this study, the impacts of droplets on both dry and wet surfaces were numerically investigated to understand the splashing mechanism. A Navier-Stokes solver was used to describe the flow field, the moment-of-fluid (MOF) method was used to capture the droplet interface evolution, the adaptive mesh refinement technique was employed to refine the mesh near the regions of interest, and the dynamic contact angle model was used to represent the wettability of the solid surface. 
Using the multiphase flow solver, the effect of ambient gas density on splashing was intensively studied and it was confirmed that lowering ambient gas density can suppress dry surface splashing while has no significant influence on wet surface splashing. Then, high-speed drop impact on thin liquid film with a focus on oblique impact was investigated. The numerical results showed that the tangential velocity can significantly alter impact phenomena: a higher tangential velocity leads to lower lamella height and radius on the side behind the advancing drop, the higher tangential velocity also leads to stronger vortices at the drop and film interface due to Kelvin-Helmholtz instability. Simulations of oblique impacts of two neighboring drops were also conducted for low-speed and highspeed impacts. Strong interaction occurred when the crowns formed by the two neighboring drops interfered with each other. For low-speed impact, droplets deposited on to the liquid film with short and thick crowns formed and the interaction region was superposition of crowns. For high-speed impact, crowns broken up to form splashing and the interaction behavior became complicated.

Finally, the droplet impacts on a MS(1)-317 airfoil was studied and the water collection efficiency and impingement limit were investigated. Unlike most previous studies, the flow field and droplet behavior were simultaneously simulated using one multiphase flow solver. The results were compared with the ice accretion simulation code LEWICE and experimental data. The simulations showed that the calculated water collection efficiency of cloud-sized droplets matched the result of LEWICE, however, the calculated water collection efficiency of large droplets showed better agreement with the experimental data than LEWICE. The better agreement was attributed to the droplet trajectory calculation and the capture of droplet splashing. 


\section{TABLE OF CONTENTS}

ACKNOWLEDGMENTS ................................................................................. ii

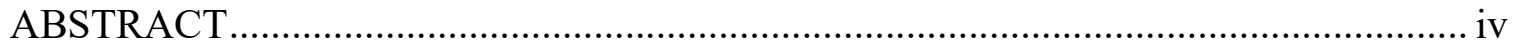

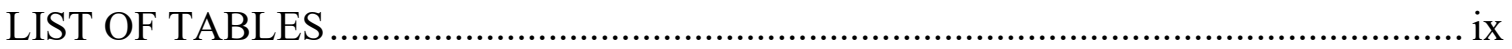

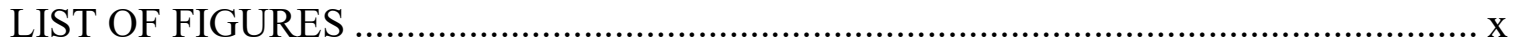

CHAPTER 1. GENERAL INTRODUCTION ….................................................... 1

1.1 Aircraft Icing Due to SLDs .................................................................. 1

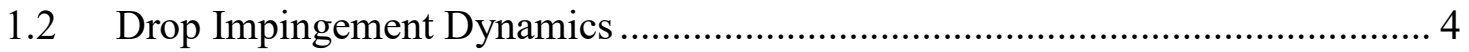

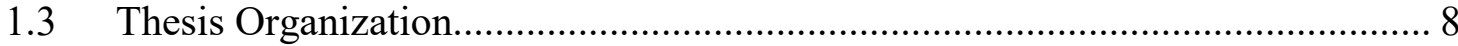

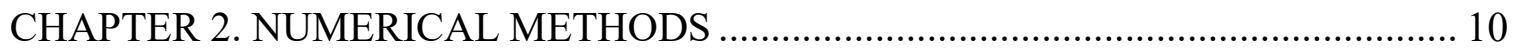

2.1 Governing Equations ......................................................................... 10

2.2 Moment-of-fluid interface reconstruction................................................ 11

2.3 Moment-of-fluid interface advection .......................................................... 15

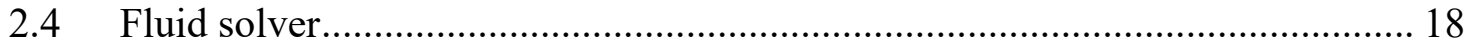

2.5 Dynamic contact angle models .............................................................. 23

CHAPTER 3. DROP IMPACT ON DRY AND WET SURFACES WITH

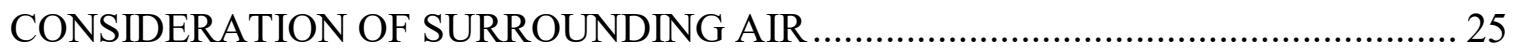




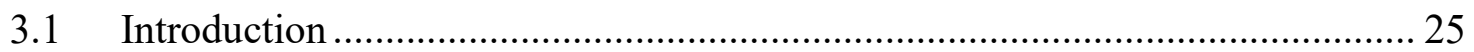

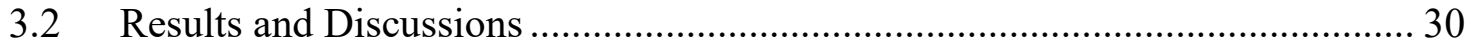

3.2.1 Code validation 1: drop deposition on a hydrophilic surface ...................... 31

3.2.2 Code validation 2: drop complete rebound on a hydrophobic surface ....... 34

3.2.3 Code validation 3: drop impact on a thin liquid film.................................. 35

3.2.4 Ambient air effect on low-speed drop impact on dry surfaces ................... 39

3.2.5 The effect of ambient air density on high speed drop impact..................... 46

3.2.6 Ambient air effect on drop impact on thin liquid film................................ 49

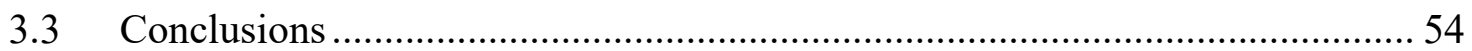

CHAPTER 4. HIGH-SPEED OBLIQUE DROP IMPACT ON THIN LIQUID FILMS. 56

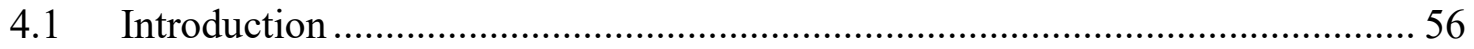

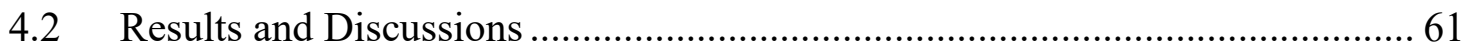

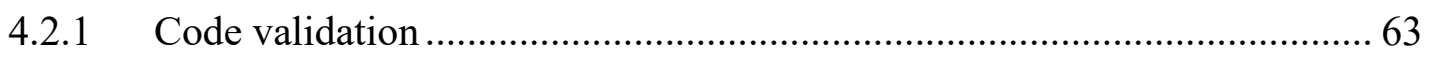

4.2.2 Effect of impact angle ........................................................................ 72

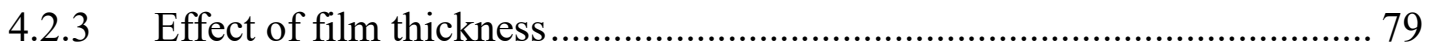

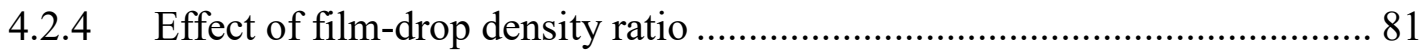

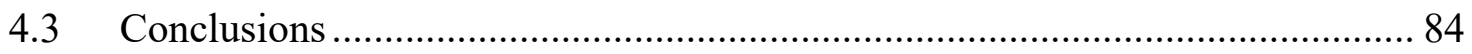

CHAPTER 5. IMPACT OF MULTIPLE DROPS ON THIN LIQUID FILM................... 86

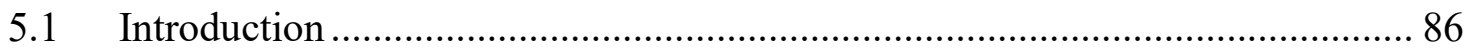

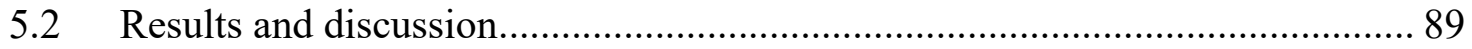


5.2.1 Code validation: Normal impact on liquid film 89

5.2.2 Oblique impact of multiple drops on a thin film 90

5.3 Conclusions 97

CHAPTER 6. CALCULATION OF WATER COLLECTION EFFICIENCY 99

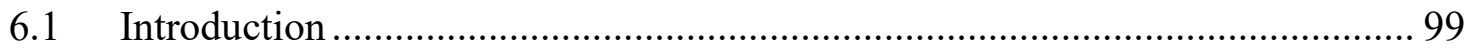

6.1.1 Water collection efficiency and its importance .................................. 99

6.1.2 Existing methods to predict water collection efficiency........................ 100

6.1.3 Effect of large droplets on water collection efficiency .......................... 103

6.2 Procedure of calculating water collection efficiency ................................. 104

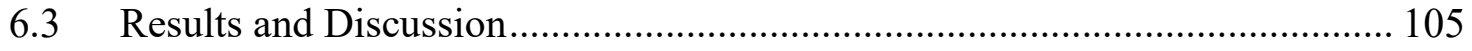

6.3.1 Code validation: droplet impact on dry surface .................................... 105

6.3.2 High-speed normal impact on a dry surface ...................................... 108

6.3.3 Droplet impact on thin liquid film .................................................. 110

6.3.4 Code validation: water collection efficiency calculation for a sphere...... 112

6.3.5 Water collection efficiency calculation for an MS(1)-317 airfoil ............ 115

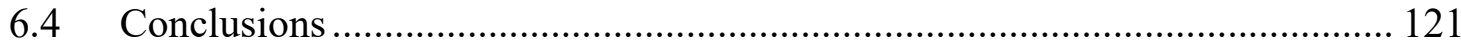

CHAPTER 7. GENERAL CONCLUSIONS ........................................................ 122

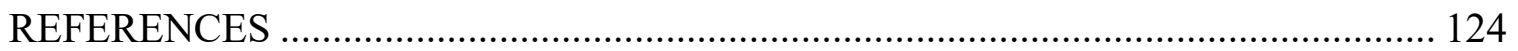

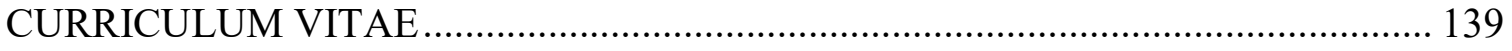




\section{LIST OF TABLES}

Table 1. Summary of the parameters in numerical simulations.................................... 31

Table 2. Selected splash threshold models for drop impact on liquid film.....................59

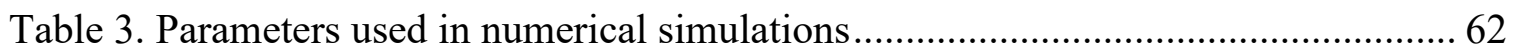

Table 4. Selected existing models for calculating water collection efficiency ............... 102

Table 5. Discretized droplet distributions for MVD of $18.6 \mu \mathrm{m}$................................ 114

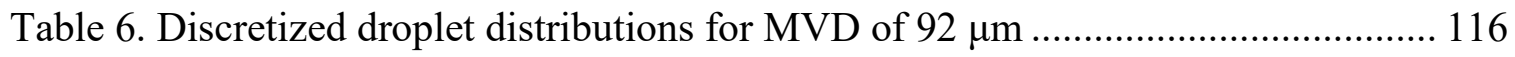




\section{LIST OF FIGURES}

Figure 1. Characteristics of supercooled large droplet. (a) SLDs more likely hit the wing surface and move into areas not protected by de-icing devices to form ice; (b) typical cloud droplets usually follow the streamlines and likely hit the leading edge which is protected by de-icing devices (Protected area is shown in red) ............................................... 2 Figure 2. The interface can be represented by a straight line in $2 \mathrm{D}$ case. The square

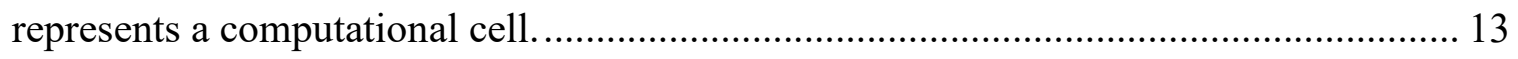

Figure 3. MOF interface reconstruction. The solid curved line represents the true interface and the dashed straight line represents the reconstructed interface.

Figure 4. Illustration of backward tracking process. The dashed region in (b) represents the departure region of cell $\mathrm{i}, \Omega_{i}{ }^{D}$; the dashed square in $(\mathrm{a}), \Omega_{\mathrm{i}}$, is the target region. The shaded region in (b), $\Omega_{m, i}^{D}=V_{-1, i}^{n} \cup V_{0, i}^{n}$, is the material $\mathrm{m}$, in the departure region. The shaded region in (a), $\Omega_{m, i}^{T}=V_{-1, i}^{n+1} \cup V_{0, i}^{n+1}$, is the material $\mathrm{m}$, in the target region. 16

Figure 5. Three-level adaptive mesh refinement based on triple point and curvature of the interface in a two-dimensional simulation. 22

Figure 6. Two-level adaptive mesh refinement in a three-dimensional simulation 22 Figure 7. Comparison of three dynamic contact angle models vs. the Capillary number. 24 Figure 8. Comparison of drop shapes during the impact process. Numerical results are shown in green and experimental results (Kim \& Chun, 2001) in black 33 
Figure 9. Comparison of wetted surface diameter between the experiment and simulation.

Figure 10. Comparison of the drop shapes in drop complete rebound phenomenon. Top: experiment by Azimi et al. (Azimi et al., 2013). Bottom: simulation. 35

Figure 11. 2D axisymmetric simulation of ethanol drop impacts on a $30 \mu \mathrm{m}$ ethanol film with drop dimeter of $271 \mu \mathrm{m}$ and impact velocity of $12.5 \mathrm{~m} / \mathrm{s}(\mathrm{We}=1500, \operatorname{Re}=2227) .36$ Figure 12. 3D simulation of ethanol drop impacts on a $30 \mu \mathrm{m}$ ethanol film with drop dimeter of $271 \mu \mathrm{m}$ and impact velocity of $12.5 \mathrm{~m} / \mathrm{s}(\mathrm{We}=1500, \mathrm{Re}=2227)$. 37 Figure 13. Comparison of the radius of crown's rim in ethanol drop impact on thin liquid film. 39

Figure 14. Droplet splashing on a solid surface. Red: numerical simulation; Black: experimental results ( $\mathrm{Xu}$ et al., 2005). $\mathrm{We}=1685, \mathrm{Re}=8361$, and $\mathrm{K}=393$.

Figure 15. Close-up look of the trapped air and the lamella. The trapped air is not responsible for the splashing.

Figure 16. Comparison of droplet impact at $\rho_{\text {air }}=1.225 \mathrm{~kg} / \mathrm{m}^{3}$ and $0.245 \mathrm{~kg} / \mathrm{m}^{3}$. Splash occurs at high ambient air density but not at low density. 43

Figure 17 . Vorticity contours near the lamella at $\rho_{\text {air }}=1.225 \mathrm{~kg} / \mathrm{m}^{3}$ and $0.245 \mathrm{~kg} / \mathrm{m}^{3} \ldots .44$ Figure 18. Development of a recirculation region inside the drop at $\rho_{\text {air }}=1.225 \mathrm{~kg} / \mathrm{m}^{3} . .45$ Figure 19. Air bubble is trapped at the center of the drop at $\mathrm{t}=0.12 \mathrm{~ms}$ for drop impact at $\rho_{\text {air }}=1.225 \mathrm{~kg} / \mathrm{m}^{3}$. The interface between liquid and gas is shown by dashed red lines. . 45 Figure 20. Development of recirculation region for drop impact at $\rho_{\text {air }}=0.245 \mathrm{~kg} / \mathrm{m}^{3} \ldots 46$ Figure 21. Comparison of $200-\mu \mathrm{m}$ and $20-\mu \mathrm{m}$ droplet impact at $\rho_{\text {air }}=1.225 \mathrm{~kg} / \mathrm{m}^{3} \ldots \ldots . .48$ Figure 22. Comparison of $200-\mu \mathrm{m}$ and $20-\mu \mathrm{m}$ droplet impact at $\rho_{\text {air }}=0.49 \mathrm{~kg} / \mathrm{m}^{3} \ldots \ldots \ldots . .48$ 
Figure 23. 3D simulation of $200-\mu \mathrm{m}$ droplet impact at $\rho_{\text {air }}=1.225 \mathrm{~kg} / \mathrm{m}^{3}$

Figure 24. Comparison of droplet impact on thin liquid film at different ambient air densities 51

Figure 25. Comparison of the radius of crown's rim at different air densities with theoretical solution 51

Figure 26. Comparison of mass loss from splashing with empirical models. 53 Figure 27. 3D simulation of droplet impact on thin liquid film. 54 Figure 28. Selected splash threshold models for water drops impact on liquid films of $\delta=$ 0.1. The abscissa represents drop diameter and the ordinate represents the corresponding threshold velocity for splash. 59

Figure 29. Illustration of the simulation setup of a liquid drop impacting onto thin liquid film. 63

Figure 30. Convergence of crown radius at $\mathrm{T}=1$. The effective grid resolutions are 40, 52, 62 and 74 cells per diameter. The percentages show the dimensionless crown radius increase when increasing grid resolution. 64 Figure 31. Water drop normal impact onto a $2.57 \mathrm{~mm}$ thick water film with the drop dimeter of $3.82 \mathrm{~mm}$ and terminal impact velocity of $3.94 \mathrm{~m} / \mathrm{s}(\mathrm{We}=842$ and $\mathrm{Re}=15,366)$. (a): experiment (Cossali et al., 2004); (b): simulation. 66 Figure 32. Time evolutions of crown diameters of the impact of a 3.82-mm-diameter water drop onto a $2.57-\mathrm{mm}$-thick water film with the impact velocity of $3.51 \mathrm{~m} / \mathrm{s}(\mathrm{We}=667, \mathrm{Re}$ $=13,676$, and $\delta=0.67)$. The simulation result $(\boldsymbol{\nabla})$ shows good agreement with the experimental measurement $(\triangle)($ Cossali et al., 2004) 
Figure 33. Validation with experiment by Zhang et al. (Zhang et al., 2012). We $=324$, Re $=2191$ and $\delta=0.2$. The experimental image and our numerical simulation are taken at $\mathrm{t}=$ $0.335 \mu$ s and $0.331 \mu \mathrm{s}$, respectively. (a): numerical simulation showing drop in red color, liquid film interface in black line, and trapped air bubbles in blue color. (b): liquid surface profile comparison of experiment and simulation (red line). 68

Figure 34. Normal impact of a 41.3- $\mu$ m-diameter water drop on a 1- $\mu$ m-thickness water film (left) and a $5-\mu \mathrm{m}$-thickness water film (right). Normal impact velocity is $53 \mathrm{~m} / \mathrm{s} . . .71$ Figure 35. Time evolutions of crown radius, $r_{c}$, and crown height, $h_{c}$. The numerical simulations are shown in Figure 34. $\mathrm{D}_{0}=41.3 \mu \mathrm{m}, \mathrm{V}_{0}=53 \mathrm{~m} / \mathrm{s}, \mathrm{h}_{0}=1 \mu \mathrm{m}$ and $5 \mu \mathrm{m} . . .72$ Figure 36. Oblique impact of 41.3- $\mu$ m-diameter water drop on 5- $\mu \mathrm{m}$-thick water film. Left: impact angle is $60^{\circ}\left(\mathrm{V}_{0 \mathrm{n}}=53 \mathrm{~m} / \mathrm{s}\right.$ and $\left.\mathrm{V}_{0 \mathrm{t}}=30.6 \mathrm{~m} / \mathrm{s}\right)$. Right: impact angle is $30^{\circ}\left(\mathrm{V}_{0 \mathrm{n}}=\right.$

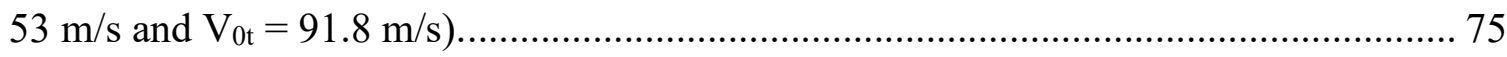

Figure 37. Zoomed-in view of the area bounded by the dashed red rectangle in Figure 36. As the drop impacts onto the film it rolls over the film and then re-impinges on the surrounding quiescent film. 76

Figure 38. Time evolutions of crown radius, $\mathrm{r}_{\mathrm{c}}$, and crown height, $\mathrm{h}_{\mathrm{c}}$, on the downstream side. 77

Figure 39. Two-dimensional simulations of oblique impact of a 41.3- $\mu$ m-diameter water drop on a $5-\mu$ m-thick water film. White represents drop and red represents film. Left: impact angle is $60^{\circ}$. Right: impact angle is $30^{\circ}$ 78 Figure 40. Oblique impact of $41.3-\mu \mathrm{m}$-diameter water drop on 5 - $\mu \mathrm{m}$-thick water film. Impact angle is $15^{\circ}\left(\mathrm{V}_{0 \mathrm{n}}=53 \mathrm{~m} / \mathrm{s}\right.$ and $\left.\mathrm{V}_{0 \mathrm{t}}=197.8 \mathrm{~m} / \mathrm{s}\right)$. 79 
Figure 41. Oblique impacts of 41.3- $\mu \mathrm{m}$ water drops on water films of $1 \mu \mathrm{m}, 5 \mu \mathrm{m}$ and 20

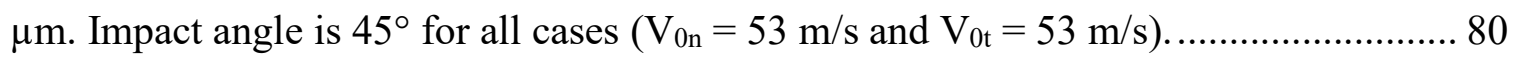
Figure 42. Normal impact of 41.3- $\mu \mathrm{m}$ water drop on $5-\mu \mathrm{m}$ liquid films with $53 \mathrm{~m} / \mathrm{s}$ impact

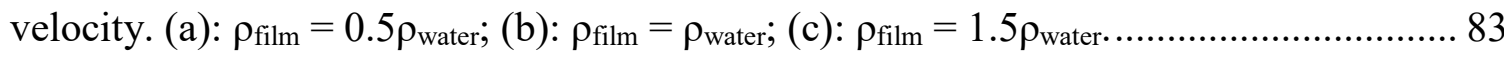
Figure 43. Normal impact of 2-mm-diameter glycerin-water droplet on same liquid film at $2.17 \mathrm{~m} / \mathrm{s}$. Top row: liquid film thickness is $0.2 \mathrm{~mm}$. Bottom row: liquid film thickness is 2 mm. Experiments were performed by Vander Wal et al. (Vander Wal et al., 2006)....... 89 Figure 44. Simulation setup of two neighboring droplets oblique impact on a liquid film. 91

Figure 45. Oblique impact of two neighboring water droplets impact on a 5- $\mu \mathrm{m}$ water film simultaneously. Impact angle is $45^{\circ}$ and normal impact velocity component is $3.5 \mathrm{~m} / \mathrm{s}$. 92 Figure 46. Top views of the two neighboring water droplets impact on water film in Figure 45. 92

Figure 47. Oblique impact of two neighboring water droplets impact on a 5- $\mu \mathrm{m}$ water film simultaneously. Impact angle is $45^{\circ}$ and normal impact velocity component is $53 \mathrm{~m} / \mathrm{s}$. . 94 Figure 48. Top views of the two neighboring water droplets impact on water film in Figure 47. 95

Figure 49. Oblique impact of two neighboring water droplets impact on a 5- $\mu \mathrm{m}$ water film asynchronously. Impact angle is $45^{\circ}$ and normal impact velocity component is $53 \mathrm{~m} / \mathrm{s} .96$ Figure 50. Top views of the two neighboring water droplets impact on water film in Figure 49. 97

Figure 51. Definition of water collection efficiency (Tan, 2004)............................... 100

Figure 52. Procedure of calculating water collection efficiency 105 
Figure 53. Simulation of oblique impact on dry solid surface, $D_{0}=100 \mu \mathrm{m}, V_{0}=12 \mathrm{~m} / \mathrm{s}$, $\alpha=37^{\circ}$, and $\mathrm{K}=186.6$. Secondary droplets are larger on the upstream side 107

Figure 54. Comparison of size distribution of the splashed secondary droplets between simulation and experiment. The time that experiment data was taken was unknown.... 108 Figure 55. Normalized distributions of secondary droplet diameter, velocity, and shooting angle for $\mathrm{K} \approx 1300$. 109

Figure 56. Droplet impingement on a thin water film. $\mathrm{D}_{0}=100 \mu \mathrm{m}, \mathrm{h}=5 \mu \mathrm{m}, \mathrm{V}_{0}=78 \mathrm{~m} / \mathrm{s}$ and $\mathrm{K}=667$

Figure 57. Three-dimensional simulation of droplets impact on a sphere. (a) Simulation setup. (b) zoomed-in view of droplets with three-level adaptive mesh refinement. (c) Boundary conditions: blue - inflow; red - symmetric; uncolored - outflow. 113 Figure 58. Water collection efficiency comparison between experiment and simulation. 114

Figure 59. Three-dimensional simulation setup of droplets impact on an MS(1)-317 airfoil. The zoomed-in view shows the two-level adaptive mesh refinement near the regions containing droplets. 116 Figure 60. Altered streamlines and droplet deformation due to interaction between droplets and airflow. 117

Figure 61. Water collection efficiencies of single-sized droplets.

Figure 62. Water collection efficiencies comparison of experiment, simulations, and LEWICE. 120 


\section{CHAPTER 1. GENERAL INTRODUCTION}

\subsection{Aircraft Icing Due to SLDs}

The growing concern about the danger imposed on aviation safety by the so called supercooled large droplets (SLDs) has intrigued research interests in droplet splashing. SLDs generally have a diameter larger than $50 \mu \mathrm{m}$, but they can be up to 100 times larger than typical cloud droplets that most icing encounters involve. SLDs exist in freezing drizzle and rain where they maintain liquid form even though the temperature is below freezing point. Due to their large size, SLDs have different characteristics than typical cloud droplets. First, as shown in Figure 1(a), SLDs are less entrained in the flow around wings, and are more likely to impinge further aft and sometimes onto areas not protected by de-icing devices to form clear and solid layer of glaze ice. In contrast, typical cloud droplets are smaller and usually follow the streamlines and strike the wing leading edge, which is protected by de-icing devices (Figure 1(b)). Second, unlike cloud droplets which freeze immediately after striking the wing surface, SLDs freeze partially and they can continue to spread onto the unprotected surface. Third, SLDs can splash into smaller secondary droplets which might re-impinge onto unprotected areas to form ice (Figure 1(a)). As a result, SLDs are recognized as a significant aviation hazard (Potapczuk et al., 1993; Bragg, 1996). Several fatal aviation accidents have been caused by SLDs and recent research has revealed that icing due to SLDs occurs more frequently than initially realized 
(Bragg, 1996; Wright \& Potapczuk, 1996; Rutkowski et al., 2003; Wright \& Potapczuk, 2004; Wright, 2005; Wright, 2006).
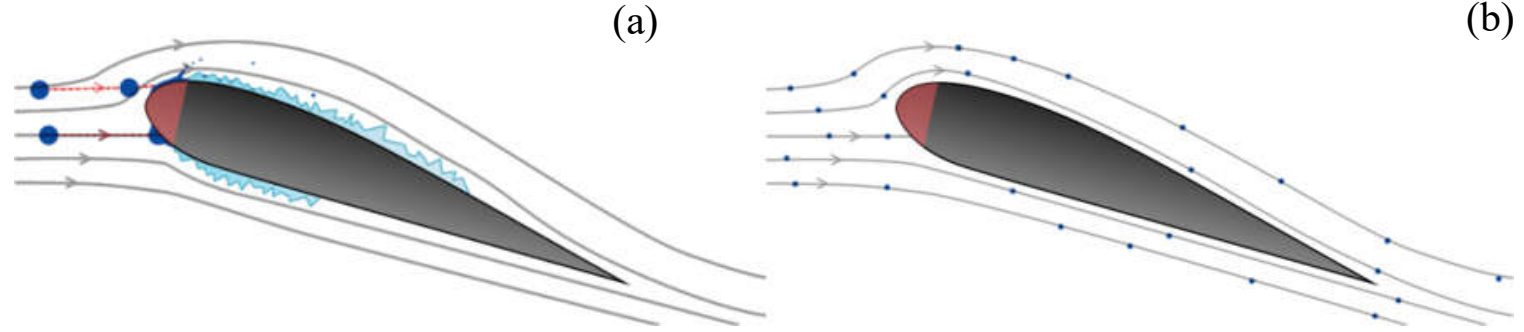

Figure 1. Characteristics of supercooled large droplet. (a) SLDs more likely hit the wing surface and move into areas not protected by de-icing devices to form ice; (b) typical cloud droplets usually follow the streamlines and likely hit the leading edge which is protected by de-icing devices (Protected area is shown in red).

Studies of SLD ice accretion process and its effect on aircraft performance have been performed by various researchers (Politovich, 1989; Potapczuk et al., 1993; Bragg, 1996; Bragg, 1996; Wright \& Potapczuk, 1996; Wright \& Potapczuk, 1996; Dunn et al., 1999; Lee \& Bragg, 1999; Isaac et al., 2001; Morency et al., 2001; Bond et al., 2003; Gent \& Moser, 2003; Gibou et al., 2003; Rutkowski et al., 2003; Wright \& Potapczuk, 2004; Wright, 2005; Wright, 2006). However, most existing simulation tools either ignore the droplet impingement or are based on simplified empirical splashing models. Myers and Hammond (Myers \& Hammond, 1999) use a 1-D control-volume approach to simulate ice growth in SLD conditions without considering actual droplet physics. Other simulation tools, instead of solving one set of governing equation, solve different sets of equations for 
airflow, droplet impingement, heat transfer, and phase change. FENSAP-ICE (Morency et al., 2001) solves four systems of PDEs in an interactive loop, including the compressible Navier-Stokes equations for airflow, an Eulerian method model for droplet impingement, a control volume equilibrium model for ice accretion and water runback, and a conjugate heat transfer model for phase change. LEWICE (Potapczuk et al., 1993; Wright, 2005; Wright, 2006) is based on the successive approach in which air flow, water droplet trajectories, heat transfer balance, and ice accretion are simulated by different software and these software loosely couple together to simulate the ice formation. The splashing models used in FENSAP-ICE and LEWICE are calibrated with low speed normal impact tests. These models in general do not give accurate prediction for high speed oblique impact. ONERA 2D icing suite (Villedieu et al., 2014) takes into account droplet impingement model, which is based on experimental results and simplified assumptions. ONERA 2D uses a Lagrangian trajectory solver and calculates the deposition probability of each droplet, then determines whether the droplet fully stick to solid surface or partial stick with the secondary droplets re-emitted into the air flow. In the SLD icing simulations of Silva et al. using CFD++ (Silva et al., 2014), they incorporated the effect of droplet-wall interaction into the dispersed phase momentum equation as a body force which is proposed by Honsek et al. (Honsek et al., 2008). These analysis tools focus on macro properties or model droplet impingement phenomenon empirically, which can lead to inaccuracies in predicting ice accretion. 


\subsection{Drop Impingement Dynamics}

Droplet splashing is considered as the most important aspect of the SLD ice accretion problem and studies show that ignoring droplet splashing leads to poor agreement with experimental results (Rutkowski et al., 2003; Wright, 2006). Extensive studies have been conducted to understand droplet impacts and splashing (Xu et al., 2005; Mani et al., 2010; Schroll et al., 2010; Latka et al., 2012; Mandre \& Brenner, 2012; Thoraval et al., 2012; Lian et al., 2014). Various splashing models and thresholds have been proposed (Lesser \& Field, 1983; Chandra \& Avedisian, 1991; Mundo et al., 1995; Yarin \& Weiss, 1995; Mundo et al., 1997; Pasandideh-Fard et al., 2001; Šikalo et al., 2005; Xu et al., 2005; Xu et al., 2007; Schroll et al., 2010; Kolinski et al., 2012; Latka et al., 2012; Mandre \& Brenner, 2012; Thoraval et al., 2012; Azimi et al., 2013; Lian et al., 2014). However, most studies focus on low speed impact rather than high speed impact that SLDs experience (Jung \& Myong; Wright \& Potapczuk, 2004). Very limited SLD studies have been conducted at inflight icing conditions (Bragg, 1996; Wright \& Potapczuk, 1996; Wright \& Potapczuk, 2004; Wright, 2006). These work typically focus on ice shapes and statistical properties such as impingement limit and water collection efficiency, but the fundamental physics of SLD impingement has not been considered (Wright \& Potapczuk, 1996; Wright, 2005; Wright, 2006).

Previous studies have established splashing threshold by systematically investigating the effects of liquid properties (density, viscosity, and surface tension), kinematic parameters (velocity and momentum), and surface conditions (roughness and wettability) (Mundo et al., 1995; Yarin \& Weiss, 1995; Bussmann et al., 1999; Rieber \& Frohn, 1999; Weiss \& Yarin, 1999; Rioboo et al., 2003; Yarin, 2006; Mongruel et al., 2009). The following group 
of dimensionless parameters has been identified to govern the outcome of droplet impingement on a smooth dry surface:

$$
\operatorname{Re}=\frac{\rho D V_{0}}{\mu} \quad W e=\frac{\rho D V_{0}^{2}}{\sigma} \quad O h=\frac{\mu}{(\rho \sigma D)^{1 / 2}}=\frac{W e^{1 / 2}}{\operatorname{Re}}
$$

where $\rho, \mu$, and $\sigma$ denote the liquid density, liquid viscosity, and gas-liquid surface tension, respectively, and $D$ and $V_{0}$ denote the droplet diameter and impact velocity normal to the surface, respectively. Surface roughness plays a critical role on splashing (Xu et al., 2005; Latka et al., 2012), but it will not be considered here because the wing surface is usually kept smooth to reduce skin friction drag (Lissaman, 1983; White, 2006).

Mundo et al. (Mundo et al., 1995) experimentally studied the splashing threshold and proposed a composite dimensionless parameter $K$, which is defined as follows:

$$
K=W e^{1 / 2} \cdot R e^{1 / 4}
$$

The splashing threshold of $K$ value is determined to be 57.7 from their experiments. For a SLD with a diameter of $50 \mu \mathrm{m}$ the splash threshold is exceeded when the normal impact velocity is larger than about $54 \mathrm{~m} / \mathrm{s}$, indicating splashing is a significant factor in large droplet regime (Wright \& Potapczuk, 2004). Other splashing models have also been proposed (Marengo \& Tropea; Stanton \& Rutland, 1998; Samenfink et al., 1999; Schmehl et al., 1999; Trujillo et al., 2000) but experimental studies show that these empirical models usually give accurate prediction for normal impacts but not for oblique impacts (Wright \& Potapczuk, 2004). Drop spreading, receding, and rebound usually can be predicted by analytical models (Chandra \& Avedisian, 1991; Xu et al., 1998; Kim \& Chun, 2001; Roisman et al., 2002; Mao et al., 2004). However, the splashing phenomenon is beyond 
the capability of these analytical models. Numerical studies have been performed to study droplet impacts. Blake et al. (Blake et al., 2014) simulated SLD impingement on a cooled substrate using ANSYS Fluent. However, no splashing was observed in any of their simulations even when the $K$ value is much higher than the splashing threshold of Mundo et al. Footte (Footte, 1975) studied the droplet impact on a thin film by solving the NavierStokes equations with the Marker-and-Cell-method (Brackbill et al.), and Bussmann et al. (Bussmann et al., 1999; Bussmann et al., 2000) studied 3D drop impacts on a thin film using the volume of fluid (VOF) method. They relied on specific perturbations to trigger splashing, but it is not clear whether these perturbations exist in the experiments.

There is another layer of complexity in the study of droplet impact and splashing. Ambient gas was not considered to affect the droplet impact initially (Bussmann et al., 2000; Yokoi, 2011). However, Xu et al. (Xu et al., 2005) and Latka et al. (Latka et al., 2012) found that the air plays a crucial role in determining the splashing behavior: a low pressure can effectively suppress the splashing. Splashing occurs when in atmospheric pressure, but is suppressed when the pressure is lowered to $13 \mathrm{kPa}$ even the $K$ value of 507 is much higher than the threshold of Mundo et al. (Mundo et al., 1997). Numerical simulation by Schroll et al. (Schroll et al., 2010) also demonstrated that the splashing can be suppressed when the ambient air effects are negligible. Lian et al. (Lian et al., 2014) numerically investigated the effect of air on droplet impact using the moment-of-fluid method. They observed that at low pressure the thin liquid sheet does not lift off even after it contacts the solid but at high pressure the thin sheet lifts off. Kolinski et al. (Kolinski et al., 2012) experimentally showed that during the droplet impact process a thin air film was trapped between the droplet and the solid surface. This air film serves to lubricate the droplet and makes the 
fluid to slide on the film at high velocities. All these studies underscore the importance of air in the droplet impact.

In addition to the ambient gas, the impact angle is also important to the outcome of splashing phenomenon (Liu et al., 2010). Mundo et al. (Mundo et al., 1995) experimentally showed that oblique impact has the same splash threshold when a normal velocity component is used. On the other hand, Bird et al. (Bird et al., 2009) demonstrated that the tangential velocity component plays a complicated role: it exacerbates splashing for lowspeed impact cases while it suppresses splashing for high-speed impact cases.

Droplet impact on thin liquid film is also an important phenomenon in aircraft in SLD conditions, as thin liquid films are created by the impacts of previous droplets. Experimental studies (Yarin \& Weiss, 1995; Cossali et al., 1997; Wang \& Chen, 2000; Rioboo et al., 2003) revealed the threshold for droplet splashing on thin liquid film. Yarin and Weiss proposed the threshold velocity for droplet splashing in a train:

$$
V_{0 S}=18\left(\frac{\sigma}{\rho}\right)^{1 / 4} v^{1 / 8} f^{3 / 8}
$$

where $f$ is the frequency of incoming droplets. For single droplet splashing, $f$ can be replaced with $V_{0} / D_{0}$. Their study showed that the droplet diameter has no effect on the splashing threshold and the liquid film thickness is less important. Cossali et al. proposed the threshold $K$ value for droplet splashing on thin liquid film:

$$
K_{S}=2100+5880\left(\frac{h}{D_{0}}\right)^{1.44} \quad \text { for } 0.1<\frac{h}{D_{0}}<1 \text { and } O h>0.007
$$


where the thickness of the liquid film, $h$, is considered. However, the equation is only valid for $h / D_{0}>0.1$. For $h / D_{0}<0.1$, experimental data from Wang and Chen and Rioboo et al. (Wang \& Chen, 2000; Rioboo et al., 2003) established the threshold $K_{S} \approx 400$.

\subsection{Thesis Organization}

Droplet splashing, the most important phenomenon in SLD, is still not fully understood (Rutkowski et al., 2003; Wright \& Potapczuk, 2004; Wright, 2006). In this study, droplet impingement and splashing on dry and wet surfaces are investigated using a multiphase flow solver.

Chapter 2 presents the numerical methods of the multiphase flow solver. The NavierStokes equations were solved using the variable density pressure projection method on a dynamic block structured adaptive grid. The moment of fluid method was used to reconstruct interfaces separating different phases. A dynamic contact angle model was used to define the boundary condition at the moving contact line.

Chapter 3 presents the numerical study of drop impingement and splashing on both dry and wet surfaces at impact velocities greater than $50 \mathrm{~m} / \mathrm{s}$ with consideration of the effect of surrounding air. The numerical method is validated by comparing with available experimental data. The low-speed and high-speed impacts on dry surface and thin liquid film are studied to highlight the different roles ambient air played in splashing.

Chapter 4 presents the numerical study of high-speed drop impact on thin liquid film with a focus on oblique impact. The numerical method is validated with experiment and 
theoretical solution. The normal and oblique impacts of water drops on water films of different impact angles and film thicknesses are investigated. The normal impacts of different film-drop density ratios are studied.

Chapter 5 presents the numerical study of the impact of multiple drops on a thin liquid layer. The numerical method is validated with experiments of normal impacts on liquid films. Two neighboring water droplets impacting on a water film at $45^{\circ}$ impact angle is simulated.

Chapter 6 presents an integrated Eulerian approach to study the water collection efficiency and impinge limit using the multiphase flow solver. Unlike most previous methods, the proposed approach considers the interplay between air and droplets. The droplet deformation and impingement on the wing surface and its subsequent behaviors are captured.

Chapter 7 concludes the dissertation. 


\section{CHAPTER 2. NUMERICAL METHODS}

The multiphase flow solver solves the three-dimensional Navier-Stokes equations using the variable density pressure projection algorithm (Kwatra et al., 2009) on a blockstructured adaptive mesh refinement (AMR) grid (Sussman et al., 1999). The solver employs state-of-the-art moment-of-fluid (MOF) method to represent the multiphase interfaces (Dyadechko \& Shashkov, 2005; Jemison et al., 2013; Lian et al., 2014). It employs dynamic contact angle models for droplet impact problems (Lian et al., 2014).

\subsection{Governing Equations}

The continuity, momentum, and material indicator equations for incompressible, immiscible, multiphase flows are written as follows:

$$
\begin{gathered}
\nabla \cdot \mathbf{U}=0 \\
\rho(\mathbf{H})\left(\frac{\partial \mathbf{U}}{\partial t}+\mathbf{U} \cdot \nabla \mathbf{U}\right)=-\nabla p+\nabla \cdot \tau+F_{\text {tension }} \\
\frac{\partial \mathbf{H}}{\partial t}+\nabla \cdot(\mathbf{U H})=0
\end{gathered}
$$

where $\mathbf{U}=(u, v, w)$ is the velocity vector, $t$ is the time, $p$ is the pressure, and $\mathbf{H}(\mathbf{x}, t)=\left(H_{1}, H_{2}, \ldots, H_{M}\right)$ is the material indicator vector function. The material indicator function is defined as follows: 


$$
H_{m}(\mathbf{x}, t)= \begin{cases}1 & \mathbf{x} \in \text { material } \mathrm{m} \\ 0 & \text { otherwise }\end{cases}
$$

$\rho(\mathbf{H}) \equiv \sum_{m=1}^{M} \rho_{m} H_{m}$ is the density, and $\tau$ is the shear stress tensor,

$$
\tau=\mu(\mathbf{H})\left(\nabla \mathbf{U}+(\nabla \mathbf{U})^{T}\right)
$$

here $\mu(\mathbf{H}) \equiv \sum_{m=1}^{M} \mu_{m} H_{m}$ is the viscosity, and the stress at the material interface will have the following jump condition due to the surface tension

$$
[(-p I+\tau) \cdot \mathbf{n}]=\sigma \kappa \mathbf{n}
$$

where $\sigma$ is the surface tension coefficient, $\kappa$ and $\mathbf{n}$ are the curvature and unit normal of the interface respectively. For two-phase flow the surface tension term, $\boldsymbol{F}_{\text {tension, }}$ is

$$
\mathbf{F}_{\text {tension }}=\sigma_{1,2}\left(\nabla \cdot \mathbf{n}_{1}\right) \nabla H_{1}
$$

and the interface unit normal is

$$
\mathbf{n}_{1}=\frac{\nabla H_{1}}{\left|\nabla H_{1}\right|}
$$

For multiphase flow ( $M \geq 3$ ), the exact form of the surface tension terms can be found in (Li et al., 2015).

\subsection{Moment-of-fluid interface reconstruction}

The MOF method (Dyadechko \& Shashkov, 2005; Ahn \& Shashkov, 2007; Dyadechko \& Shashkov, 2008; Ahn \& Shashkov, 2009; Jemison et al., 2012) has been developed for computing the motion of deforming boundary problems. In the MOF method, we represent 
the material $m$ distribution using its volume fraction and centroid. In each computational cell, the volume fraction is

$$
F_{m}=\frac{\Omega_{i, m}}{\Omega_{i}}=\frac{1}{\Delta x \Delta y \Delta z} \int_{x_{i-1 / 2}}^{x_{i+1 / 2}} \int_{y_{j-1 / 2}}^{y_{j+1 / 2}} \int_{z_{k-1 / 2}}^{z_{k+1 / 2}} H_{m}(\mathbf{x}) d z d y d x
$$

where $\Omega_{i}$ and $\Omega_{i, m}$ are the volume of cell $(i, j, k)$ and volume of material $m$ in the cell. The centroid of the material $m$ in the cell is

$$
\mathbf{x}_{m}=\frac{\int_{x_{i-1 / 2}}^{x_{i+1 / 2}} \int_{y_{j-1 / 2}}^{y_{j+1 / 2}} \int_{z_{k-1 / 2}}^{z_{k+1 / 2}} H_{m}(\mathbf{x}) \mathbf{x} d z d y d x}{\int_{x_{i-1 / 2}}^{x_{i+1 / 2}} \int_{y_{j-1 / 2}}^{y_{j+1 / 2}} \int_{z_{k-1 / 2}}^{z_{k+1 / 2}} H_{m}(\mathbf{x}) d z d y d x}
$$

Without phase change, each material will maintain its own state along the streamline. Therefore, we can use Eq. (7) to track the change of the material indicator.

In each computational cell, each interface between different phases is represented by a plane in $3 \mathrm{D}$ or a line in $2 \mathrm{D}$, which is called the piecewise linear interface calculation (PLIC). For 2D the interface can be represented by a straight line as shown in Figure 2 using the following equation:

$$
\mathbf{n} \cdot\left(\mathbf{x}-\mathbf{x}_{i, j}\right)+b=0
$$

where $\mathbf{n}$ is the unit normal vector, $\mathbf{x}_{i, j}$ is the cell center, and $b$ the distance from the cell center to the line. 


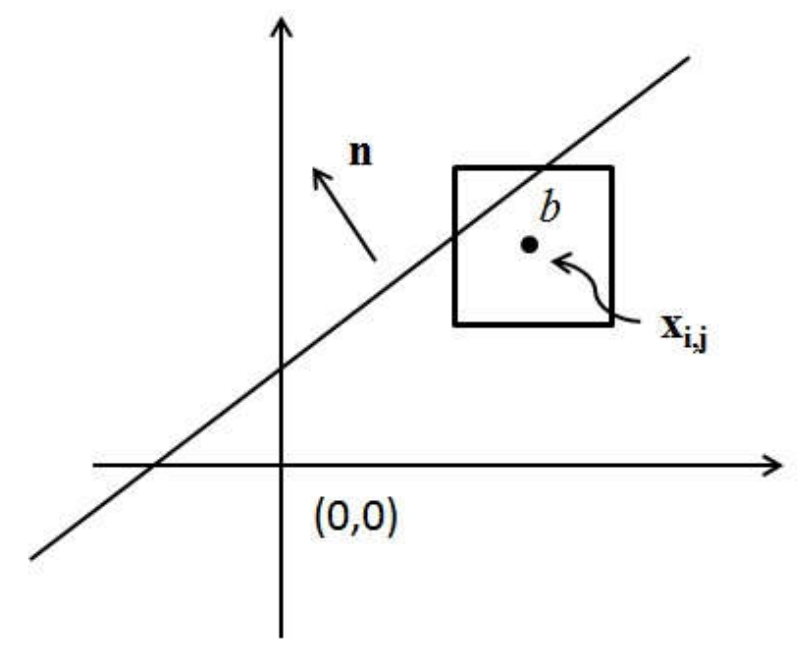

Figure 2. The interface can be represented by a straight line in $2 \mathrm{D}$ case. The square represents a computational cell.

The MOF method, which can be considered as a generalization of the volume of fluid (VOF) method, uses both the volume fraction function, $F_{m}=F_{m}(\mathbf{n}, b)$, and the corresponding material centroid, $\mathbf{x}_{m}^{c}=\mathbf{x}_{m}^{c}(\mathbf{n}, b)$, to construct the interface. Given a reference volume fraction function, $F_{r e f, m}$, and a reference centroid, $\mathbf{x}_{r e f, m}^{c}$, in one computational cell, the MOF interface reconstruction requires that the actual volume fraction function $\left(F_{a c t, m}=F_{a c t, m}(\mathbf{n}, b)\right)$ matches the reference volume fraction function ( $\left.F_{r e f, m}\right)$ exactly and the actual centroid $\left(\mathbf{x}_{a c t, m}^{c}=\mathbf{x}_{a c t, m}^{c}(\mathbf{n}, b)\right)$ is the best possible approximation to the reference centroid $\left(\mathbf{x}_{r e f, m}^{c}\right)$ as illustrated in Figure 3. 

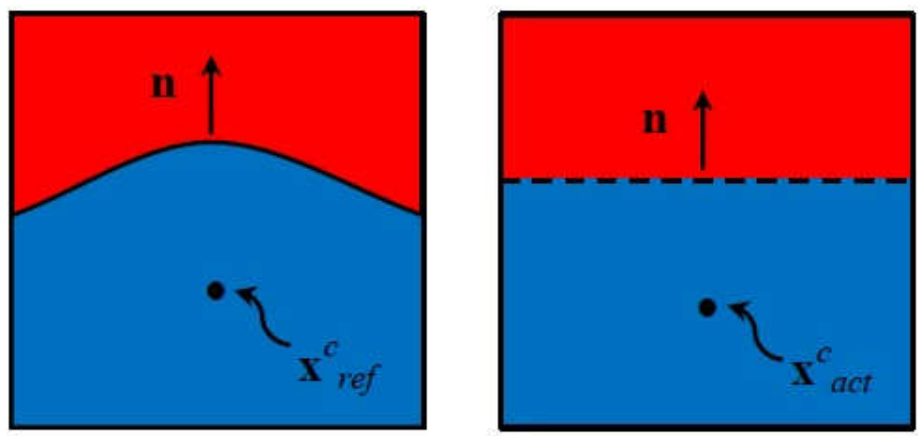

Figure 3. MOF interface reconstruction. The solid curved line represents the true interface and the dashed straight line represents the reconstructed interface.

This procedure can be achieved by minimizing the following function

$$
E_{M O F}=\left\|\mathbf{x}_{r e f, m}^{c}-\mathbf{x}_{a c t, m}^{c}(\mathbf{n}, b)\right\|^{2}
$$

with the following constraint

$$
\left|F_{r e f, m}-F_{a c t, m}(\mathbf{n}, b)\right|=0
$$

To find the normal vector $\mathbf{n}$ and intercept $b$, we first parameterize $\mathbf{n}$ as follows:

$$
\mathbf{n}=\left(\begin{array}{c}
\sin (\Phi) \cos (\Theta) \\
\sin (\Phi) \sin (\Theta) \\
\cos (\Phi)
\end{array}\right)
$$

then the minimization problem of Eq. $(16)$ becomes solving $\left(\Phi^{*}, \Theta^{*}\right)$ so that

$$
E_{M o F}\left(\Phi^{*}, \Theta^{*}\right)=\min \left\|\mathbf{x}_{r e f, m}^{c}-\mathbf{x}_{a c t}^{c}(\Phi, \Theta)\right\|_{2}
$$

Eq. (19) is solved numerically by the Gauss-Newton algorithm and the detailed step-bystep procedure is described by Jemison et al. (Jemison et al., 2014). 
As one can see from the previous discussion, unlike the VOF method, the MOF interface reconstruction method only uses information from the computational cell under consideration. This characteristic makes the MOF method more suitable for multiphase problems with sharp corners. Furthermore, it makes the MOF method more suitable for block structured adaptive mesh refinement (Jemison et al., 2012).

\subsection{Moment-of-fluid interface advection}

Once the interface is constructed, the next step is to advect the interface to the next time instant. We use the direction splitting (Strang, 1968; Jemison et al., 2012) technique which allows us to integrate the interface position sequentially. Here we will illustrate the interface integration process only in the $x$ direction.

As depicted in Figure 4, for a given computational cell $i$ occupying region $\Omega_{i}$, we first trace backward in time to find its previous position $\Omega_{i, d}$, which is called the departure region (the dashed region) and is defined as

$$
\Omega_{i}^{D}=\left[x_{i-1 / 2}-u_{i-1 / 2} \Delta t, x_{i+1 / 2}-u_{i+1 / 2} \Delta t\right]
$$

$u_{i-1 / 2}$ and $u_{i+1 / 2}$ are horizontal velocities on the cell interface. The departure region will advect to the target region (cell $i$ itself)

$$
\Omega_{i}^{T}=\Omega_{i}=\left[x_{i-1 / 2}, x_{i+1 / 2}\right]
$$

We define a mapping function $T_{\mathrm{i}}$ so that

$$
T_{i}: \Omega_{i}^{D} \rightarrow \Omega_{i}^{T}
$$


For $T_{i}$ in Eq. (21) the following linear mapping function is used

$$
x^{T}=\alpha x+\beta=\frac{\Delta x\left(x-\left(x_{i-1 / 2}-u_{i-1 / 2} \Delta t\right)\right)}{\left(x_{i+1 / 2}-u_{i+1 / 2} \Delta t\right)-\left(x_{i-1 / 2}-u_{i-1 / 2} \Delta t\right)}+x_{i-1 / 2}
$$

If $\left|\Omega_{i}^{D}\right|>\left|\Omega_{i}^{T}\right|$, then the material in the departure region undergoes compression; otherwise, the material in the departure region undergoes expansion.

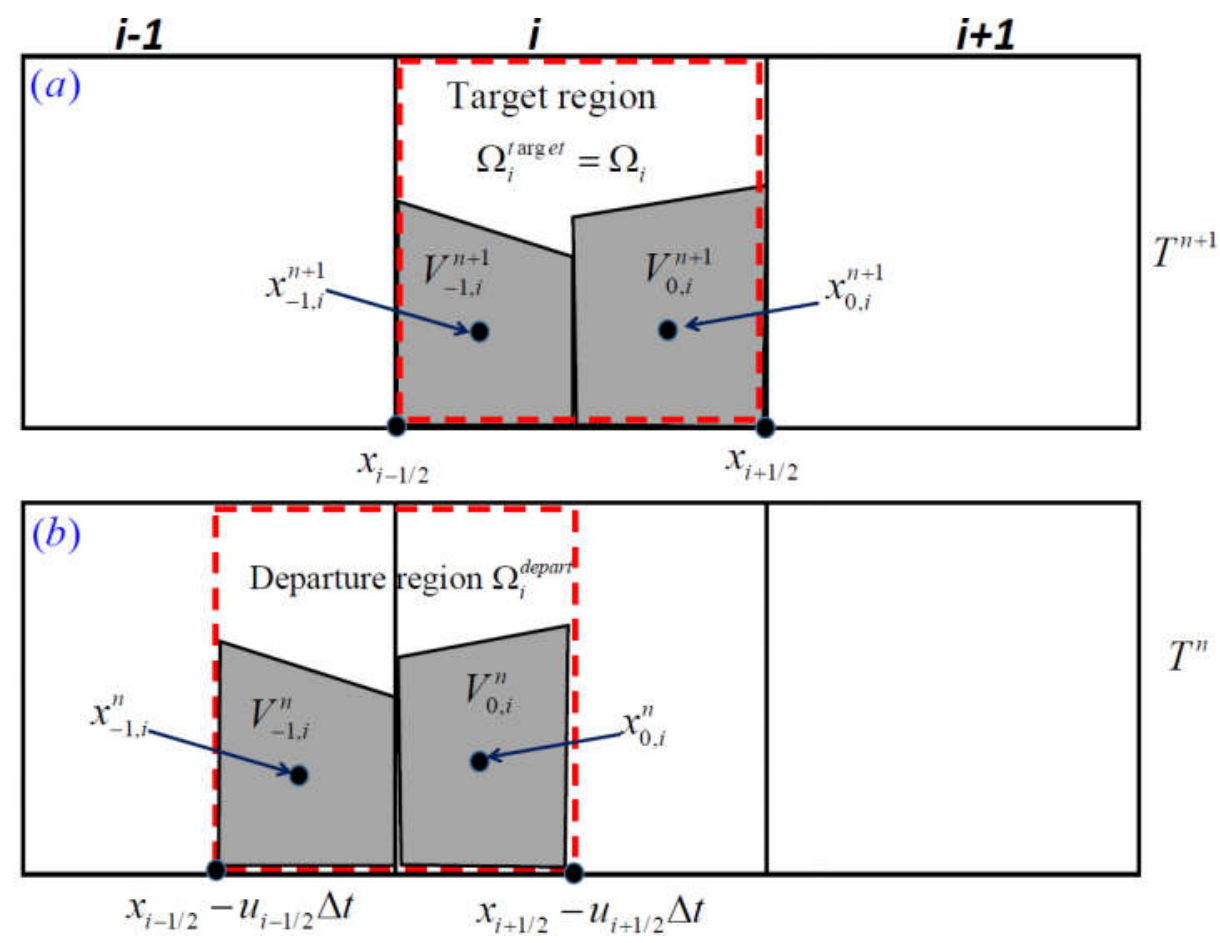

Figure 4. Illustration of backward tracking process. The dashed region in (b) represents the departure region of cell $i, \Omega_{i}{ }^{D}$; the dashed square in (a), $\Omega_{i}$, is the target region. The shaded region in (b), $\Omega_{m, i}^{D}=V_{-1, i}^{n} \cup V_{0, i}^{n}$, is the material $m$, in the departure region. The shaded region in (a), $\Omega_{m, i}^{T}=V_{-1, i}^{n+1} \cup V_{0, i}^{n+1}$, is the material $m$, in the target region. 
Now we illustrate how to advect the interface using backward projection process. For that purpose we introduce new notations, $V_{-1, i}^{n}$, and $V_{0, i}^{n}$, which are the interactions of material $m$ in underlying grids with the departure region, i.e., $V_{-1, i}^{n} \equiv \Omega_{m, i-1}^{n} \cap \Omega_{i}^{D}$, and $V_{0, i}^{n} \equiv \Omega_{m, i}^{n} \cap \Omega_{i}^{D}$. Here $\Omega_{m, i-1}^{n}$ and $\Omega_{m, i}^{n}$ represent material $m$ in cell $i-1$ and $i$ respectively at time instant $t^{n}$. At time $t^{n+1}$ region $V_{-1, i}^{n}$ and $V_{0, i}^{n}$ will be advected into the target region to become $V_{-1, i}^{n+1}$ and $V_{0, i}^{n+1}$. As shown in Figure 4, the volume fraction of material $m$ in the target region is then

$$
F_{m, i}^{n+1}=\frac{\left|V_{-1, i}^{n+1}\right|+\left|V_{0, i}^{n+1}\right|}{\left|\Omega_{i}^{T}\right|}=\frac{\left|T_{i}\left(V_{-1, i}^{n}\right)\right|+\left|T_{i}\left(V_{0, i}^{n}\right)\right|}{\left|\Omega_{i}^{T}\right|}=\frac{\left|T_{i}\left(\Omega_{m, i-1}^{n} \cap \Omega_{i}^{D}\right)\right|+\left|T_{i}\left(\Omega_{m, i}^{n} \cap \Omega_{i}^{D}\right)\right|}{\left|\Omega_{i}^{T}\right|}
$$

and the centroid of material $m$ in the target cell $\Omega_{\uparrow}$ is

$$
\mathbf{x}_{m, i}^{n+1}=\frac{\int_{V_{-1, i}^{n+i}} \mathbf{x} d \mathbf{x}+\int_{V_{0, i}^{n+i}} \mathbf{x} d \mathbf{x}}{F_{m, i}^{n+1}\left|\Omega_{i}^{T}\right|}=\frac{\int_{T_{i}\left(\Omega_{n, i,-1}^{n} \cap \Omega_{i}^{D}\right)} \mathbf{x} d \mathbf{x}+\int_{T_{i}\left(\Omega_{n, i}^{n} \cap \Omega_{i}^{D}\right)} \mathbf{x} d \mathbf{x}}{F_{m, i}^{n+1}\left|\Omega_{i}^{T}\right|}
$$

In general, the volume fraction function and the centroid of material $m$ in the next time step are

$$
\begin{aligned}
F_{m, i}^{n+1} & =\frac{\sum_{i^{\prime}=-1}^{1}\left|T_{i}\left(\Omega_{m, i+i^{\prime}}^{n} \cap \Omega_{i}^{D}\right)\right|}{\left|\Omega_{i}^{T}\right|} \\
\mathbf{x}_{m, i}^{n+1} & =\frac{\sum_{i^{\prime}=-1}^{1} \int_{T_{i}\left(\Omega_{m, i+i}^{n} \cap \Omega_{i}^{D}\right)} \mathbf{x} d \mathbf{x}}{F_{m}^{n+1}\left|\Omega_{i}^{T}\right|}
\end{aligned}
$$

Note that Eq. (26) is the volume fraction only from the contribution of the $x$ direction. The above process should be repeated to advect the interface in $y$ and $z$ directions in order to 
update the volume fraction and centroid position. Typically, the "Strang splitting" method is used to advect the interface successively in the $x, y$, and $z$ directions, and then the order is reserved to be $z, y$, and $x$ directions. More details can be found in (Jemison et al., 2012).

\subsection{Fluid solver}

For incompressible flow simulation we apply a time splitting approach as discussed in Kwatra et al. (Kwatra et al., 2009). The approach essentially consists of two steps. In the first step, advection terms are calculated to obtain an intermediate velocity and density fields; in the second step, these fields are corrected by the pressure field. The advected velocity field is calculated in a similar way as the material volume fraction and centroid,

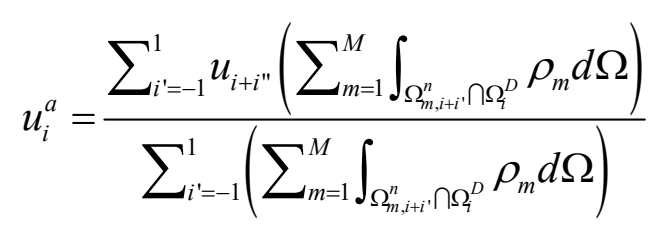

Once the advected velocity, $u^{a}$, is found, the velocity at $t^{n+1}$ is updated as

$$
u^{n+1}=u^{a}-\Delta t \frac{\nabla p^{n+1}}{\rho^{n+1}}
$$

Taking the divergence of Eq. (29) and enforcing incompressibility at $t^{n+1}\left(\nabla \cdot u^{n+1}=0\right)$ gives

$$
\frac{1}{\Delta t} \nabla \cdot u^{a}=\nabla \cdot\left(\frac{\nabla p^{n+1}}{\rho^{n+1}}\right)
$$

For the 1D problem, as an illustration, Eq. (30) is discretized in a control volume style 


$$
\left(\frac{\left(\frac{p_{i+1}^{n+1}-p_{i}^{n+1}}{\rho_{i+1 / 2}^{n+1} \Delta x}\right)-\left(\frac{p_{i}^{n+1}-p_{i-1}^{n+1}}{\rho_{i-1 / 2}^{n+1} \Delta x}\right)}{\Delta x}\right)=\frac{1}{\Delta t} \frac{u_{i+1 / 2}^{a}-u_{i-1 / 2}^{a}}{\Delta x}
$$

here the density and velocity are taken at cell faces. To facilitate the calculation of the cell face values, we divide cell $i$ into two control volumes, $\Omega_{i, L}$ and $\Omega_{i, R}$. The densities averaged over these two control volumes are

$$
\begin{gathered}
\rho_{i, R}=\frac{1}{\left|\Omega_{i, R}\right|} \sum_{m=1}^{M}\left(\int_{\Omega_{m, i} \cap \Omega_{i, R}} \rho_{m} d \Omega\right) \\
\rho_{i+1, L}=\frac{1}{\left|\Omega_{i+1, L}\right|} \sum_{m=1}^{M}\left(\int_{\Omega_{m, i+1} \cap \Omega_{i+1, L}} \rho_{m} d \Omega\right)
\end{gathered}
$$

The density on the cell face $\rho_{i+1 / 2}$ is defined as

$$
\rho_{i+1 / 2}=\frac{\rho_{i, R}\left|\Omega_{i, R}\right|+\rho_{i+1, L}\left|\Omega_{i+1, L}\right|}{\left|\Omega_{i+1 / 2}\right|}
$$

where $\Omega_{t+1 / 2}$ is the face control volume defined as follows:

$$
\Omega_{i+1 / 2}=\Omega_{i, R} \cup \Omega_{i+1, L}
$$

The advective velocity on the cell face is defined as a mass-weighted interpolation of the cell centered advective velocity

$$
u_{i+1 / 2}^{a}=\frac{u_{i}^{a} \rho_{i, R}\left|\Omega_{i, R}\right|+u_{i+1}^{a} \rho_{i+1, L}\left|\Omega_{i+1, L}\right|}{\rho_{i+1 / 2}\left|\Omega_{i+1 / 2}\right|}
$$

and such an interpolation maintains the momentum conservation. 
Solving Eq. (31) yields the new pressure field $p^{n+1}$. The pressure field is then used to update the velocity using Eq. (29) as follows:

$$
\frac{\left(u_{i}^{n+1}-u_{i}^{a}\right)}{\Delta t}=-\frac{p_{i+1 / 2}^{n+1}-p_{i-1 / 2}^{n+1}}{\Delta x \rho_{i}^{n+1}}
$$

here $p_{i+1 / 2}^{n+1}$ and $p_{i-1 / 2}^{n+1}$ are the pressures at cell faces. Following Kwatra et al. (Kwatra et al., 2009) we define the momentum equation in control volume, $\Omega_{\uparrow, R}$ and $\Omega_{\uparrow+1, L}$

$$
\begin{gathered}
\frac{D u_{i, R}}{D t}=\frac{u_{i, R}^{n+1}-u_{i, R}^{a}}{\Delta t}=-\frac{p_{i+1 / 2}^{n+1}-p_{i}^{n+1}}{\rho_{i, R}^{n+1} \Delta x / 2} \\
\frac{D u_{i+1, L}}{D t}=\frac{u_{i+1, L}^{n+1}-u_{i+1, L}^{a}}{\Delta t}=-\frac{p_{i+1}^{n+1}-p_{i+1 / 2}^{n+1}}{\rho_{i+1, L}^{n+1} \Delta x / 2}
\end{gathered}
$$

Since at the cell interface $i+1 / 2$ fluid velocity maintains the same, we have the constraint that $\frac{D u_{i+1 / 2, R}}{D t}=\frac{D u_{i+1, L}}{D t}$. Using this constraint and Eq. (38) and (39), we have

$$
\frac{p_{i+1 / 2}^{n+1}-p_{i}^{n+1}}{\rho_{i, R}^{n+1} \Delta x / 2}=\frac{p_{i+1}^{n+1}-p_{i+1 / 2}^{n+1}}{\rho_{i+1, L}^{n+1} \Delta x / 2}
$$

The pressure at the cell face can be found

$$
p_{i+1 / 2}^{n+1}=\frac{\rho_{i, R}^{n+1} p_{i+1}^{n+1}+\rho_{i+1, L}^{n+1} p_{i}^{n+1}}{\rho_{i, R}^{n+1}+\rho_{i+1, L}^{n+1}}
$$

For viscous flows, we use the following equation to calculate the intermediate velocity

$$
\frac{\rho^{n+1}\left(u^{*}-u^{a}\right)}{\Delta t}=\nabla \cdot \tau\left(u^{a}\right)
$$


where $u^{*}$ is intermediate velocity field and $\tau$ is the stress tensor. We still use Eq. (31) to calculate the updated pressure field $p^{n+1}$ but we will replace $u^{a}$ with $u^{*}$ in Eq. (37) to correct the velocity field $u^{n+1}$. In the discretization of Eq. (42) we define the viscosity at cell interface as follows:

$$
\mu_{i+1 / 2}=\frac{\left|\Omega_{i+1 / 2}\right|}{\sum_{m=1}^{M} \frac{1}{\mu_{m}}\left(\left|\Omega_{i}^{m} \cap \Omega_{i, R}\right|+\left|\Omega_{i+1}^{m} \cap \Omega_{i+1, L}\right|\right)}
$$

We use the approach of Jemison (Jemison et al., 2014) to treat the surface tension. The ghost fluid method is used to discretize the surface tension force and curvature is calculated using the height function.

The Navier-Stokes equations for incompressible two-phase flows are solved using the variable density pressure projection algorithm (Kwatra et al., 2009) on the block structured adaptive mesh refinement (AMR) grids (Sussman et al., 1999). The grid adaption is based on the triple point region and the curvature of the interface. As shown in Figure 5, the grid refinement is performed near regions where curvature is higher than predefine value (here we use 0.1). The adaptive mesh refinement method ensures fine grid is only used in the regions of interests, which maintains the accuracy of the solver at reasonable computational cost. Another example of adaptive mesh refinement in a three-dimensional simulation case is shown in Figure 6. 


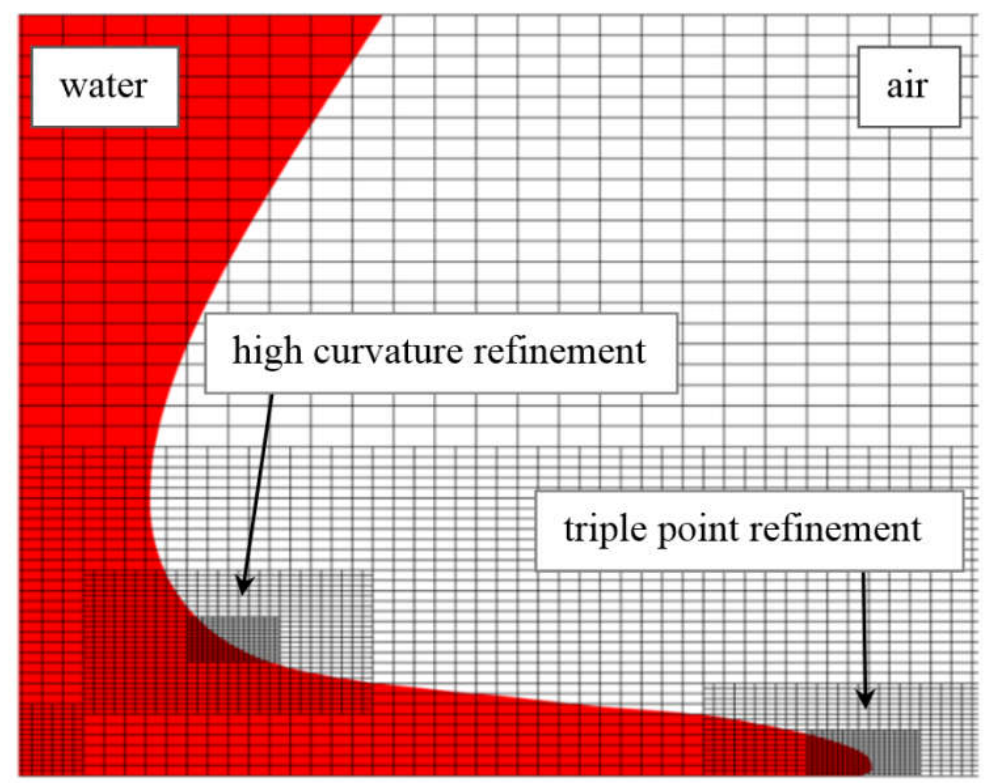

Figure 5. Three-level adaptive mesh refinement based on triple point and curvature of the interface in a two-dimensional simulation.

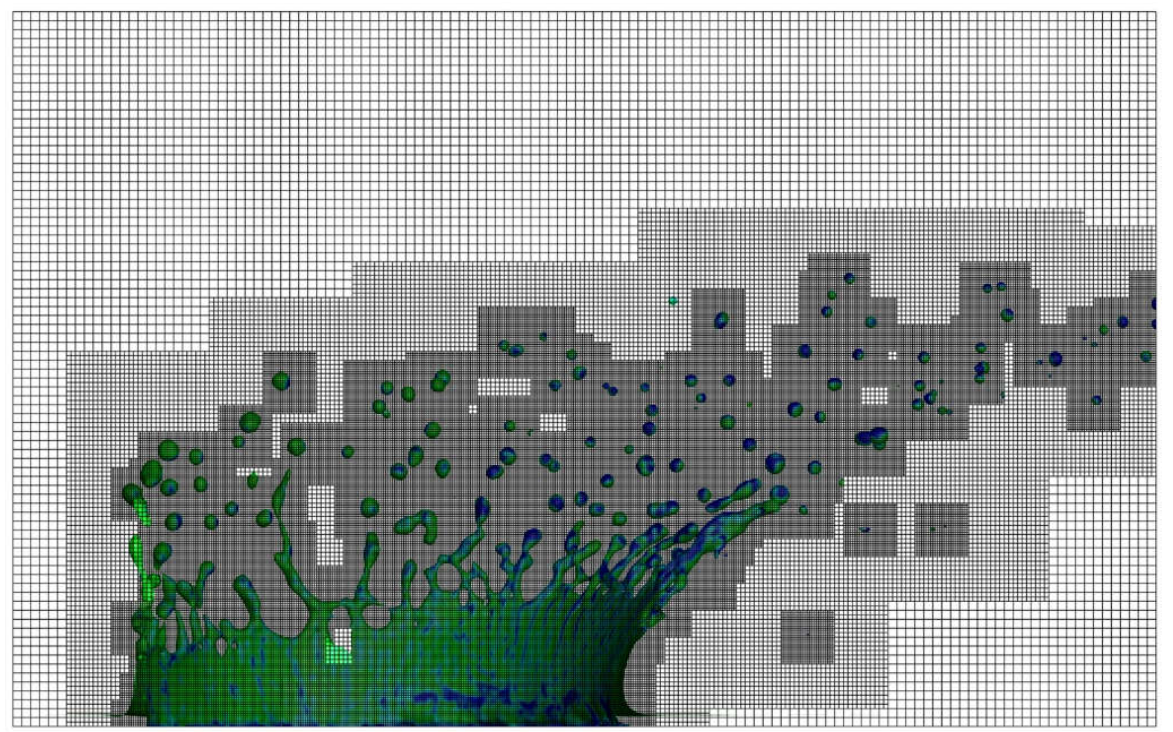

Figure 6. Two-level adaptive mesh refinement in a three-dimensional simulation. 


\subsection{Dynamic contact angle models}

In the simulations of droplet impacts the contact angle is required as a boundary condition at the contact line. Previous studies showed that specifying the correct contact angle is critical for the accuracy of numerical simulations (Šikalo et al., 2005; Criscione et al., 2011).

Three dynamic contact angle models, the model of Jiang et al. (Jiang et al., 1979), the model of Kistler (Kistler, 1993), and the model of Yokoi et al. (Yokoi et al., 2009), were tested. These models are calibrated based on different experiments, therefore, as shown in Figure 7, they show different dependency on the Capillary number. The models of Jiang and Kistler are derived from experimental measurement by Hoffman (Hoffman, 1975) who studied 5 fluids and measured the advancing contact angle during steady-state displacement of liquid-air phase interfaces through a glass capillary tube. The capillary number range is between $4 \times 10^{-5}$ and 36 . Jiang's model is a least-square fit of Hoffman's data and Kistler's model is only based on Hoffman's data points of completely wetting systems with high liquid viscosity and is fit at large $C a$ number. Yokoi et al. [61] developed four models based on their experiment of a $2.28 \mathrm{~mm}$ water droplet impacting onto a silicon wafer at 1 $\mathrm{m} / \mathrm{s}$. In Figure 7, the equilibrium contact angle, $\theta_{e}$, is $87.4^{\circ}$, and the equilibrium contact angle is specified by the surface tension between the gas, liquid and solid phases in code implementation. $\sigma_{l g}, \sigma_{l s}, \sigma_{s g}$ are used to denote the surface tension between liquid and gas, liquid and solid, and solid and gas, respectively. Since the models of Jiang et al. and Kistler are only valid for advancing contact angles, a constant receding contact angle from experimental measurement is implemented in the code for receding motion. Yokoi's model 
I is plotted in the figure and the maximum advancing contact angle and minimum receding contact angle are defined as $114^{\circ}$ and $52^{\circ}$ respectively.

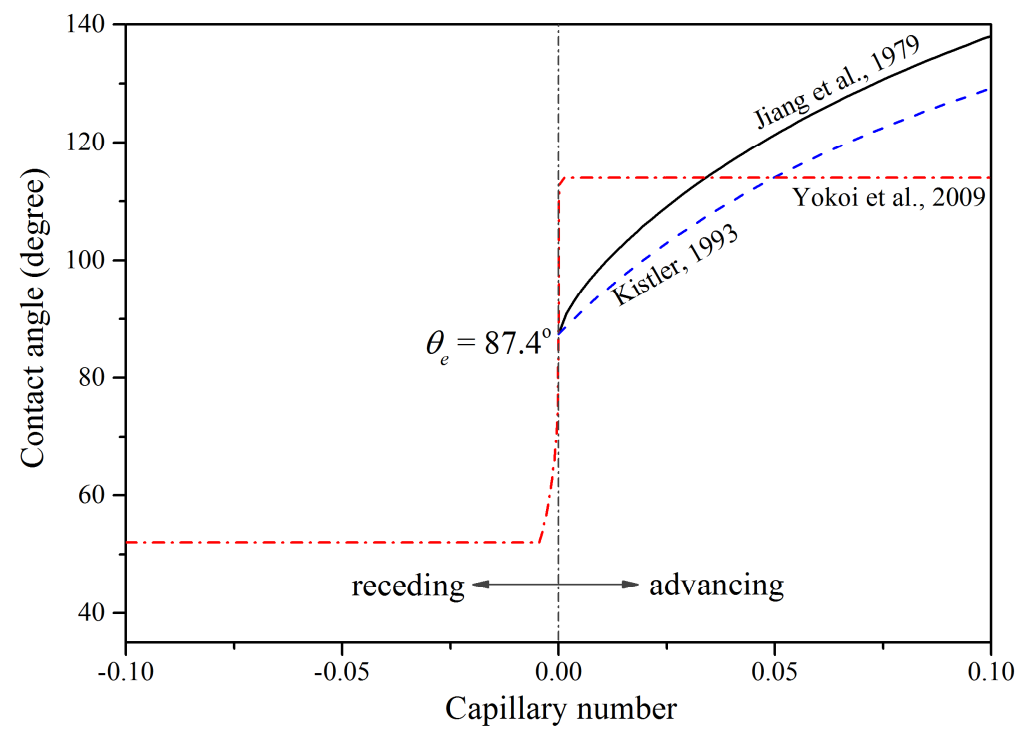

Figure 7. Comparison of three dynamic contact angle models vs. the Capillary number. 


\section{CHAPTER 3. DROP IMPACT ON DRY AND WET SURFACES WITH CONSIDERATION OF SURROUNDING AIR ${ }^{1}$}

\subsection{Introduction}

Droplet impingement on dry and wet surfaces is a ubiquitous phenomenon and has been of research interest for more than a century (Worthington, 1908). It plays an important role in areas such as industrial applications (e.g. spry cooling, spry coating, inkjet printing, and metal deposition in manufacturing processes), agricultural applications (e.g. pesticide spraying), forensic science (e.g. bloodstain pattern analysis) and fire suppression (e.g. sprinkler system). Aviation hazards due to ice accretion also involve droplet impingement. Ice accretion due to small cloud-sized droplets is well understood and can be prevented. However, supercooled large droplets with diameter larger than $50 \mu \mathrm{m}$ exhibit different characteristics during impingement and are recognized as a significant aviation hazard (Potapczuk et al., 1993; Bragg, 1996; Wright \& Potapczuk, 1996; Rutkowski et al., 2003; Wright \& Potapczuk, 2004; Wright, 2005; Wright, 2006). Among the physics phenomena

${ }^{1}$ This chapter appeared in the following publication:

Yisen Guo, Yongsheng Lian, and Mark Sussman, "Investigation of drop impact on dry and wet surfaces with consideration of surrounding air," Phys. Fluids 28, 073303 (2016). (AIP Publishing permits authors to include their published articles in a thesis or dissertation. Formal permission from AIP Publishing is not needed. (https://publishing.aip.org/authors/copyright-reuse)) 
involved, droplet splashing is considered as the most important aspect of the large droplet impact and studies have shown that ignoring droplet splashing leads to poor agreement with experimental results (Rutkowski et al., 2003; Wright, 2006). Extensive studies have been conducted to understand droplet impact and splashing (Xu et al., 2005; Mani et al., 2010; Schroll et al., 2010; Latka et al., 2012; Mandre \& Brenner, 2012; Thoraval et al., 2012; Lian et al., 2014). Various splashing models and thresholds have been proposed (Lesser \& Field, 1983; Chandra \& Avedisian, 1991; Mundo et al., 1995; Yarin \& Weiss, 1995; Mundo et al., 1997; Pasandideh-Fard et al., 2001; Šikalo et al., 2005; Xu et al., 2005; Xu et al., 2007; Schroll et al., 2010; Kolinski et al., 2012; Latka et al., 2012; Mandre \& Brenner, 2012; Thoraval et al., 2012; Azimi et al., 2013; Lian et al., 2014). Nevertheless, most studies focus on low-speed impact rather than high speed impact that most flights experience (Jung \& Myong; Wright \& Potapczuk, 2004). Very limited studies have been conducted at in-flight conditions for large droplets (Bragg, 1996; Wright \& Potapczuk, 1996; Wright \& Potapczuk, 2004; Wright, 2006). These work typically focus on statistical properties such as impingement limit and water collection efficiency, but the fundamental physics of splashing has not been sufficiently investigated (Wright \& Potapczuk, 1996; Wright, 2005; Wright, 2006).

Mundo et al. (Mundo et al., 1995) experimentally showed that there are two possible outcomes from the impingement of droplet on a flat dry surface: less energetic droplets deposit on the surface and form a liquid film, while energetic droplets splash to form secondary droplets. They characterized the transition from deposition to splashing using a dimensionless parameter $K\left(=O h \cdot R e^{1.25}\right): K>57.7$ indicating splashing. Here the Reynolds number and the Ohnesorge number are defined based on droplet properties 


$$
\operatorname{Re}=\frac{\rho D V_{0}}{\mu}, \quad O h=\frac{\mu}{(\rho \sigma D)^{1 / 2}}
$$

where $\rho, \mu$, and $\sigma$ are the droplet density, viscosity, and surface tension, respectively, and $D$ and $V_{0}$ are the drop diameter and terminal impact velocity, respectively. Rioboo et al. (Rioboo et al., 2001) revealed six outcomes of droplet impact on a dry surface, ranging from deposition to complete rebound. They also pointed out that surface wettability and roughness played important roles in the outcomes.

For drop spreading, receding, and rebound, analytical models give fairly accurate predictions (Chandra \& Avedisian, 1991; Kim \& Chun, 2001; Roisman et al., 2002; Mao et al., 2004). Numerical studies are also able to duplicate the experimental results. Footte (Footte, 1975) studied the droplet rebound by solving the Navier-Stokes equations with the Marker-and-Cell method. Pasandideh-Fard et al. (Pasandideh-Fard et al., 2001) used the volume of fluid method to investigate viscous drop spreading on dry and partially wettable surfaces: both constant and variable contact angles were applied as boundary condition at the contact line and the variable contact angle gave a better prediction of the drop spreading diameter. Bussmann et al. (Bussmann et al., 1999) simulated droplet deformation of oblique impacts on dry surfaces and the results showed good agreement with their experiments.

For droplet splashing on dry surfaces, analytical models cannot give accurate predictions. Studies have attempted to investigate droplet splashing mechanism at low-speed regions (Jayaratne \& Mason, 1964; Stow \& Hadfield, 1981; Fukai et al., 1995; Zhao et al., 1998; Bussmann et al., 1999; Sussman et al., 1999; Weiss \& Yarin, 1999; Bussmann et al., 2000; Zheng \& Zhang, 2000; Šikalo et al., 2005; Tanguy \& Berlemont, 2005). The most prevalent 
splash mechanism invokes the liquid compressibility and the geometric singularity due to the parabolic interface shape (Lesser \& Field, 1983; Haller et al., 2003). Nevertheless, Mani et al. (Mani et al., 2010) argued that the compressible-liquid mechanism cannot explain dry surface splashing when the impact velocity is low. Bussmann et al. (Bussmann et al., 2000) simulated droplet impact on dry surface, they artificially perturbed the velocity of the fluid near the solid surface to produce secondary droplets. Mandre and Brenner (Mandre \& Brenner, 2012) argued that splashing occurs through a two-stage process: in the first stage, the liquid droplet ejects a thin sheet before it contacts the surface; in the second stage, the sheet contacts the surface and viscous forces can deflect the sheet upwards to cause a splash. Lian et al. (Lian et al., 2014) numerically confirmed that a thin sheet lifts off to cause splashing but they did not observe the thin air layer between the droplet an the solid surface.

Surrounding air initially was not considered to affect the droplet impact (Bussmann et al., 2000). However, Xu et al. (Xu et al., 2005) and Latka et al. (Latka et al., 2012) found that air plays a crucial role in determining the splashing behavior: a low pressure (low air density equivalently) can effectively suppress the splashing. Riboux and Gordillo (Riboux \& Gordillo, 2014; Riboux \& Gordillo, 2015) proposed a model to predict drop splashing with the consideration of ambient air. Numerical simulation by Lian et al. (Lian et al., 2014) further confirmed that the splashing can be suppressed when the air density is lowered. Schroll et al. (Schroll et al., 2010) simulated droplet impact at low air density, but their work did not show droplet splashing at higher air density, hence it was not sufficient to conclude that droplet spreading was caused by lowering the air density. 
Droplet impact on thin liquid film has been studied experimentally (Yarin \& Weiss, 1995; Cossali et al., 1997; Wang \& Chen, 2000; Rioboo et al., 2003) and the threshold for thin liquid film splashing are revealed. For example, Yarin and Weiss (Yarin \& Weiss, 1995) proposed the threshold velocity for thin liquid film droplet splashing:

$$
V_{0 S}=18\left(\frac{\sigma}{\rho}\right)^{1 / 4} v^{1 / 8} f^{3 / 8}
$$

where $f$ is the frequency of incoming droplets. For single droplet splashing, $f$ can be replaced with $V_{0} / D_{0}$. Their study showed that the droplet diameter has no effect on the splashing threshold and the liquid film thickness is less important. Cossali et al. (Cossali et al., 1997) proposed the threshold $K$ value for droplet splashing on thin liquid film:

$$
K_{S}=2100+5880\left(\frac{h}{D_{0}}\right)^{1.44} \quad \text { for } 0.1<\frac{h}{D_{0}}<1 \text { and } O h>0.007
$$

where the thickness of the liquid film, $h$, is considered. However, the equation is only valid for $h / D_{0}>0.1$. For $h / D_{0}<0.1$, experimental data (Wang \& Chen, 2000; Rioboo et al., 2003) established the threshold $K_{S} \approx 400$.

In this paper, we investigate droplet impact on both dry and wet surfaces and splashing phenomena using the moment of fluid (MOF) method. The MOF method (Dyadechko \& Shashkov, 2005; Ahn \& Shashkov, 2007) is an extension of the volume of fluid (VOF) method (Schofield et al., 2008) in which the centroid of each material in a computational cell is simultaneously integrated along with the volume fraction for each material. The MOF reconstruction uses both the volume fraction and centroid for each material in order to determine the interface(s) in a cell. It has demonstrated that MOF reconstruction of a single interface in a cell is more accurate than the volume of fluid reconstruction (Jemison 
et al., 2012). The MOF method has also been shown to be more accurate than VOF methods for reconstructing multiple interfaces in a given cell. As with the single interface reconstruction the added centroid information in a cell results in a more accurate reconstruction of multiple interfaces. Because MOF has more degrees of freedom than the VOF, the computational cost is higher than VOF. In this paper, we focus on high speed impact on both dry and wet surfaces which have not been thoroughly investigated. The effect of ambient air on splashing is carefully studied to highlight the different splashing mechanisms in dry and wet surface impact. In this work, a dynamic contact model is implemented to handle the moving contact line. The following of this paper is structured as follows: first we present the numerical method, then we validate our code by comparing with available experimental data, next we present the low-speed impact study, and last we discuss the results of high speed impact on dry surface and thin liquid film to highlight the different roles ambient air played in splashing.

\subsection{Results and Discussions}

The numerical methods are first validated. Then simulation results of the ambient air effect on low and high-speed drop impacts on dry surfaces are presented. Last, the ambient air effect on drop impact on thin liquid film is studied. Table 1 summarizes the parameters for all the simulation cases in this section. 
Table 1. Summary of the parameters in numerical simulations

\begin{tabular}{|c|c|c|c|c|c|c|c|c|}
\hline & $\begin{array}{l}\text { Drop } \\
\text { size }\end{array}$ & $\begin{array}{l}\text { Liquid } \\
\text { type }\end{array}$ & $\begin{array}{l}\text { Impact } \\
\text { velocity }\end{array}$ & $\begin{array}{l}\text { Surface } \\
\text { property }\end{array}$ & $W e$ & $R e$ & $K$ & $\begin{array}{l}\text { Ambient air } \\
\text { density }\end{array}$ \\
\hline $\begin{array}{l}\text { A. Code } \\
\text { validation 1: } \\
\text { drop deposition } \\
\text { on a hydrophilic } \\
\text { surface }\end{array}$ & $\begin{array}{l}3.6 \\
\mathrm{~mm}\end{array}$ & water & $0.77 \mathrm{~m} / \mathrm{s}$ & $\begin{array}{l}\text { dry } \\
(\text { equilibrium } \\
\text { contact angle = } \\
\left.87.4^{\circ}\right)\end{array}$ & 30 & 2761 & 39 & $1.225 \mathrm{~kg} / \mathrm{m}^{3}$ \\
\hline $\begin{array}{l}\text { B. Code } \\
\text { validation 2: } \\
\text { drop complete } \\
\text { rebound on a } \\
\text { hydrophobic } \\
\text { surface }\end{array}$ & $\begin{array}{l}2.5 \\
\mathrm{~mm}\end{array}$ & water & $1.6 \mathrm{~m} / \mathrm{s}$ & $\begin{array}{l}\text { dry } \\
\text { (equilibrium } \\
\text { contact angle } \approx \\
\left.160^{\circ}\right)\end{array}$ & 89 & 3984 & 75 & $1.225 \mathrm{~kg} / \mathrm{m}^{3}$ \\
\hline $\begin{array}{l}\text { C. Code } \\
\text { validation 3: } \\
\text { drop impact on a } \\
\text { thin liquid film }\end{array}$ & $\begin{array}{l}271 \\
\mu \mathrm{m}\end{array}$ & ethanol & $12.5 \mathrm{~m} / \mathrm{s}$ & $\begin{array}{l}30 \mu \mathrm{m} \text { ethanol } \\
\text { film }\end{array}$ & 1500 & 2227 & 266 & $1.225 \mathrm{~kg} / \mathrm{m}^{3}$ \\
\hline $\begin{array}{l}\text { D. Ambient air } \\
\text { effect on low- } \\
\text { speed drop } \\
\text { impact on dry } \\
\text { surfaces }\end{array}$ & $\begin{array}{l}3.4 \\
\mathrm{~mm}\end{array}$ & ethanol & $3.74 \mathrm{~m} / \mathrm{s}$ & $\begin{array}{l}\text { dry } \\
\text { (equilibrium } \\
\text { contact angle } \approx \\
10^{\circ} \text { ) }\end{array}$ & 1685 & 8361 & 393 & $1.225 \mathrm{~kg} / \mathrm{m}^{3}$ \\
\hline \multirow{2}{*}{$\begin{array}{l}\text { E. The effect of } \\
\text { ambient air } \\
\text { density on high } \\
\text { speed drop } \\
\text { impact }\end{array}$} & $20 \mu \mathrm{m}$ & & & & 695 & 996 & 148 & \multirow{2}{*}{$\begin{array}{l}1.225 \mathrm{~kg} / \mathrm{m}^{3} \\
\text { and } 0.49 \\
\mathrm{~kg} / \mathrm{m}^{3}\end{array}$} \\
\hline & $\begin{array}{l}200 \\
\mu \mathrm{m}\end{array}$ & water & $50 \mathrm{~m} / \mathrm{s}$ & $\begin{array}{l}\text { contact angle } \approx \\
\left.70^{\circ}\right)\end{array}$ & 6946 & 9960 & 830 & \\
\hline $\begin{array}{l}\text { F. Ambient air } \\
\text { effect on drop } \\
\text { impact on thin } \\
\text { liquid film }\end{array}$ & $50-\mu \mathrm{m}$ & water & $78 \mathrm{~m} / \mathrm{s}$ & $\begin{array}{l}2 \mu \mathrm{m} \text { water } \\
\text { film }\end{array}$ & 4226 & 3884 & 513 & $\begin{array}{l}1.225 \mathrm{~kg} / \mathrm{m}^{3} \\
\text { and } 0.49 \\
\mathrm{~kg} / \mathrm{m}^{3}\end{array}$ \\
\hline
\end{tabular}

\subsubsection{Code validation 1: drop deposition on a hydrophilic surface}

Our first case is based on the experiment conducted by Kim and Chun (Kim \& Chun, 2001).

In the experimental setup, a water drop of the diameter of $3.6 \mathrm{~mm}$ impacted on a smooth, dry solid surface at a terminal velocity of $0.77 \mathrm{~m} / \mathrm{s}$. The equilibrium contact angle was $87.4^{\circ}$ and the surface tensions between the gas, liquid and solid phases used in the simulation were $\sigma_{l g}=0.0728 \mathrm{~N} / \mathrm{m}, \sigma_{l s}=0.0695 \mathrm{~N} / \mathrm{m}$, and $\sigma_{s g}=0.0728 \mathrm{~N} / \mathrm{m}$. This led to 
deposition and the drop eventually reached its final static state. All the dynamic contact angle models in and the static contact model were used in our simulations for comparison purpose. Grid sensitivity analysis was conducted and a converged solution of maximum base diameter was obtained at about 60 cells per original drop diameter.

Figure 8 compares the drop shapes during the spreading and recoiling stages between numerical result using the model of Jiang et al. (Jiang et al., 1979) and experimental result. The numerical prediction closely matches the experimental result. The time histories of the base diameter of the wetted surface are plotted in Figure 9 using all contact angle models discussed in the previous section. The abscissa is the normalized time $t^{*}$ and the ordinate is the normalized wet surface diameter $D_{b}^{*}$, which are defined as

$$
\begin{gathered}
t^{*}=\frac{t}{\left(\rho D^{3} / \sigma\right)^{1 / 2}} \\
D_{b}^{*}=\frac{D_{b}}{D}
\end{gathered}
$$

where $t$ is the time, $\rho$ is the drop density, $D$ is the original drop diameter, $\sigma$ is the drop surface tension, and $D_{b}$ is the wet surface diameter. 

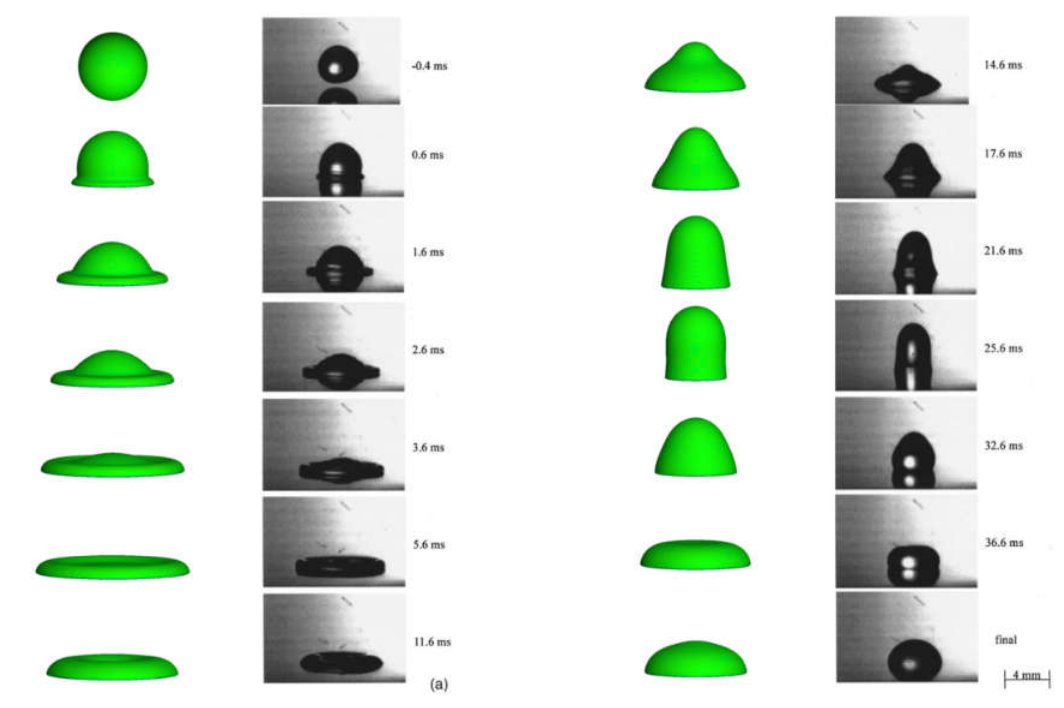

Figure 8. Comparison of drop shapes during the impact process. Numerical results are shown in green and experimental results (Kim \& Chun, 2001) in black.

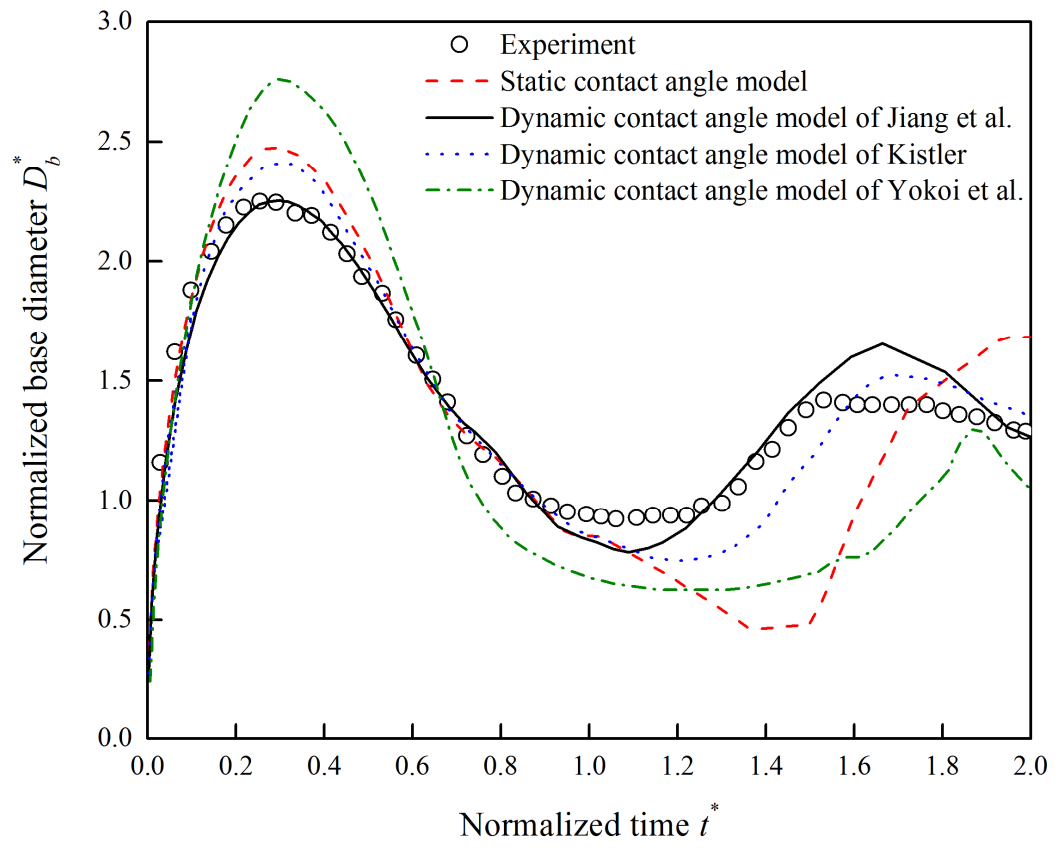

Figure 9. Comparison of wetted surface diameter between the experiment and simulation. 
During the initial spreading stage, the numerical results based on all the models match the experimental results well except that Yokoi's model over-predicts the maximum base diameter by approximately $20 \%$. During the following recoiling stage, good agreement is also observed for the static contact angle model, Jiang's model and Kistler's model. However, the static contact angle model and Kistler's model both predict smaller minimum base diameter which occurs at a later time instant. This test indicates that the dynamic contact angle model of Jiang et al. (Jiang et al., 1979) can produce better match with the experimental results in terms of the spreading diameter and shapes of the droplet after impact. Therefore, in the following simulations, the dynamic contact model will be used as default.

\subsubsection{Code validation 2: drop complete rebound on a hydrophobic surface}

This selected problem was originally studied by Azimi et al. (Azimi et al., 2013). In the experiment, a water drop impinged on a surface of nanograss-covered microposts sputtered with ceria and the drop experienced a complete rebound. The diameter of the water drop was $2.5 \mathrm{~mm}$ and the impact velocity was $1.6 \mathrm{~m} / \mathrm{s}$. The equilibrium contact angle was $160 \pm 2^{\circ}$ and the surface tensions between the gas, liquid and solid phases used in the simulation were $\sigma_{l g}=0.0728 \mathrm{~N} / \mathrm{m}, \sigma_{l s}=0.1412 \mathrm{~N} / \mathrm{m}$, and $\sigma_{s g}=0.0728 \mathrm{~N} / \mathrm{m}$. The resulting $W e$ is 89 and $R e$ is 3984 . The measured contact angle hysteresis was low $\left(<10^{\circ}\right)$ and it led to extremely high mobility. Complete rebounding was observed in the experiment.

Considering the problem was axisymmetric, the simulation was conducted in the $r-\theta$ coordinate system and revolved for 3D visualization. Grid sensitivity analysis was 
conducted and wetted surface diameter converged when 60 computational cells per the original drop diameter was used. Figure 10 compares the numerical simulations with the experimental results. The simulation showed good match with the experimental as it captured the complete rebound and the drop breakup thereafter.

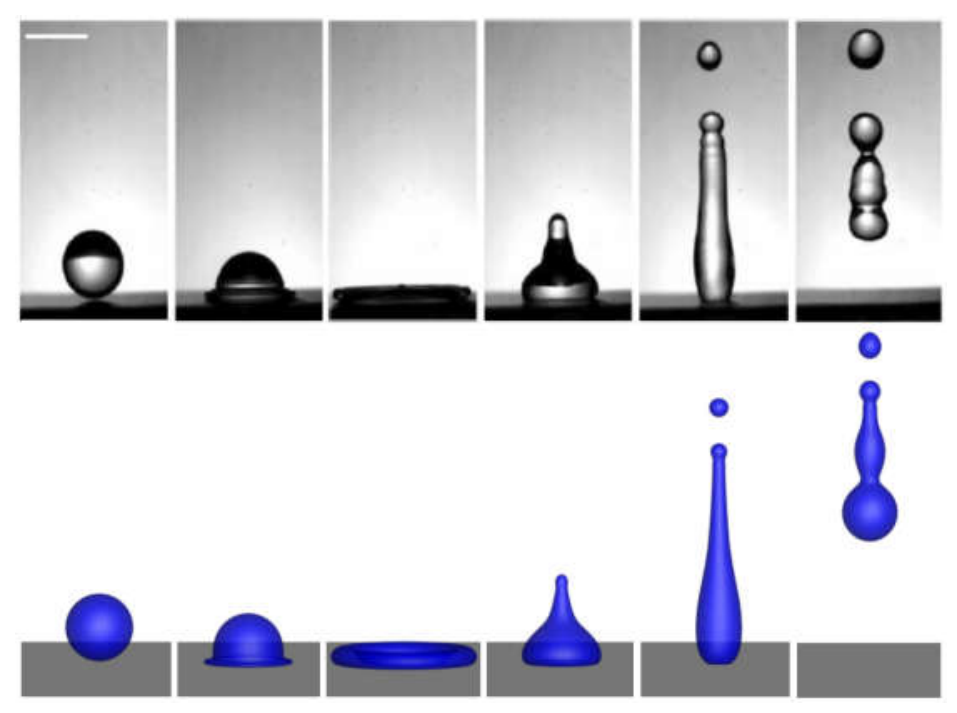

Figure 10. Comparison of the drop shapes in drop complete rebound phenomenon. Top: experiment by Azimi et al. (Azimi et al., 2013). Bottom: simulation.

\subsubsection{Code validation 3: drop impact on a thin liquid film}

We further validate the code with the simulation of drop impact on thin liquid film. The experiment (normal impact of successive ethanol drops on a solid surface) was conducted by Yarin and Weiss (Yarin \& Weiss, 1995). The drop diameter was $271 \mu \mathrm{m}$, the terminal impact velocity was $12.5 \mathrm{~m} / \mathrm{s}$, and the frequency of the impact was $19,973 \mathrm{~Hz}$, leading to We of 1500 and $R e$ of 2227. The liquid film thickness formed by successive drops was observed to be in the range of $20-50 \mu \mathrm{m}$, but the splashing threshold and crown formation 
were not sensitive to the film thickness (Cossali et al., 1997; Wang \& Chen, 2000; Rioboo et al., 2003). In our simulation, the ethanol drop size and impact velocity are the same as those in the experiment, which leads to the same $W e$ and Re. Film thicknesses of 20, 30, 40, and $50 \mu \mathrm{m}$ are simulated but no significant difference is noticed, which is in agreement with experimental observation. The simulations are conducted using both the $2 \mathrm{D}$ axisymmetric and 3D Cartesian grids. Note that a quarter computational domain in 3D simulation is used to utilize the symmetry. Grid sensitivity study shows that the equivalent resolution of about 430 cells per drop diameter yields converged solution of the diameter of the crown's rim. The 2D axisymmetric and 3D simulated results are shown in Figure 11 and Figure 12, respectively.

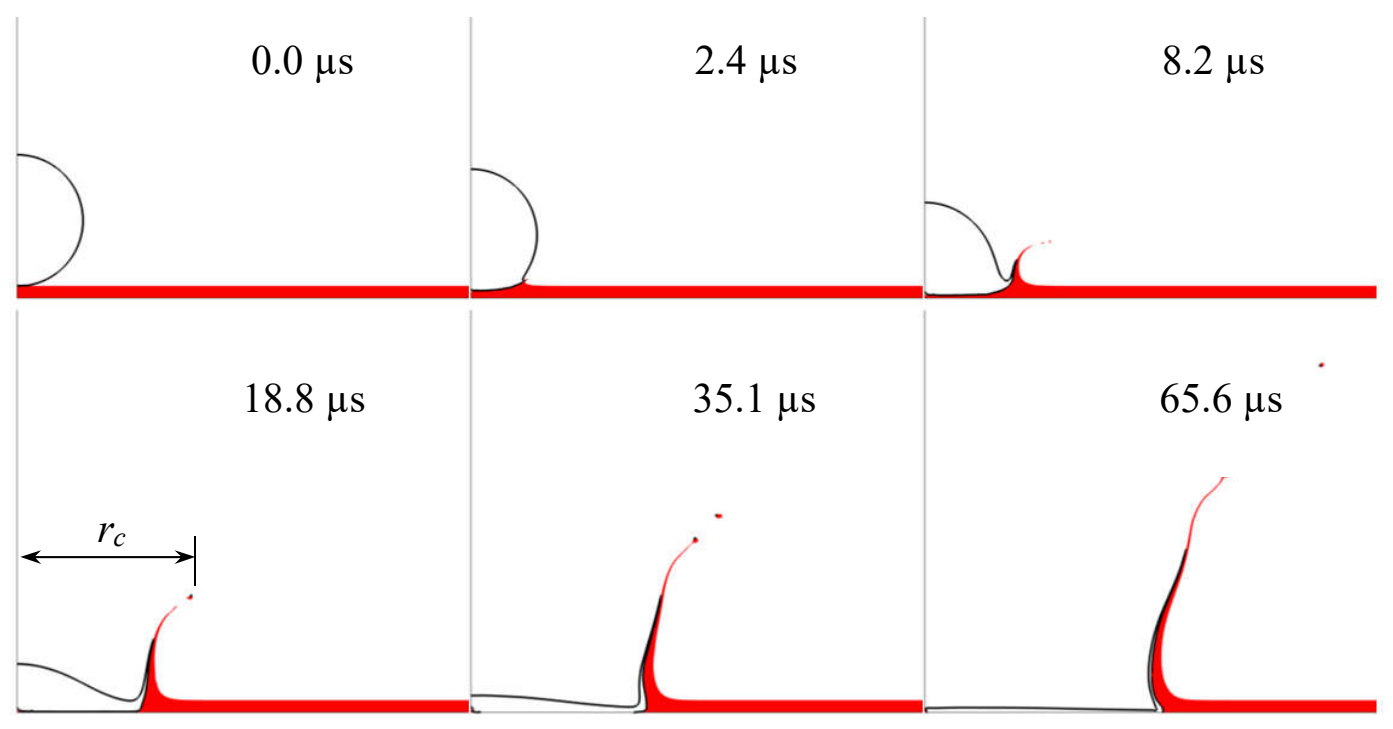

Figure 11. 2D axisymmetric simulation of ethanol drop impacts on a $30 \mu \mathrm{m}$ ethanol film with drop dimeter of $271 \mu \mathrm{m}$ and impact velocity of $12.5 \mathrm{~m} / \mathrm{s}(W e=1500, R e=2227)$. 


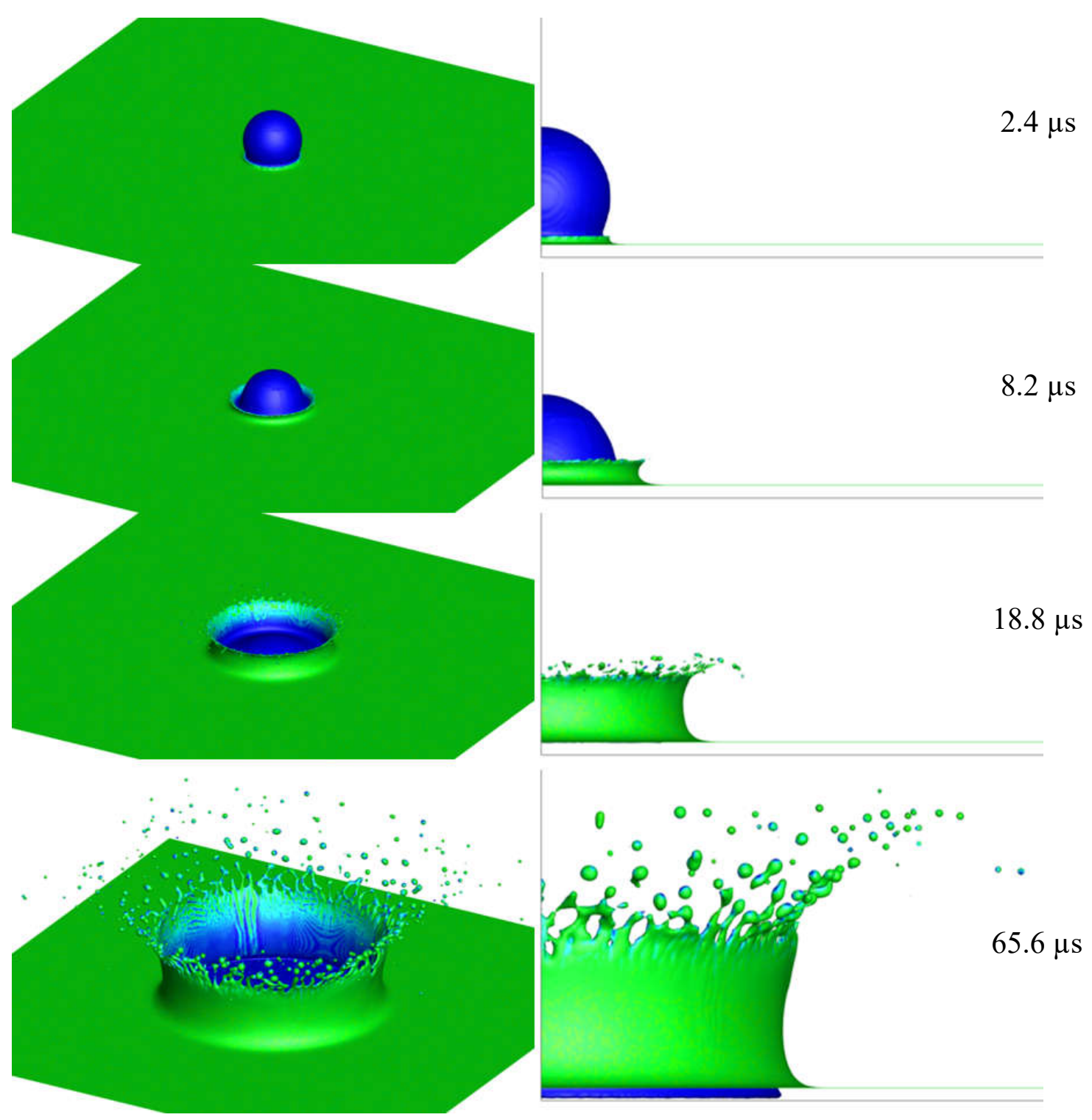

Figure 12. 3D simulation of ethanol drop impacts on a $30 \mu \mathrm{m}$ ethanol film with drop dimeter of $271 \mu \mathrm{m}$ and impact velocity of $12.5 \mathrm{~m} / \mathrm{s}(W e=1500, R e=2227)$.

It is clear from Figure 11 and Figure 12 as the drop impacts on the thin film, a crown is formed and it is pushed by the drop and moving outward. The crown movement can be 
predicted based on a theoretical quasi-1D model developed by Yarin and Weiss (Yarin \& Weiss, 1995) :

$$
\frac{r_{c}}{D}=\frac{V_{0}^{1 / 2}}{6^{1 / 4} \pi^{1 / 2} \nu^{1 / 8} D^{1 / 4} f^{3 / 8}}\left(\tau-\tau_{0}\right)^{1 / 2}
$$

where $r_{c}$ is the radius of crown's rim, $D$ is original drop diameter, $V_{0}$ is terminal impact velocity, $v$ is kinematic viscosity, $f$ is impact frequency, $\tau$ is dimensionless time $(\tau=2 \pi f t)$, and $\tau_{0}$ is the dimensionless shifting time $\left(\tau_{0}=2 \pi f t_{0}\right)$. The numerical, experimental, and theoretical results of the crown's rim are compared in Figure 13. The simulation result is plotted from the initiation of the crown. The crown's rim moves relatively fast at the beginning and then it reaches a relatively constant speed moving outward. The simulation shows good agreement with the experimental result. 


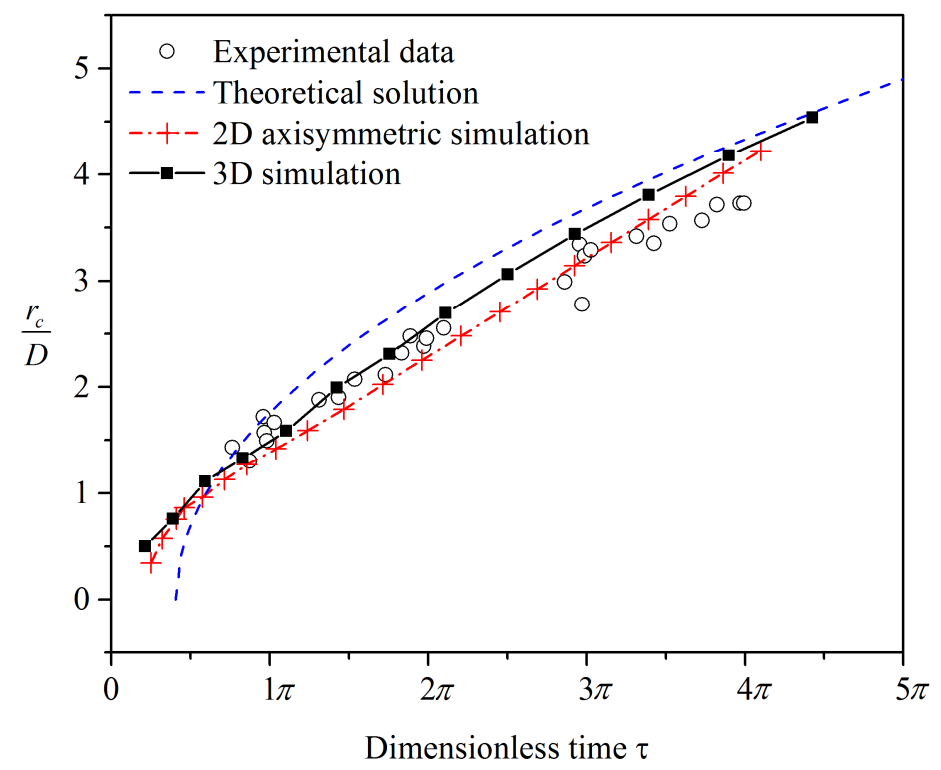

Figure 13. Comparison of the radius of crown's rim in ethanol drop impact on thin liquid film.

\subsubsection{Ambient air effect on low-speed drop impact on dry surfaces}

Xu et al. (Xu et al., 2005) and Latka et al. (Latka et al., 2012) found that ambient air pressure affected the splashing behavior: a low pressure can suppress splashing. In their experiments, splashing was observed when the ambient pressure was $100 \mathrm{kPa}$ but was suppressed at a low pressure of $13 \mathrm{kPa}$. In both cases, the $K$ value of 393 is much higher than the splashing threshold of Mundo et al. (Mundo et al., 1995).

Our simulation followed the experimental setup. The initial diameter of the ethanol drop is $3.4 \mathrm{~mm}$ and the terminal velocity is $3.74 \mathrm{~m} / \mathrm{s}$. The low air pressure is modeled with low air density by assuming air is ideal gas. In the experiments, the ambient temperature is kept the same for different air pressures, and the viscosity of the air and the surface tension of 
the liquid do not vary with the pressure changes. Therefore, it is the ambient air density not the pressure that affects the splashing behavior (low pressure can be the result of low temperature, but reducing the pressure without changing the density will have less effect on the splashing). The resulting dimensionless parameters are: $W e=1685, R e=8361$, and $K=393$. The equilibrium contact angle of the drop is $10^{\circ}$ and the surface tensions between the gas, liquid and solid phases are $\sigma_{l g}=0.0223 \mathrm{~N} / \mathrm{m}, \sigma_{l s}=0.00034 \mathrm{~N} / \mathrm{m}$, and $\sigma_{s g}=0.0223$ $\mathrm{N} / \mathrm{m}$. The dynamic contact angle model (Jiang et al., 1979) was used in the simulation.

Grid sensitivity analysis was conducted and a converged solution of the thickness of the ejected thin film was reached when there were approximately 3000 equivalent cells per diameter of the original drop. Figure 14 compares the numerical and experimental results at two time instants. A thin liquid film is formed soon after the drop hits the surface. The thin film is moving outward in the radial direction and then it is lifted from the solid surface, resulting in drop splashing. The thin film later breaks up into smaller secondary droplets. A close-up look of the drop splashing is shown in Figure 15. It can be seen that air is trapped between the drop and the solid surface, and later the entrained air contracts into a bubble, which is in agreement with experiment (van der Veen et al., 2012). However, air is not pushed toward the drop perimeter as the drop deforms further, indicating that splashing is not caused by the trapped air. 

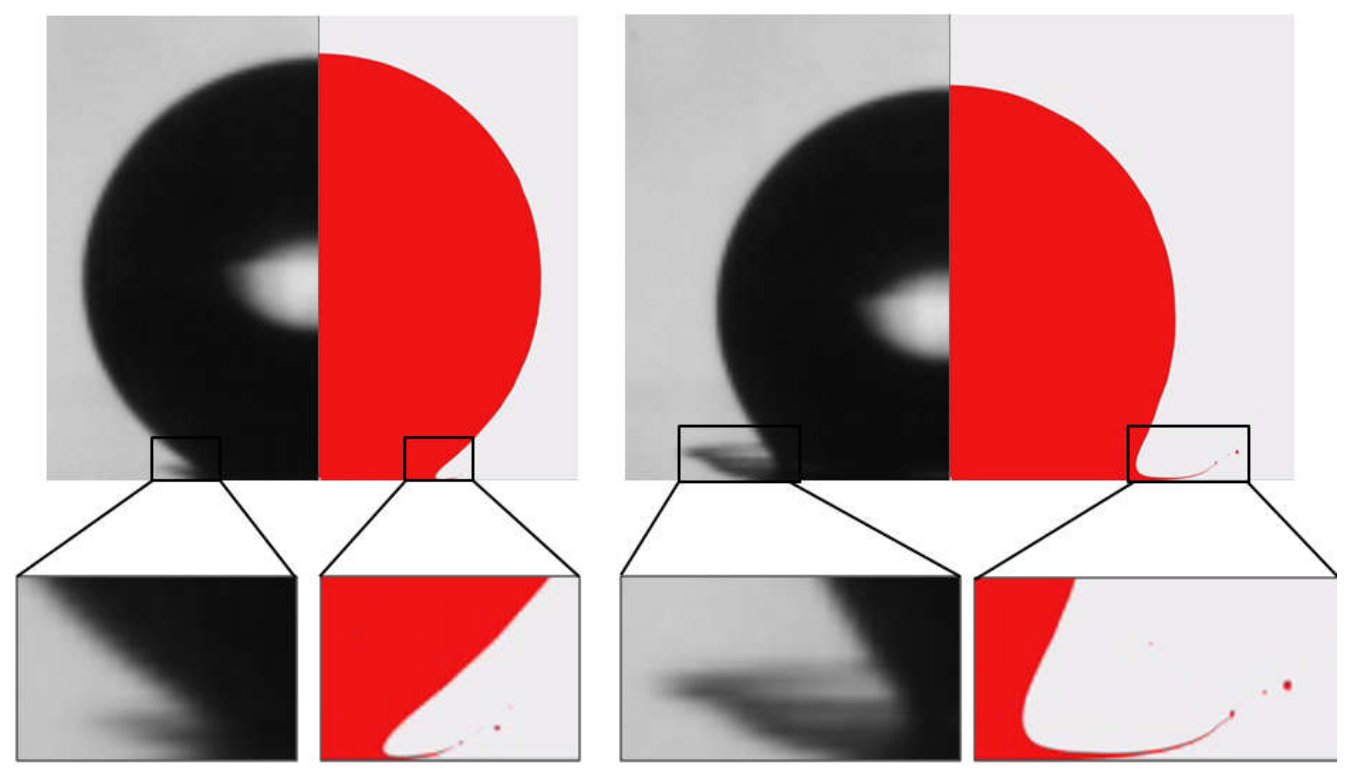

Figure 14. Droplet splashing on a solid surface. Red: numerical simulation; Black: experimental results (Xu et al., 2005). $W e=1685, R e=8361$, and $K=393$.

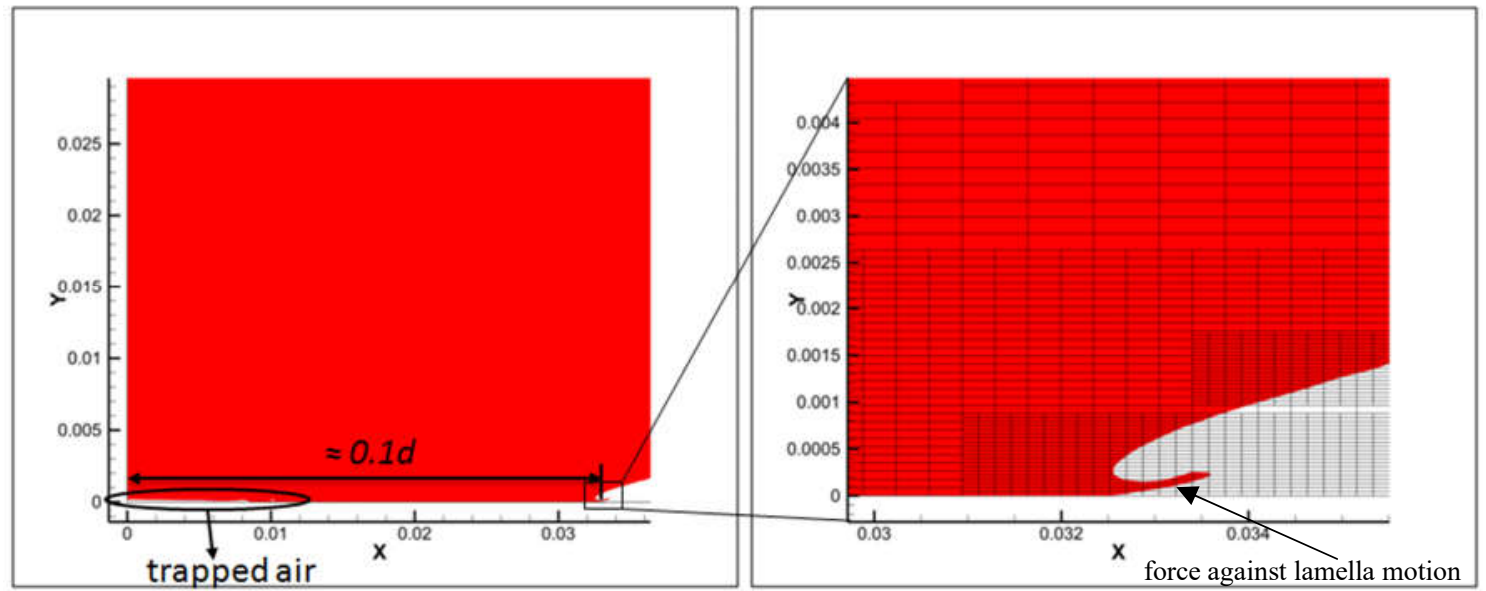

Figure 15. Close-up look of the trapped air and the lamella. The trapped air is not responsible for the splashing.

Simulation shows the splashed lamella has a thickness of approximately $1.5 \mu \mathrm{m}$ and a maximum speed of up to $30 \mathrm{~m} / \mathrm{s}$. Simulation also shows that the lamella close to the solid 
surface moves at a lower speed than that away from the wall, possibly due to the high shear stress on the layer next to the solid. As show in Figure 15, air is trapped under the lamella. When the lamella moves outward, the surrounding air exerts a resistance force against its motion and forces it to move upward and cause splashing. Since the aerodynamic forces on the lamella will be proportional to $\rho_{a i r} u^{2}$ ( $u$ is the lamella spreading speed), the air exerts force against the thin film motion and pushes the thin film upward to cause splashing.

To further validate our hypothesis, drop impact on solid surface with low ambient air pressure is simulated. The computational setting is the same as the previous one except the air density is $0.245 \mathrm{~kg} / \mathrm{m}^{3}$, which represents the ambient pressure of 0.2 atm (for air at 1 $\left.\mathrm{atm}, \rho_{\text {air }}=1.225 \mathrm{~kg} / \mathrm{m}^{3}\right)$. Since experiment has shown that viscosity and surface tension of air are insensitive to pressure change (Xu et al., 2005), the viscosity and surface tension are the same as those used in the previous test. Figure 16 compares the numerical results between the two ambient air densities. It is clear that drop splashing is completely suppressed at the low ambient air density, which is consistent with the experiment of $\mathrm{Xu}$ et al. (Xu et al., 2005). At the lower ambient pressure, the air density is low, hence, the force acting on the thin film, which is proportional to $\rho_{\text {air }} u^{2}$ and is smaller. Figure 17 shows the vorticity contours near the lamella for both cases. The vorticity magnitude is greater at $\rho_{\text {air }}=1.225 \mathrm{~kg} / \mathrm{m}^{3}$ than at $\rho_{\text {air }}=0.245 \mathrm{~kg} / \mathrm{m}^{3}$. Vortices are also observed near the splashed secondary droplets. 

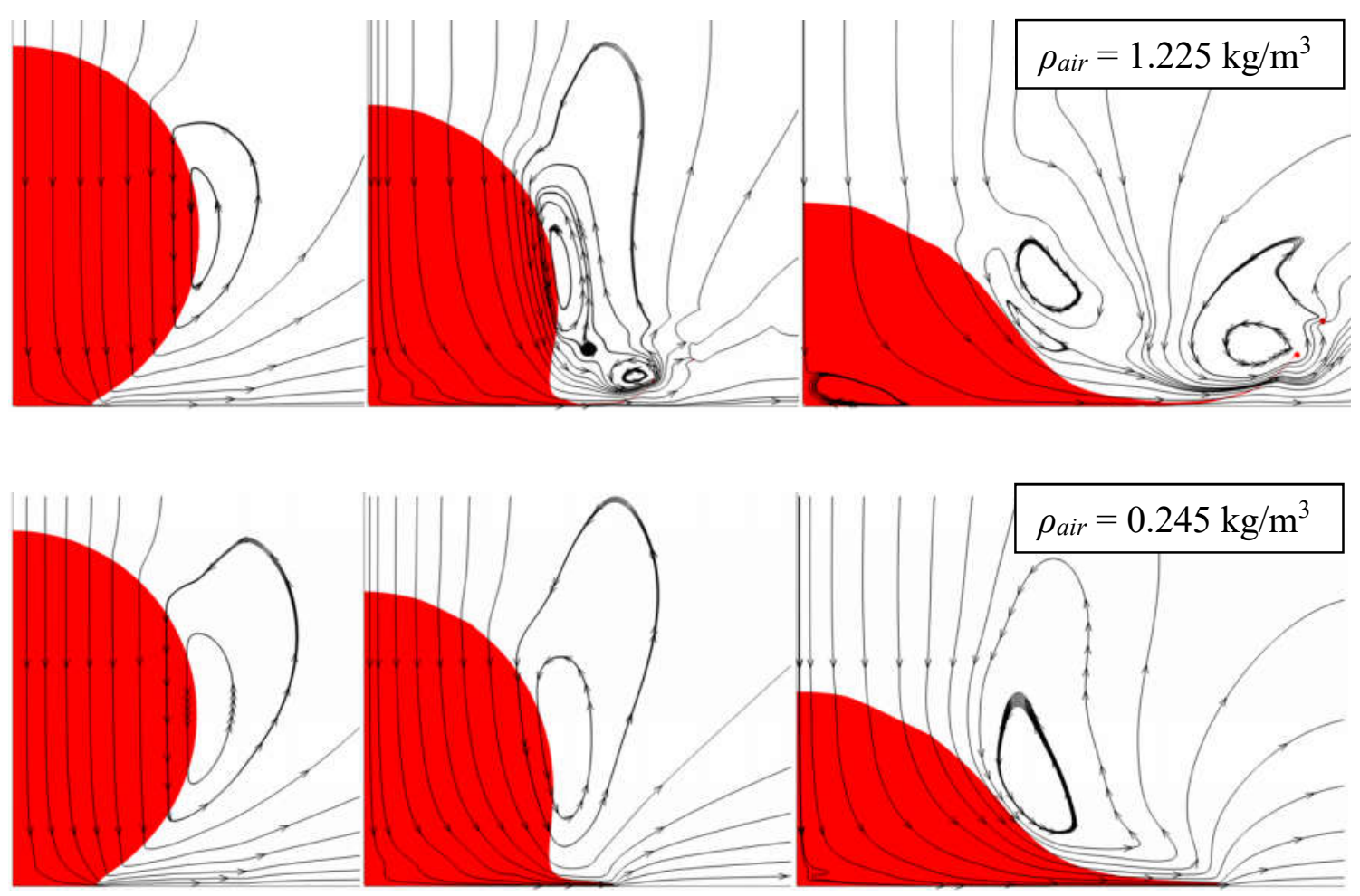

Figure 16. Comparison of droplet impact at $\rho_{\text {air }}=1.225 \mathrm{~kg} / \mathrm{m}^{3}$ and $0.245 \mathrm{~kg} / \mathrm{m}^{3}$. Splash occurs at high ambient air density but not at low density. 


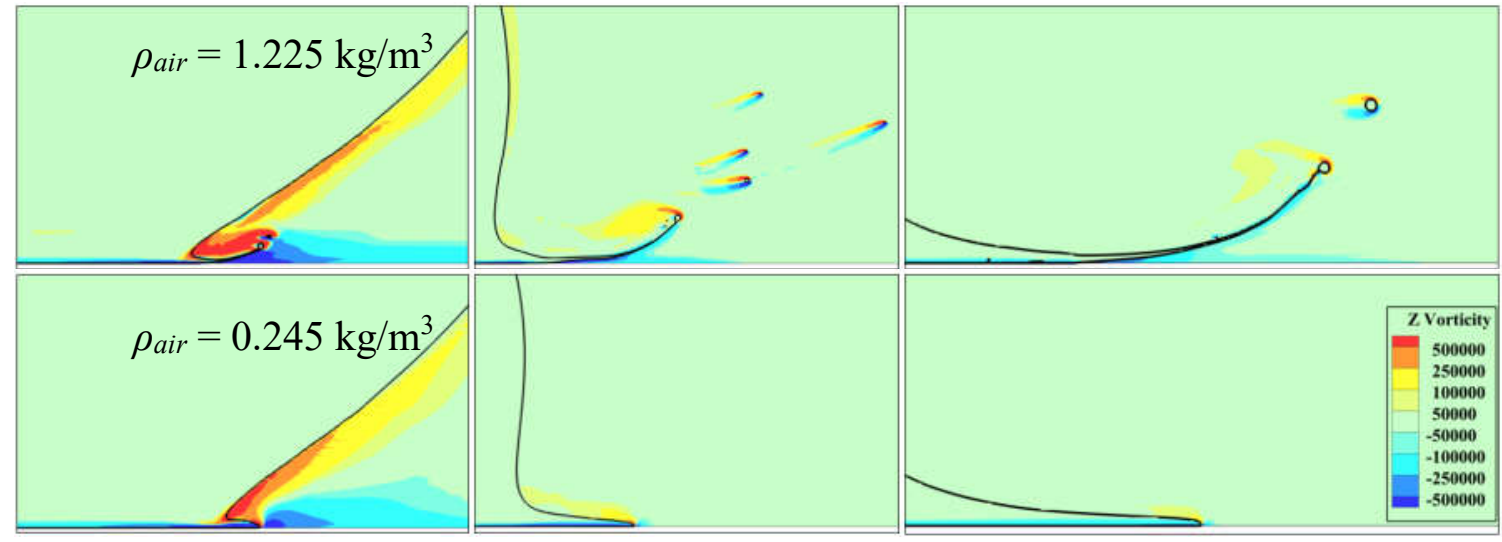

Figure 17. Vorticity contours near the lamella at $\rho_{\text {air }}=1.225 \mathrm{~kg} / \mathrm{m}^{3}$ and $0.245 \mathrm{~kg} / \mathrm{m}^{3}$

A recirculation zone is observed inside the drop after it impacts on the solid surface for both cases. The development of the recirculation at $\rho_{\text {air }}=1.225 \mathrm{~kg} / \mathrm{m}^{3}$ is shown in Figure 18. At the very early stage of droplet contact with the solid surface, the streamlines inside the drop are nearly parallel to each other and only bended at the proximity to the solid surface. Near the stagnation point a recirculation zone is formed and it grows in size. At $t$ $=0.336 \mathrm{~ms}$, secondary recirculation zones are formed near the solid surface. Near the stagnation point, air is trapped and squeezed into bubbles, as shown in Figure 19. The liquid flows around the air bubble and flow near the wall has extremely low velocity due to viscous effect. For drop impact at $\rho_{\text {air }}=0.245 \mathrm{~kg} / \mathrm{m}^{3}$, as shown in Figure 20, recirculation is observed but it is formed at a much later stage (at $\mathrm{t}=0.551 \mathrm{~ms}$ for $\rho_{\text {air }}=0.245 \mathrm{~kg} / \mathrm{m}^{3}$, while at $\mathrm{t}=0.236 \mathrm{~ms}$ for $\rho_{\text {air }}=1.225 \mathrm{~kg} / \mathrm{m}^{3}$ ). The recirculation zone size is much smaller than that at $\rho_{\text {air }}=1.225 \mathrm{~kg} / \mathrm{m}^{3}$. 


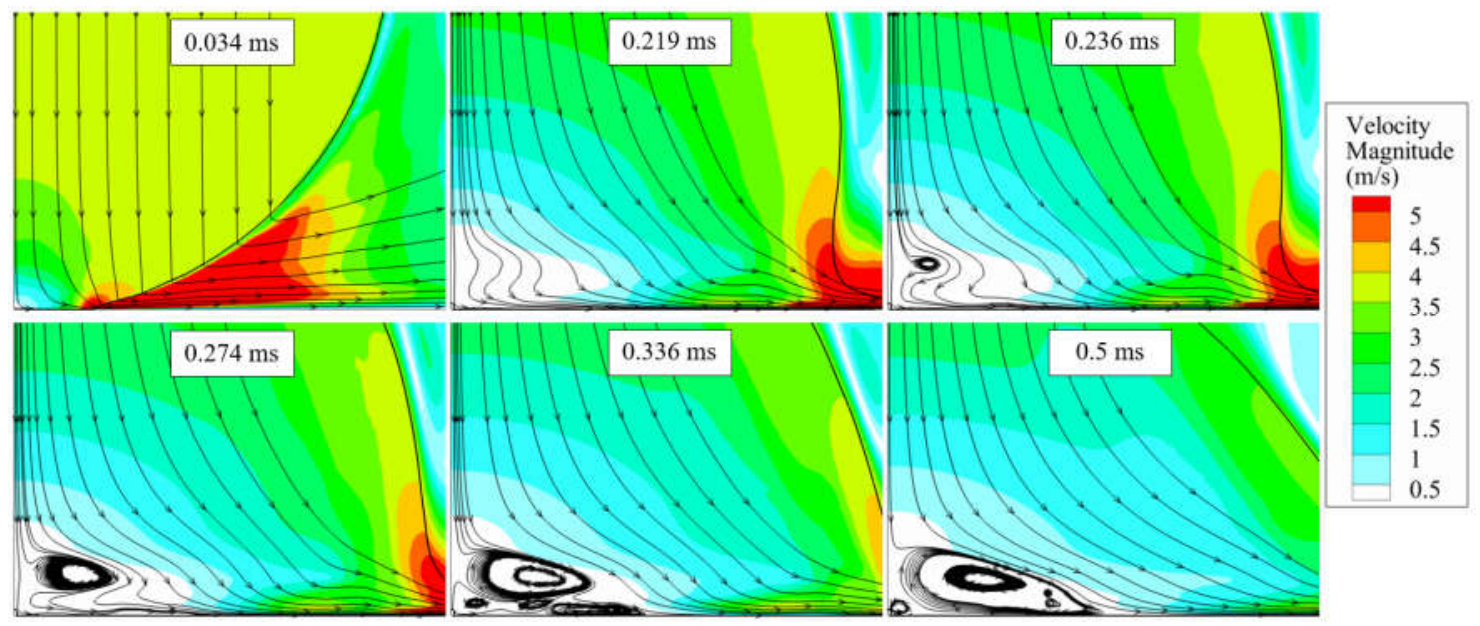

Figure 18. Development of a recirculation region inside the drop at $\rho_{\text {air }}=1.225 \mathrm{~kg} / \mathrm{m}^{3}$.

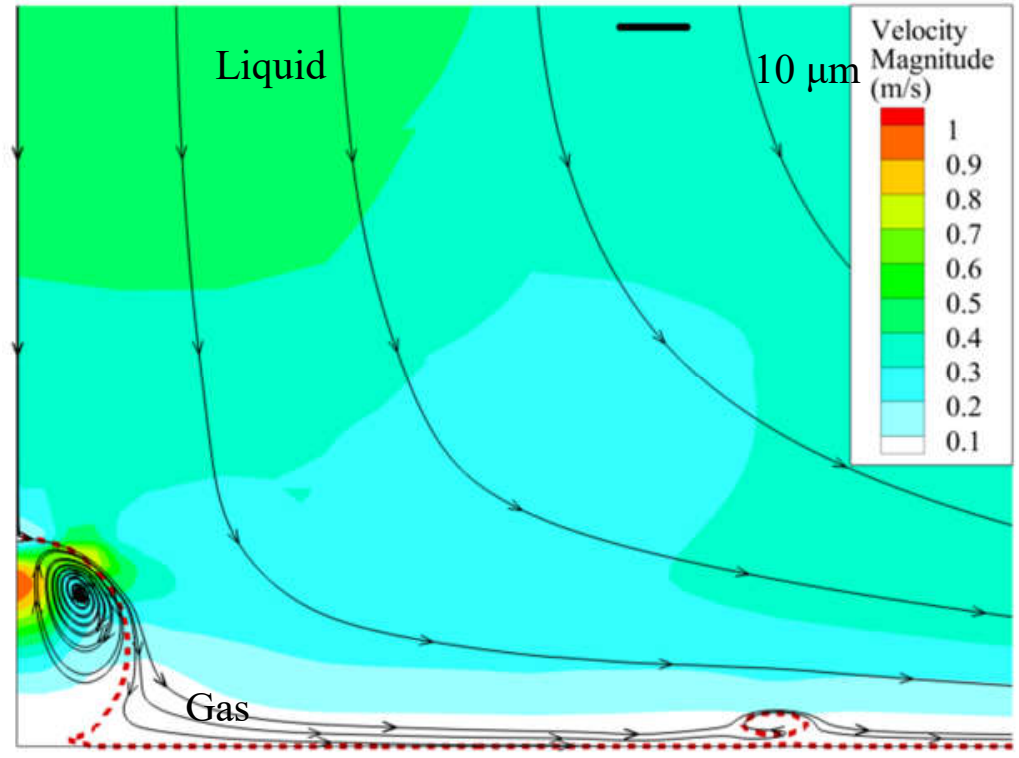

Figure 19. Air bubble is trapped at the center of the drop at $\mathrm{t}=0.12 \mathrm{~ms}$ for drop impact at $\rho_{\text {air }}=1.225 \mathrm{~kg} / \mathrm{m}^{3}$. The interface between liquid and gas is shown by dashed red lines. 


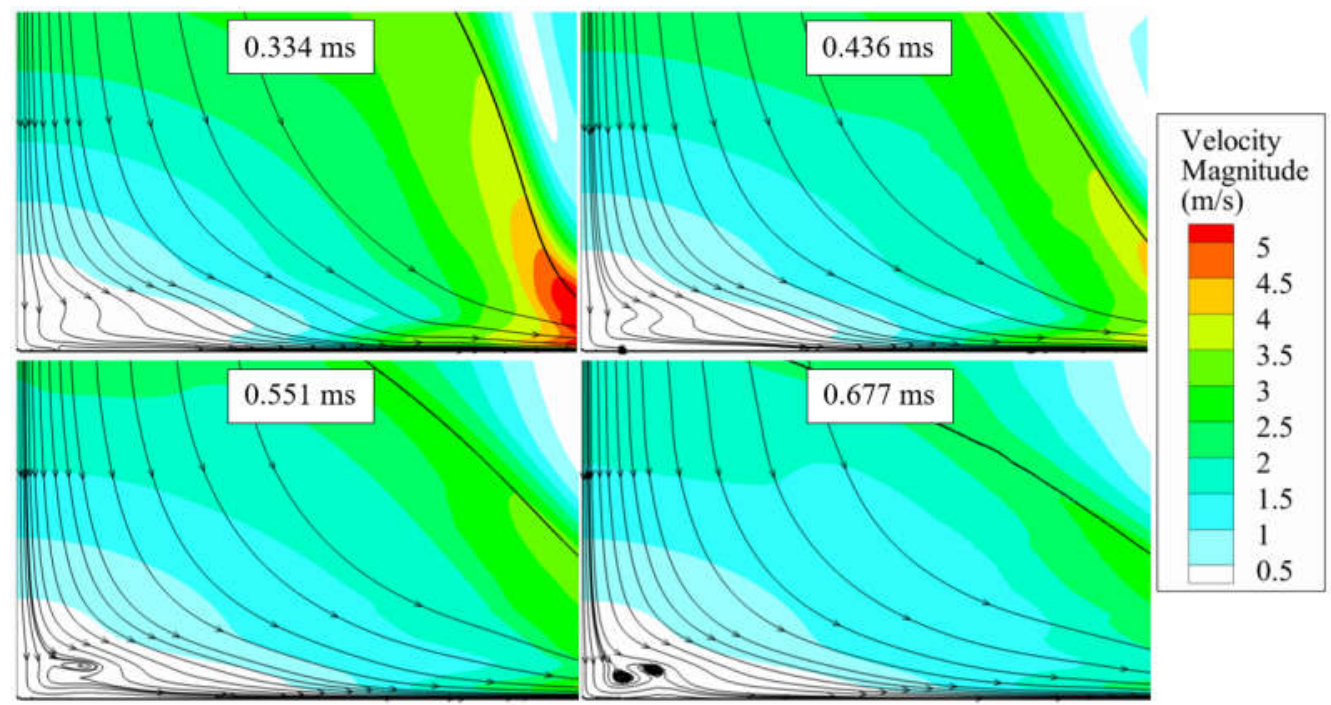

Figure 20. Development of recirculation region for drop impact at $\rho_{\text {air }}=0.245 \mathrm{~kg} / \mathrm{m}^{3}$.

\subsubsection{The effect of ambient air density on high speed drop impact}

We further study the effect of ambient air density on high speed drop impact. The water droplet has a diameter of $200 \mu \mathrm{m}$, which belongs to the supercooled large droplet category. The impact speed is $50 \mathrm{~m} / \mathrm{s}$. The solid surface is assumed to be aluminum, which is commonly used for aircraft construction. The equilibrium contact angle is $70^{\circ}$ and the surface tensions between the gas, liquid and solid phases used in the simulation were $\sigma_{l g}=$ $0.0728 \mathrm{~N} / \mathrm{m}, \sigma_{l s}=0.0479 \mathrm{~N} / \mathrm{m}$, and $\sigma_{s g}=0.0728 \mathrm{~N} / \mathrm{m}$. For comparison purpose, we also simulate the impact of a $20-\mu \mathrm{m}$ diameter cloud-sized droplet, which is encountered in most icing conditions. We assume an axisymmetric condition of the impact and the $\mathrm{r}-\theta$ coordinate system is used. The ambient air densities of $1.225 \mathrm{~kg} / \mathrm{m}^{3}$ and $0.49 \mathrm{~kg} / \mathrm{m}^{3}$ are considered to represent the air pressures of $1 \mathrm{~atm}$ and $0.4 \mathrm{~atm}$. The latter pressure corresponds to a flight altitude of 7000 meters. For the small droplet of diameter of $20 \mu \mathrm{m}$, 
$W e=695, R e=996$, and $K=148$; for the large droplet of diameter of $200 \mu \mathrm{m}, W e=6946$, $R e=9960$, and $K=830$. For both cases, the $K$ values exceed the splashing threshold of Mundo et al. (Mundo et al., 1995). In all simulations for both cases, two-level grid refinement is used, resulting in an equivalent resolution of approximately 2000 cells per diameter of the original droplet. The simulation results are summarized in Figure 21 and Figure 22. The normalized time is defined as $t^{*}=\frac{V_{0} t}{D_{0}}$ here. At $\rho_{\text {air }}=1.225 \mathrm{~kg} / \mathrm{m}^{3}$, splashing occurs for both droplets but the splashing is more prominent for the larger droplet. Note that in these two figures the two droplets are not shown using the same scale. When the ambient air density is lowered to $0.49 \mathrm{~kg} / \mathrm{m}^{3}$, splashing is attenuated for the larger droplet and is completely suppressed for the smaller droplet, suggesting that the surrounding air density affects the droplet impact behaviors. 


$$
t^{*}=0.075 \quad t^{*}=0.225 \quad t^{*}=0.375
$$
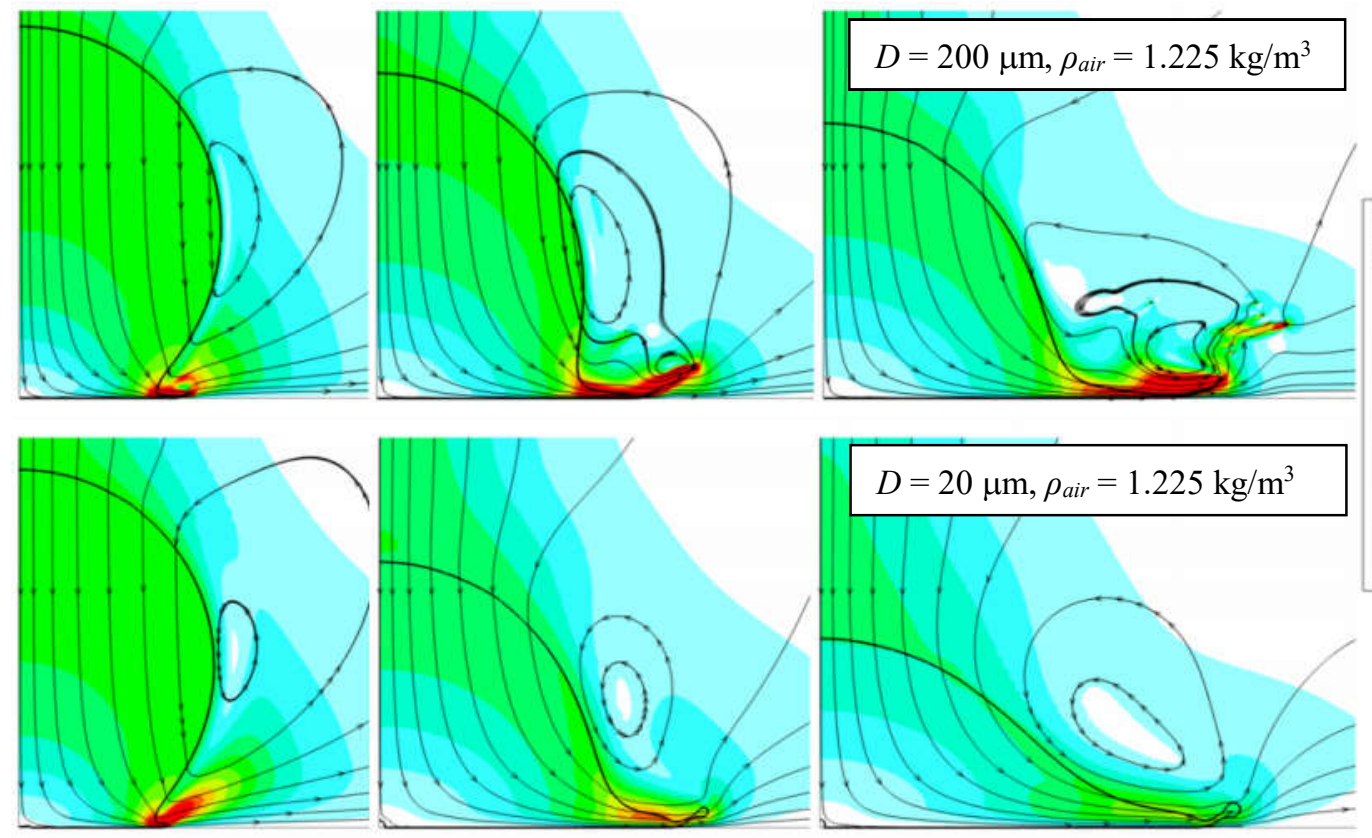

Velocity

Magnitude $(\mathrm{m} / \mathrm{s})$

Figure 21. Comparison of $200-\mu \mathrm{m}$ and $20-\mu \mathrm{m}$ droplet impact at $\rho_{\text {air }}=1.225 \mathrm{~kg} / \mathrm{m}^{3}$.
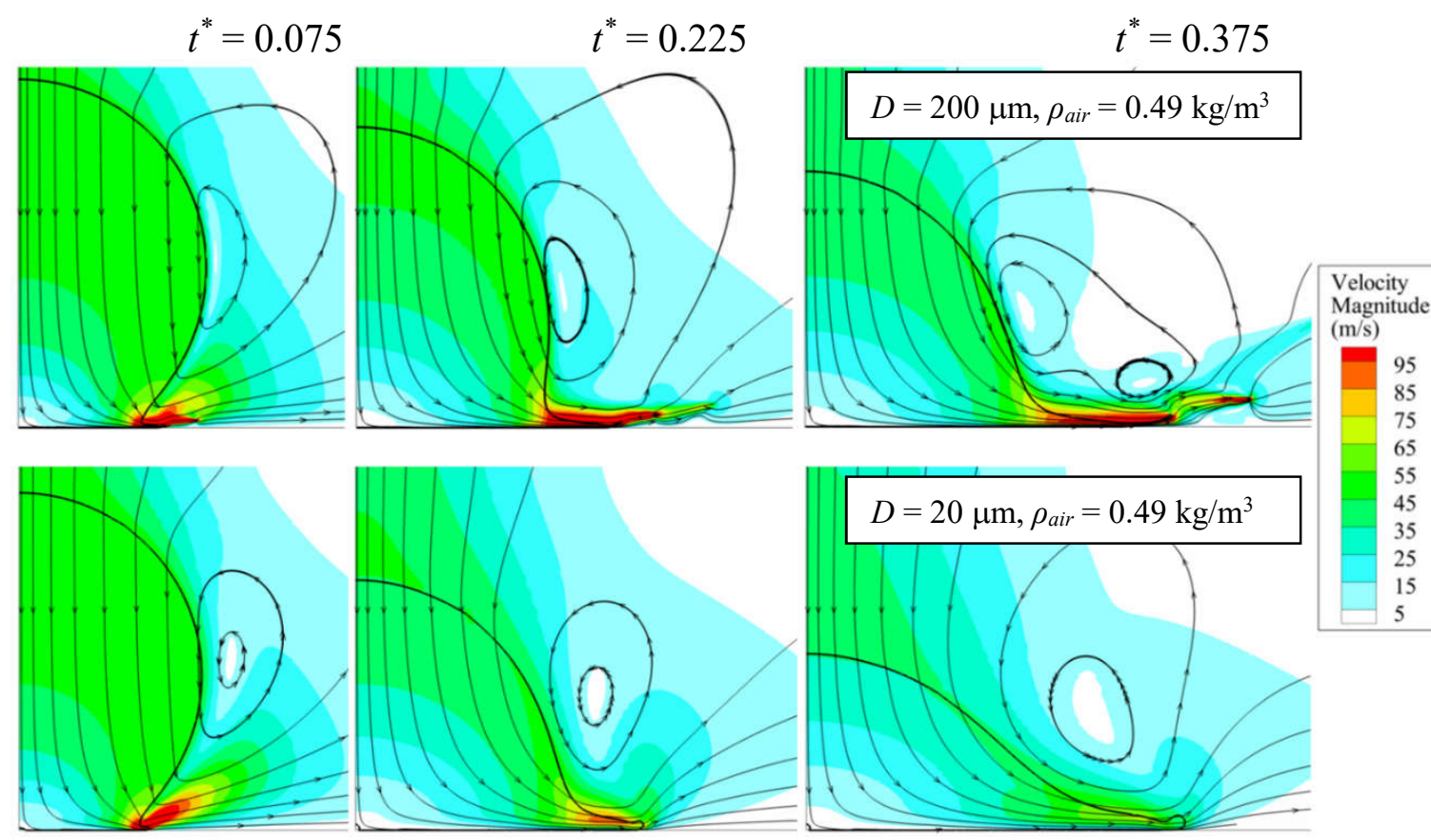

Figure 22. Comparison of $200-\mu \mathrm{m}$ and $20-\mu \mathrm{m}$ droplet impact at $\rho_{\text {air }}=0.49 \mathrm{~kg} / \mathrm{m}^{3}$. 
Figure 23 shows the simulation result of $200-\mu \mathrm{m}$ droplet impact at the ambient air density of $1.225 \mathrm{~kg} / \mathrm{m}^{3}$ in $3 \mathrm{D}$ coordinate system. The equivalent grid resolution is 320 cells per droplet diameter. The $3 \mathrm{D}$ simulation captures the expanding thin film lifts up at the front edge and breaks into secondary droplets.
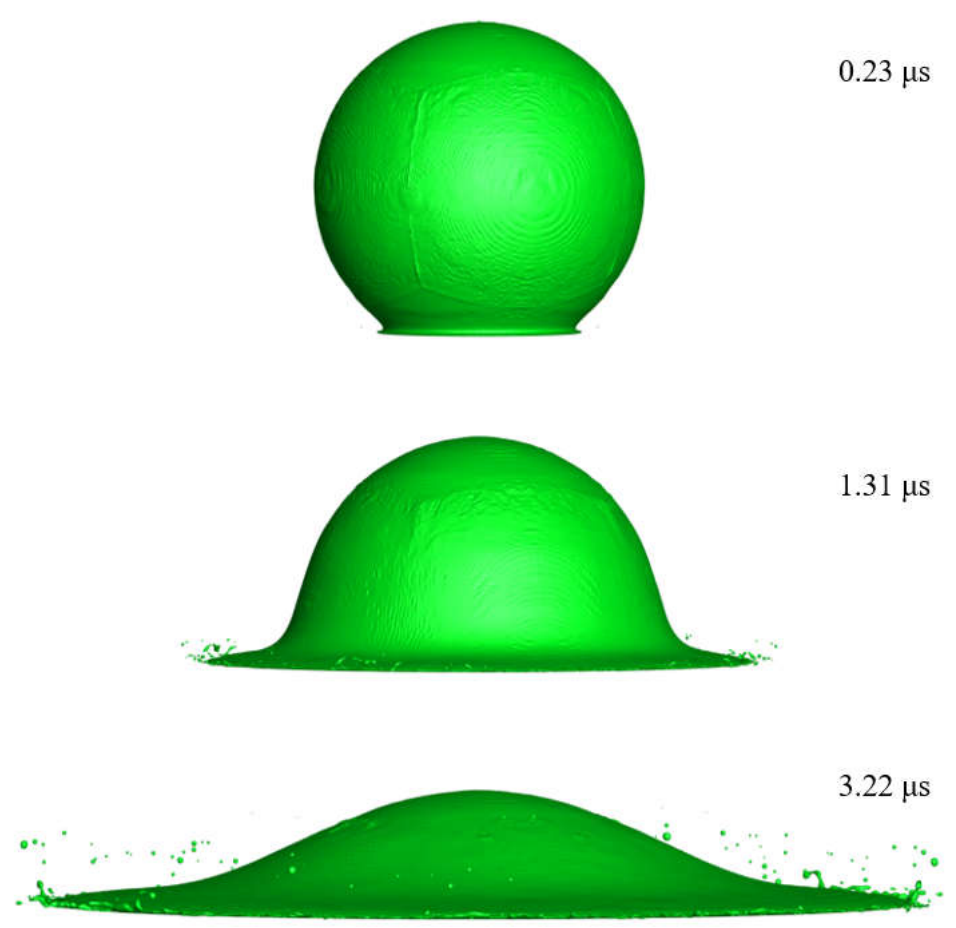

Figure 23. 3D simulation of $200-\mu \mathrm{m}$ droplet impact at $\rho_{\text {air }}=1.225 \mathrm{~kg} / \mathrm{m}^{3}$.

\subsubsection{Ambient air effect on drop impact on thin liquid film}

The impact of a $50-\mu \mathrm{m}$ diameter water droplet onto a uniform thickness water film with the impact velocity of $78 \mathrm{~m} / \mathrm{s}$ is simulated. Zhang and $\mathrm{Hu}$ (Zhang \& $\mathrm{Hu}, 2014$ ) experimentally studied the wind-driven water film on a NACA 0012 airfoil and measured the thickness of the water film. For the tested wind speed between 10 to $30 \mathrm{~m} / \mathrm{s}$, the 
measured film thickness varied from approximately 2 to $40 \mu \mathrm{m}$. The film thickness decreases with the wind speed. For the impact velocity of $78 \mathrm{~m} / \mathrm{s}$ (assume wind and droplet have the same speed), we assume a uniform film thickness of $2 \mu \mathrm{m}$.

An equivalent grid resolution of 684 cells per original droplet diameter is used here according to convergence study (4-level grid refinement with initial 42 cells per drop diameter). Figure 24 shows the $2 \mathrm{D}$ axisymmetric simulation results at the ambient air densities of $1.225 \mathrm{~kg} / \mathrm{m}^{3}$ and $0.49 \mathrm{~kg} / \mathrm{m}^{3}$. No significant difference is observed from lowering the ambient air density: unlike the dry surface cases splashing is not suppressed at lower air density. Nevertheless, the tip of the lamella at the lower air density moves slightly faster outward compared with the case at the higher air density. As indicated by the dashed vertical lines in Figure 24, the tip of the expanding lamella at lower air density is ahead of that at higher air density while the bases of the lamella are at the same position. The reason is that the lower density air exerts less resistance on the expanding lamella. Also, the lower density case shows more secondary droplets as the tip of the lamella. Figure 25 compares the radius of crown's rim, $\frac{r_{c}}{D}$, with the theoretical model developed by Yarin and Weiss (Yarin \& Weiss, 1995). For both $\rho_{\text {air }}=1.225 \mathrm{~kg} / \mathrm{m}^{3}$ and $\rho_{\text {air }}=0.49 \mathrm{~kg} / \mathrm{m}^{3}$ cases, the crowns occur earlier than the theoretical prediction and move at the same velocity at the beginning stage. After $\mathrm{t}=0.5 \mu \mathrm{s}$, the crown's rim at lower air density outruns that at higher air density. 


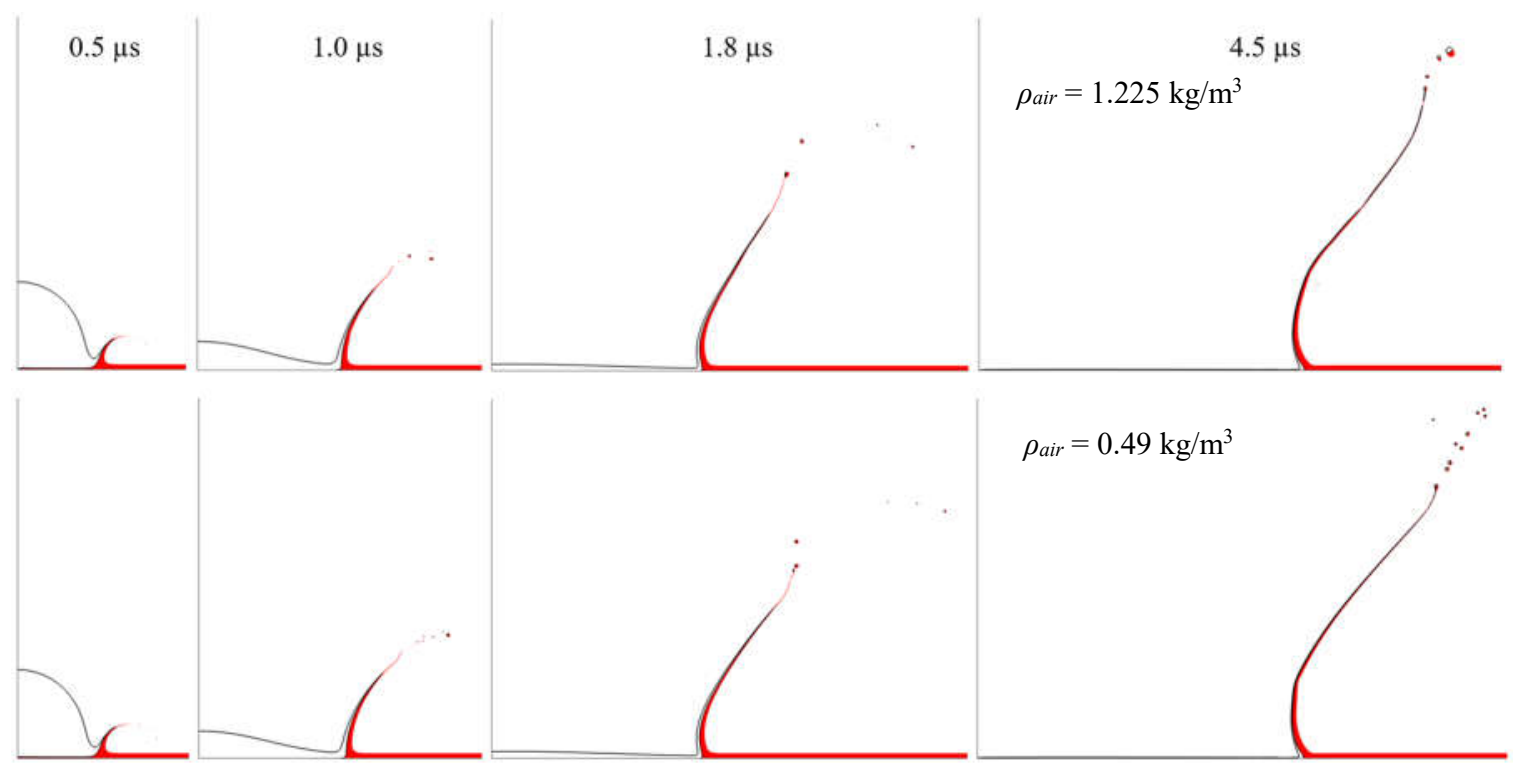

Figure 24. Comparison of droplet impact on thin liquid film at different ambient air densities.

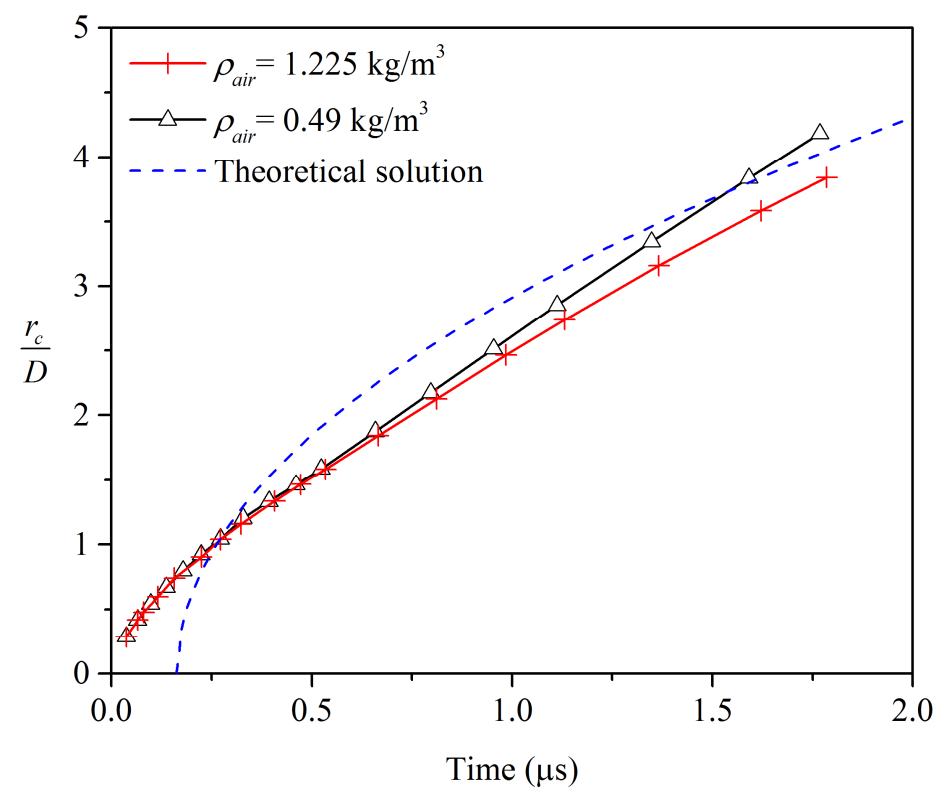

Figure 25. Comparison of the radius of crown's rim at different air densities with theoretical solution. 
The thin film splashing mechanism is significantly different from dry surface splashing: when the droplet impacts on the thin film it expels the film from the surface and pushes it move outward. A lamella is formed and moves upward. The lamella consists of liquid from both the thin film and the droplet (Figure 24). As the droplet further impacts the surface it keeps feeding the lamella and pushing the thin film outward until the lamella breaks up. After the lamella breaks up, partial volume of liquid from the droplet sticks to the surface and the rest moves away from the surface, and the mass ratio between the splashed liquid $\left(m_{s}\right)$ and the original droplet $\left(m_{o}\right)$ is defined as the mass loss ratio due to splashing, $\frac{m_{s}}{m_{o}}$. The calculated mass loss from splashing is $76.5 \%$ in this case, which is much higher than the upper limit (25\%) of dry surface splashing. Figure 26 compares the mass loss from splashing on wet surface as a function of $K$ value with existing empirical models. Although the models of Samenfink et al. (Samenfink et al., 1999) and Schmehl et al. (Schmehl et al., 1999) predict that splashing occurs at the same $K$ value, the model of Samenfink et al. (Samenfink et al., 1999) predicts higher splashed mass than the model of Schmehl et al. (Schmehl et al., 1999). Figure 26 also indicates that our result matches well with the prediction of Honsek et al. (Honsek et al., 2008) with the relative error of 4.8\%. 


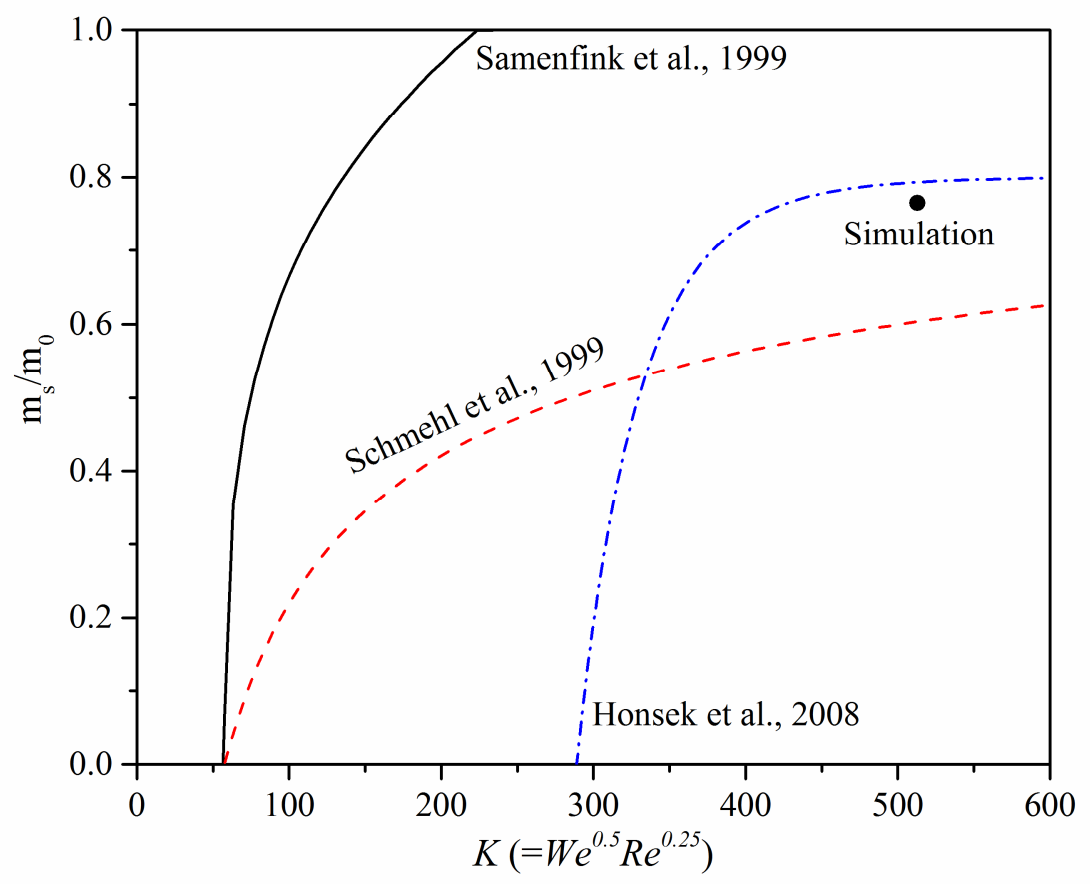

Figure 26. Comparison of mass loss from splashing with empirical models.

Figure 27 shows the simulation result at the ambient air density of $1.225 \mathrm{~kg} / \mathrm{m}^{3}$ in $3 \mathrm{D}$ coordinate system. The $3 \mathrm{D}$ simulation captures the formation and propagation of the lamellae, which is consistent with the $2 \mathrm{D}$ axisymmetric result. For $3 \mathrm{D}$ simulation, at the top of the lamella, the free rim becomes unstable and breaks up into multiple secondary droplets. 


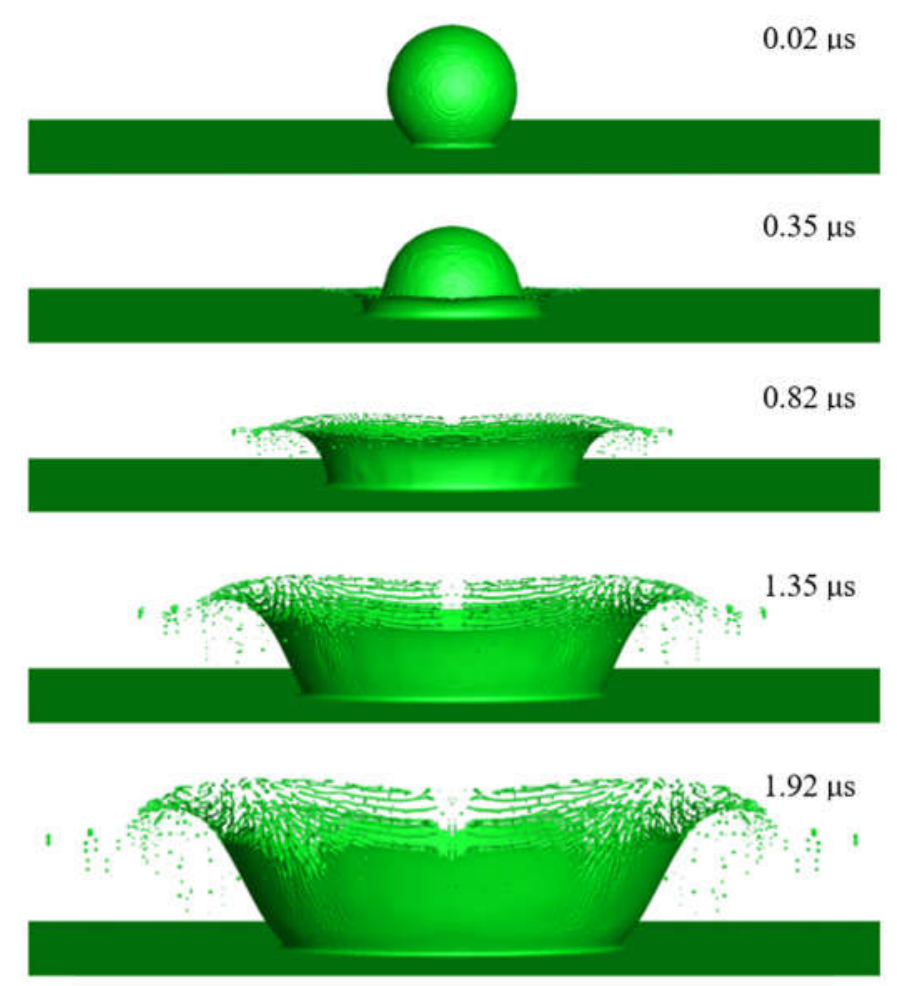

Figure 27. 3D simulation of droplet impact on thin liquid film.

\subsection{Conclusions}

Numerical simulations were conducted to understand the effect of ambient air on drop splashing. The Navier-Stokes equations were solved using the projection method on Cartesian grids. The moment of fluid method was used to construct the interface. A curvature based adaptive method was used to refine meshes near the interface with high curvature. Our simulation showed that MOF was able to accurately capture the interface. We also showed that the dynamic contact angle model gave better prediction than the static contact angle model. Our simulation showed that the splash was attenuated when the air density was lowered. Our simulation revealed that a thin film was formed after the droplet 
contacted the solid surface. The thin film moved at a low speed at the contact with the solid due to viscous effect while the film moved at a high speed away from the solid. The splashing is due to the aerodynamic forces on the thin film. For thin film splashing, the lamella is initiated from the preexisting liquid film on solid surface and the liquid content from the droplet sliding along above it. The thin film splashing is found to be not affected by the ambient air prominently but has much larger mass loss compared with dry surface splashing. 


\section{CHAPTER 4. HIGH-SPEED OBLIQUE DROP IMPACT ON THIN LIQUID FILMS ${ }^{2}$}

\subsection{Introduction}

The phenomenon of drop impingement on a wall has attracted much attention due to its various practical applications such as spray cooling, inkjet printing, and pesticide spraying. Although low-speed normal impacts are involved in most of these applications, high-speed oblique drop impact is critical for the studies of aircraft icing, fuel injection in internal combustion engines, steam turbines, etc. Many experimental and numerical studies (Ching et al., 1984; Rein, 1993; Mundo et al., 1995; Yarin \& Weiss, 1995; Cossali et al., 1997; Samenfink et al., 1999; Weiss \& Yarin, 1999; Roisman \& Tropea, 2002; Thoroddsen, 2002; Josserand \& Zaleski, 2003; Rioboo et al., 2003; Cossali et al., 2004; Leneweit et al., 2005; Nikolopoulos et al., 2005; Xu et al., 2005; Yarin, 2006; Mukherjee \& Abraham, 2007; Nikolopoulos et al., 2007; Deegan et al., 2008; Okawa et al., 2008; Motzkus et al., 2009; Mani et al., 2010; Schroll et al., 2010; Latka et al., 2012; Mandre \& Brenner, 2012; Thoraval et al., 2012; Zhang et al., 2012; Tran et al., 2013; Shetabivash et al., 2014; Guo

2 This chapter appeared in the following publication:

Yisen Guo and Yongsheng Lian, "High-Speed Oblique Drop Impact on Thin Liquid Films," Phys. Fluids 29, 082108 (2017).

(AIP Publishing permits authors to include their published articles in a thesis or dissertation. Formal permission from AIP Publishing is not needed. (https://publishing.aip.org/authors/copyright-reuse)) 
et al., 2016; Josserand \& Thoroddsen, 2016) have been conducted to understand drop impact phenomenon. These studies revealed that the morphologies and underlying mechanisms of dry surface impact and wet surface impact are fundamentally different (Yarin, 2006; Guo et al., 2016).

When a drop impacts on a dry surface, the drop can deposit, rebound or splash, depending on parameters such as drop size, impact velocity, surface tension, substrate properties, and surrounding gas density (Rein, 1993; Yarin, 2006; Josserand \& Thoroddsen, 2016). Mundo et al. (Mundo et al., 1995) experimentally showed that there are two possible outcomes from the impingement of drops on dry solid surfaces: less energetic drops deposit on the surface and form a liquid film, while energetic drops splash and form secondary drops. The authors characterized the transition from deposition to splashing using the parameter $K=$ $R e^{0.25} \cdot W e^{0.5}$ where the splashing threshold is 57.7. The Reynolds number and the Weber number are defined as

$$
R e=\frac{\rho D_{0} V_{0}}{\mu}, \quad W e=\frac{\rho D_{0} V_{0}^{2}}{\sigma},
$$

where $\rho, \mu$, and $\sigma$ are the liquid density, viscosity, and surface tension, respectively, and $D_{0}$ and $V_{0}$ are the drop diameter and impact velocity, respectively. Experimental studies by Xu et al. (Xu et al., 2005) and Latka et al. (Latka et al., 2012) found that air plays a crucial role in determining the dry surface splashing behavior as a low pressure can effectively suppress the splashing. Guo et al. (Guo et al., 2016) numerically investigated the role of ambient air in drop splashing on dry and wet surfaces, confirming that the splashing can be suppressed when the ambient air effects are negligible and the thin film splashing is not affected by the ambient air prominently. 
Under most practical circumstances, drop impact on dry surface is less common. Instead, surface is often wet from previous drop impingements, and hence drop often impacts on surface covered by a liquid layer. When a drop impacts on a liquid layer or wet surface, splashing is generally observed and coalescence occurs only when the drop momentum is low (Rein, 1993; Yarin, 2006). The liquid layer can be described as a film or pool depending on the non-dimensional parameter $\delta$, defined as $h_{0} / D_{0}$, where $h_{0}$ is the liquid layer thickness and $D_{0}$ is the drop diameter. When $\delta$ is less than 1 , it is referred to as liquid film (Cossali et al., 1997; Motzkus et al., 2009).

Experimental studies of thin liquid film impact (Roisman \& Tropea, 2002; Josserand \& Zaleski, 2003; Deegan et al., 2008) classified different regimes of motion after impact, depending on the Reynolds number, the Weber number, and the ratio of film thickness to drop diameter. For very low impact velocity, the drop deposits onto the thin liquid film without crown formation. For high impact velocity, spreading occurs if a radially expanding crown is observed but does not break up into smaller secondary drops. When the $K$ number is greater than a threshold, splashing occurs through a two-stage process: in the first stage a horn-shaped jet is formed from the neck region between the drop and liquid layer and the jet develops to form a crown shape; in the second stage the crown tip breaks up to secondary drops due to instability.

Various models based on experiments have been proposed to predict splash threshold for drop impact on liquid layer. Table 2 lists several commonly used models (Yarin \& Weiss, 1995; Cossali et al., 1997; Samenfink et al., 1999; Deegan et al., 2008). Figure 28 compares these splash threshold models for water drops impact on thin films of $\delta=0.1$. The model of Yarin and Weiss (Yarin \& Weiss, 1995) predicts the highest threshold impact velocity 
for splashing among all and the difference in the predicted threshold velocities is larger for smaller drops.

Table 2. Selected splash threshold models for drop impact on liquid film

\begin{tabular}{lc}
\hline & Splash threshold \\
\hline $\begin{array}{l}\text { Yarin and Weiss (Yarin \& } \\
\text { Weiss, 1995) }\end{array}$ & $V_{0}^{5 / 8} D_{0}^{3 / 8} \rho^{3 / 8} \sigma^{-1 / 4} \mu^{-1 / 8}>17$ \\
Cossali et al. (Cossali et al., & $R e^{0.4} W e^{0.8}>2100+5880 \delta^{1.44}$ for $0.1<\delta<1$ \\
$\begin{array}{l}\text { 1997) } \\
\text { Samenfink et al. (Samenfink et }\end{array}$ & $R e^{0.162} \cdot W e^{0.419}>24$ \\
al., 1999) & $R e^{0.5} \cdot W e>26000$ \\
$\begin{array}{l}\text { Deegan et al. (Deegan et al., } \\
\text { 2008) }\end{array}$ & \\
\hline
\end{tabular}

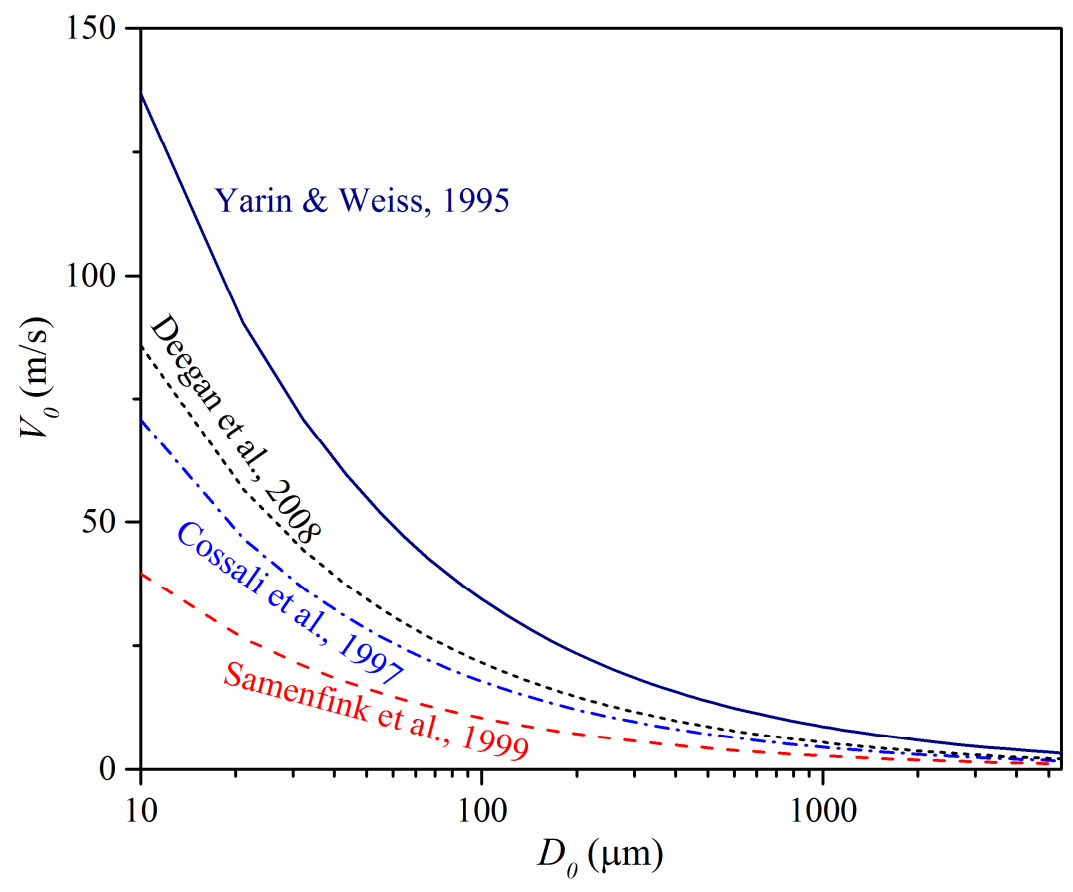

Figure 28. Selected splash threshold models for water drops impact on liquid films of $\delta=$ 0.1 . The abscissa represents drop diameter and the ordinate represents the corresponding threshold velocity for splash. 
Other studies have been conducted to investigate the jet formation and the secondary drop formation. Weiss and Yarin (Weiss \& Yarin, 1999) simulated drop impact on a liquid film to study the formation of jet in the neck region between the drop and liquid film after impact. For sufficiently energetic impacts, the authors found that the jet can pinch off a torus-shaped liquid volume at its tip or reconnect with the liquid film. Thoroddsen (Thoroddsen, 2002) experimentally verified jet formation in the neck region between drop and liquid film. The mechanism of secondary drop formation was also investigated (Allen, 1975; Fullana \& Zaleski, 1999; Rieber \& Frohn, 1999; Roisman et al., 2006; Nikolopoulos et al., 2007; Krechetnikov \& Homsy, 2009). Different mechanisms were used to explain the crown formation instability in splashing, including the Rayleigh-Taylor instability (Allen, 1975), Rayleigh instability (Rieber \& Frohn, 1999), Plateau-Rayleigh capillary instability (Fullana \& Zaleski, 1999; Roisman et al., 2006; Nikolopoulos et al., 2007), and Richtmyer-Meshkov instability (Krechetnikov \& Homsy, 2009).

Most previous studies focused on normal impact with relatively low impact velocity $(<30$ $\mathrm{m} / \mathrm{s}$ ) and film thickness, $\delta$, greater than 0.1 (Rein, 1993; Yarin \& Weiss, 1995; Cossali et al., 1997; Samenfink et al., 1999; Weiss \& Yarin, 1999; Thoroddsen, 2002; Rioboo et al., 2003; Cossali et al., 2004; Nikolopoulos et al., 2005; Yarin, 2006; Mukherjee \& Abraham, 2007; Nikolopoulos et al., 2007; Motzkus et al., 2009; Shetabivash et al., 2014). Study of high-speed oblique impact on thin liquid film is rare. Among the few oblique drop impact studies, experiments by Ching et al. (Ching et al., 1984), Leneweit et al. (Leneweit et al., 2005) and Zhbankova and Kolpakov (Zhbankova \& Kolpakov, 1999) investigated oblique impact on liquid pool $(\delta>1)$ in the non-splash regime. Okawa et al (Okawa et al., 2008) experimentally studied the effect of impact angle on the total mass of secondary drops 
produced during the collision. Liang et al. (Liang et al., 2013) experimentally studied the effects of surface tension and viscosity on spreading and splashing behaviors. Cheng and Lou (Cheng \& Lou, 2015) numerically studied drop oblique impact $\left(30^{\circ} \leq \theta \leq 90^{\circ}\right)$ on a wet wall using the Lattice-Boltzmann model and observed a transition from crown splashing to single-sided splashing, however, their model artificially introduced to a cosine-wave perturbation on the surface of drop before impact to form splashing.

In the present study, we numerically investigate the high-speed drop impacts on thin liquid films. The paper is structured as follows. First, we present the numerical method. Second, we validate our code with experiment and theoretical solution. Subsequently, we investigate normal and oblique impacts of water drops on water films of different impact angles and film thicknesses. Finally, we study the normal impacts of different film-drop density ratios.

\subsection{Results and Discussions}

We first validate the code by comparing with experimental results and theoretical predictions using three cases. Then we investigate the effects of impact angle and film thickness by simulating the oblique impacts of $41.3-\mu \mathrm{m}$-diameter water drops on water films of different thicknesses at different impact angles. The film thicknesses used in the simulations are $1 \mu \mathrm{m}, 5 \mu \mathrm{m}$ and $20 \mu \mathrm{m}$ and the impact angles are $60^{\circ}, 45^{\circ}, 30^{\circ}$ and $15^{\circ}$. Finally, we present the simulation results of the impacts of different film-drop density ratios. Table 3 summarizes the parameters for all simulation cases. 
Table 3. Parameters used in numerical simulations

\begin{tabular}{|c|c|c|c|c|c|c|c|c|c|c|}
\hline & & \multirow{2}{*}{$\begin{array}{c}\text { Drop } \\
\text { diameter } \\
D_{0}\end{array}$} & \multirow{2}{*}{ Liquid type } & \multicolumn{2}{|c|}{$\begin{array}{c}\text { Impact velocity } V_{0} \\
(\mathrm{~m} / \mathrm{s})\end{array}$} & \multirow{2}{*}{$\begin{array}{l}\text { Impact } \\
\text { angle } \theta\end{array}$} & \multirow{2}{*}{$\begin{array}{c}\text { Film } \\
\text { thickness } \\
h_{0}\end{array}$} & \multirow{2}{*}{$\begin{array}{c}\delta= \\
h_{0} / D_{0}\end{array}$} & \multirow{2}{*}{$W e$} & \multirow{2}{*}{$R e$} \\
\hline & & & & $\begin{array}{c}\text { Normal } \\
V_{0 n} \\
\end{array}$ & $\begin{array}{c}\text { Tangential } \\
V_{0 t} \\
\end{array}$ & & & & & \\
\hline \multirow{5}{*}{$\begin{array}{l}\text { A. Code } \\
\text { validation }\end{array}$} & \multirow{2}{*}{ Case 1} & \multirow{2}{*}{$\begin{array}{l}3.82 \\
\mathrm{~mm}\end{array}$} & \multirow{2}{*}{ water } & 3.94 & \multirow{2}{*}{0} & \multirow{2}{*}{$\begin{array}{c}90^{\circ} \\
\text { (normal) }\end{array}$} & \multirow{2}{*}{$2.57 \mathrm{~mm}$} & \multirow{2}{*}{0.67} & 842 & 15,366 \\
\hline & & & & 3.51 & & & & & 667 & 13,676 \\
\hline & Case 2 & $2 \mathrm{~mm}$ & silicone oil & 1.83 & 0 & $\begin{array}{c}90^{\circ} \\
\text { (normal) }\end{array}$ & $0.4 \mathrm{~mm}$ & 0.2 & 324 & 2191 \\
\hline & \multirow{2}{*}{ Case 3} & \multirow{2}{*}{$41.3 \mu \mathrm{m}$} & \multirow{2}{*}{ water } & \multirow{2}{*}{53} & \multirow{2}{*}{0} & \multirow{2}{*}{$\begin{array}{c}90^{\circ} \\
\text { (normal) }\end{array}$} & $1 \mu \mathrm{m}$ & 0.024 & \multirow{2}{*}{1590} & \multirow{2}{*}{1680} \\
\hline & & & & & & & $5 \mu \mathrm{m}$ & 0.12 & & \\
\hline \multirow{3}{*}{\multicolumn{2}{|c|}{$\begin{array}{l}\text { B. Effect of impact } \\
\text { angle }\end{array}$}} & \multirow{3}{*}{$41.3 \mu \mathrm{m}$} & \multirow{3}{*}{ water } & \multirow{3}{*}{53} & 30.6 & $60^{\circ}$ & \multirow{3}{*}{$5 \mu \mathrm{m}$} & \multirow{3}{*}{0.12} & 2121 & 1940 \\
\hline & & & & & 91.8 & $30^{\circ}$ & & & 6362 & 3361 \\
\hline & & & & & 197.8 & $15^{\circ}$ & & & 23,742 & 6493 \\
\hline \multirow{3}{*}{\multicolumn{2}{|c|}{$\begin{array}{l}\text { C. Effect of film } \\
\text { thickness }\end{array}$}} & \multirow{3}{*}{$41.3 \mu \mathrm{m}$} & \multirow{3}{*}{ water } & \multirow{3}{*}{53} & \multirow{3}{*}{53} & \multirow{3}{*}{$45^{\circ}$} & $1 \mu \mathrm{m}$ & 0.024 & \multirow{3}{*}{3185} & \\
\hline & & & & & & & $5 \mu \mathrm{m}$ & 0.12 & & 2378 \\
\hline & & & & & & & $20 \mu \mathrm{m}$ & 0.48 & & \\
\hline $\begin{array}{l}\text { D. Effect o } \\
\text { ratio }\end{array}$ & density & $41.3 \mu \mathrm{m}$ & $\begin{array}{l}\frac{\rho_{\text {film }}}{\rho_{\text {water }}} \\
=0.5,1,1.5\end{array}$ & 53 & 0 & $\begin{array}{c}90^{\circ} \\
\text { (normal) }\end{array}$ & $5 \mu \mathrm{m}$ & 0.12 & 1590 & 1680 \\
\hline
\end{tabular}

A schematic of the simulation setup of a liquid drop impacting onto thin liquid film is shown in Figure 29. The liquid drop diameter is $D_{0}$ and the liquid film thickness is $h_{0}$. The drop is surrounded by air above the liquid film. The impact angle is $\theta$ and the impact velocity is $V_{0}$, and $V_{0 t}$ and $V_{0 n}$ are the tangential and normal components of impact velocity respectively. The dimensionless time is defined as $T=\frac{V_{0 n} \cdot t}{D_{0}}$, and the dimensionless film thickness is defined as $\delta=\frac{h_{0}}{D_{0}}$. Due to symmetry of the problem, the computational domain of a three-dimensional model only contains one half of the drop for oblique impact and one quarter of the drop for normal impact. Although three-dimensional model can capture ejected secondary drops and crown breakup, these phenomena are not the main focus of this study. Therefore, two-dimensional numerical model is also used to investigate flow 
behaviors with higher grid resolution. The tangential velocity component points to the right in Figure 29 and we define the right side of the drop as upstream side and left side as downstream side.

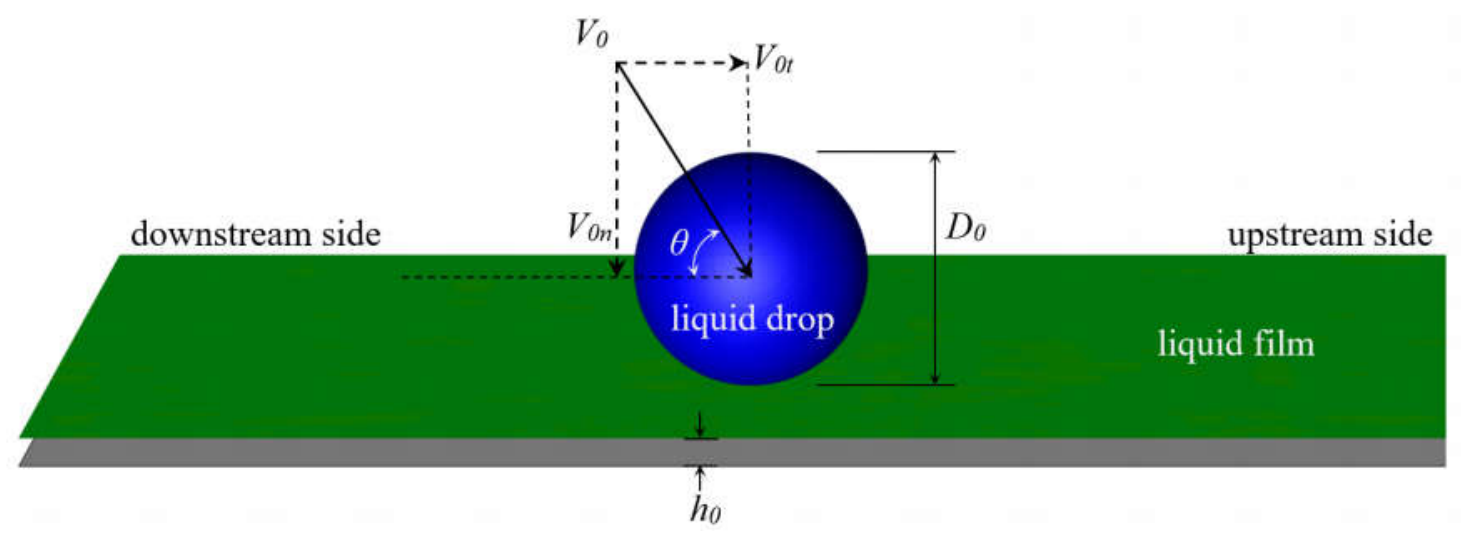

Figure 29. Illustration of the simulation setup of a liquid drop impacting onto thin liquid film.

\subsubsection{Code validation}

Three code validation study cases are performed and presented in this section. The three cases represent low-speed impact on thick liquid film, low-speed impact on thin liquid film, and high-speed impact on thin liquid film, respectively.

\subsubsection{Validation case 1}

We first validate the code with experiment of normal impact on thick liquid film. The experiment was conducted by Cossali et al. (Cossali et al., 2004). The water drop diameter was $3.82 \mathrm{~mm}$, the terminal impact velocity was $3.94 \mathrm{~m} / \mathrm{s}$, the film thickness was $2.57 \mathrm{~mm}$, and the resulting $W e$ was 842 and $R e$ was 15,366 . Our simulation has the same initial conditions as the experiment setup. A grid convergence study is performed using different 
effective grid resolutions defined by the number of cells per initial diameter of the drop. As shown in Figure 30, the effective grid resolutions of 40, 52, 62 and 74 cells per diameter (cpd) are used to measure the convergence of crown radius $\left(r_{C}\right)$ at $T=1 . T$ is the dimensionless time which is defined as $T=\frac{V_{0 n} \cdot t}{D_{0}}$. The crown radius only increases by 1.7 $\%$ when the grid resolution is increased from $62 \mathrm{cpd}$ to $74 \mathrm{cpd}$, but the total CPU time to finish simulation to $T=4$ almost doubled. To keep computational cost relatively low with reasonable simulation accuracy, the grid resolution of $62 \mathrm{cpd}$ is adopted in this case. Similar grid convergence study is carried out for all simulation cases in this paper.

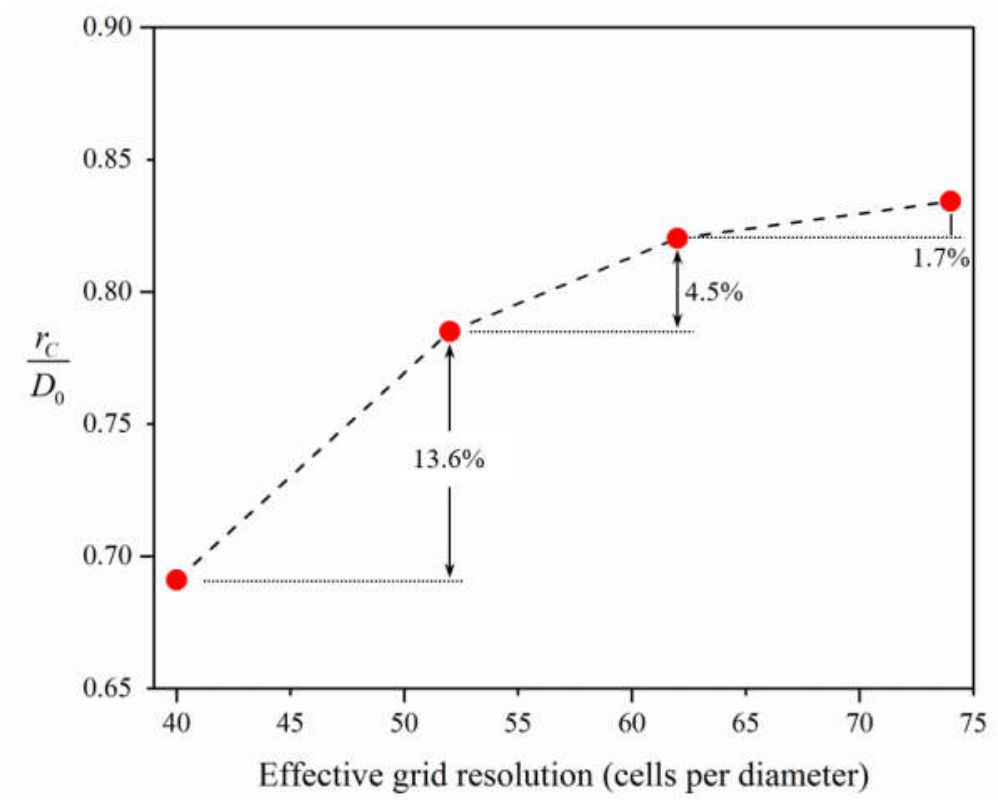

Figure 30. Convergence of crown radius at $T=1$. The effective grid resolutions are 40, 52, 62 and 74 cells per diameter. The percentages show the dimensionless crown radius increase when increasing grid resolution. 
Figure 31 compares our simulation result with the experiment. Only a quarter of the domain is simulated and the simulation result is mirrored to show the full drop impact. As shown in Figure 31, our simulation matches the experimental result to capture the jets ejected from the crown and the formation of secondary drops. The experiment shows ripples inside the crown, which is possibly caused by the shape oscillation of the drop. Because no such perturbation exists in the simulation, the ripple effect is not observed.

To better illustrate the drop impact phenomenon, we use different materials to represent the liquid drop and liquid film even though both have the same properties of water. Throughout this paper we use the following color code to represent different materials in three-dimensional simulations: white for air, gray for solid substrate, green for liquid film, and blue for liquid drop. Simulating drop and liquid film in different materials allows for the mass originating in the drop and the liquid film to be tracked through the impact process. Our numerical simulation shows that the drop mainly embeds inside the crown and most liquid of the crown is from liquid film. Rim of the crown and secondary drops have mixed color of green and blue, indicating they are mixtures from the drop and the liquid film. 
(a)

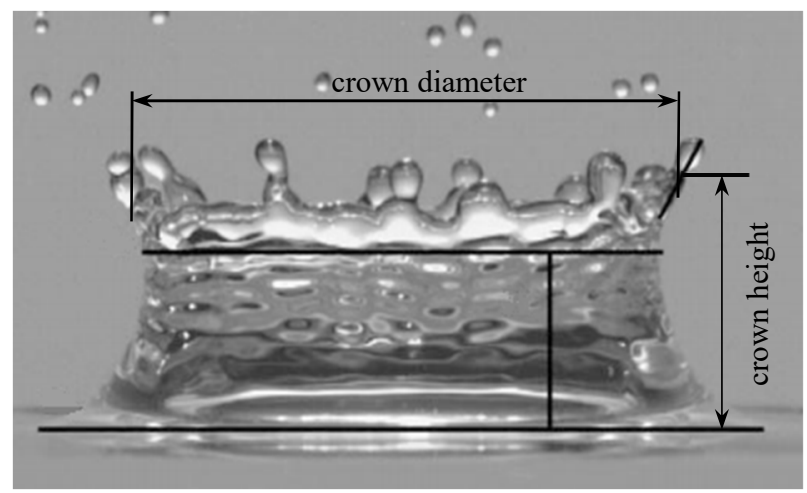

(b)

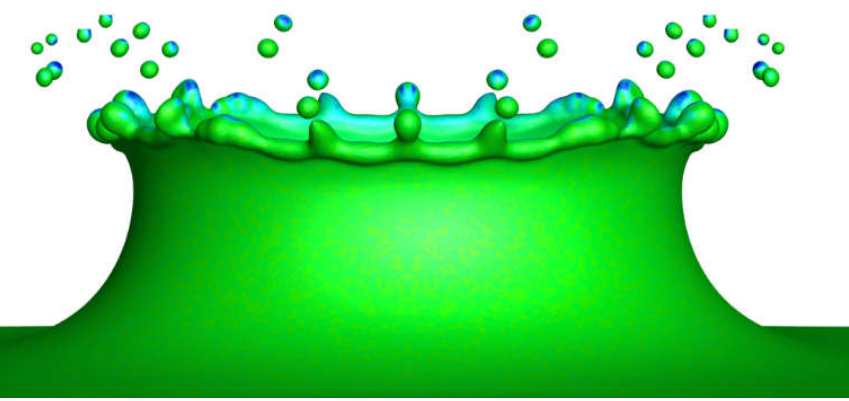

Figure 31. Water drop normal impact onto a $2.57 \mathrm{~mm}$ thick water film with the drop dimeter of $3.82 \mathrm{~mm}$ and terminal impact velocity of $3.94 \mathrm{~m} / \mathrm{s}$ ( We $=842$ and $R e=15,366)$. (a): experiment (Cossali et al., 2004); (b): simulation.

We validate our code quantitatively against experimental measurement by Cossali et al. (Cossali et al., 2004) of a 3.82-mm-diameter water drop impact onto a 2.57 -mm-thick water film with the impact velocity of $3.51 \mathrm{~m} / \mathrm{s}(W e=667, R e=13,676$, and $\delta=0.67)$. Cossali et al. measured the time evolution of the outer diameter of the formed crown. As shown in Figure 31(a), the crown diameter is measured from base of the ejected jets. Our simulated time evolution of the crown diameter is compared with experimental results in Figure 32. The rim of the crown moves relatively fast outward at the beginning at a speed of about 3 $\mathrm{m} / \mathrm{s}$ and then it slows down to about $0.5 \mathrm{~m} / \mathrm{s}$ at the end of the simulation. The simulation shows good agreement with the experimental result. 
In the code validation with low-speed drop impact on thick liquid film, our simulation tool shows good agreement with experiments qualitatively and quantitatively. The crown shape is similar to experimental observation and the time evolutions of crown are in agreement.

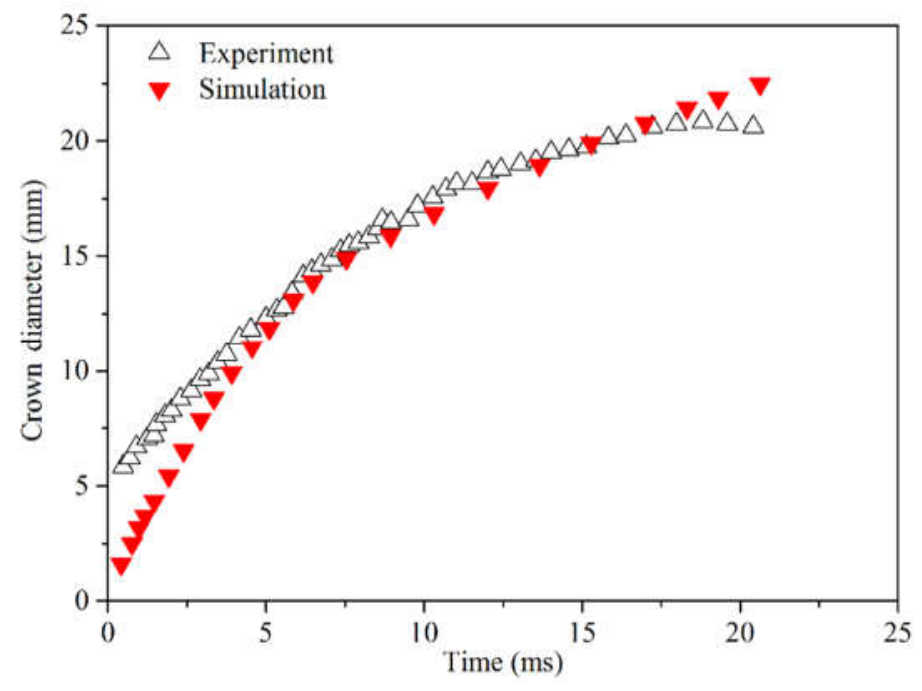

Figure 32. Time evolutions of crown diameters of the impact of a 3.82-mm-diameter water drop onto a $2.57-\mathrm{mm}$-thick water film with the impact velocity of $3.51 \mathrm{~m} / \mathrm{s}(\mathrm{We}=667, \mathrm{Re}$ $=13,676$, and $\delta=0.67)$. The simulation result $(\nabla)$ shows good agreement with the experimental measurement $(\triangle)($ Cossali et al., 2004).

\subsubsection{Validation case 2}

In the second validation case, we compare simulation result with experiment of low-speed impact on thin liquid film. The experiment conducted by Zhang et al. (Zhang et al., 2012) studied a 2-mm-diameter silicon oil impact on the same liquid film with $W e=324, R e=$ 2191 and $\delta=0.2$. The simulation of a 2-mm-diameter silicon oil drop impact on a $0.4-\mathrm{mm}$ thick same liquid film with the impact velocity of $1.83 \mathrm{~m} / \mathrm{s}$ is conducted, which leads to the same $W e$ and $R e$. The physical properties of the liquid in the simulation is the same as 
in the experiment. We use two-dimensional axisymmetric coordinates in the simulation because secondary drops ejection or crown breakup is not observed in the experiment. The effective grid resolution is $780 \mathrm{cpd}$. Figure 33(a) shows the simulation result with the drop and the liquid film distinguished in different colors. The air bubbles trapped under the drop is shown is blue color, and similar air entrainment phenomenon is observed in experiments (Tran et al., 2013). Figure 33(b) compares the liquid surface profiles between the experimental image and numerical simulation at $t=0.335 \mu$ s and $0.331 \mu \mathrm{s}$, respectively. The comparison shows our simulation is in good agreement with experiment.
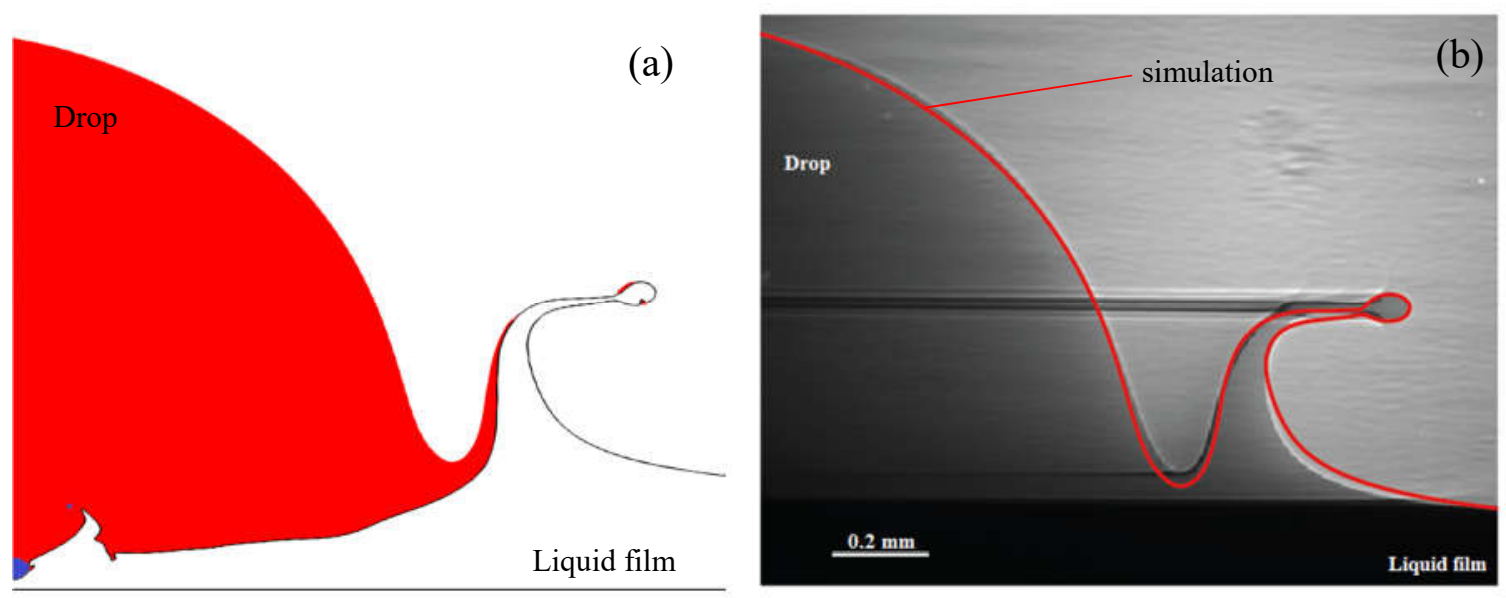

Figure 33. Validation with experiment by Zhang et al. (Zhang et al., 2012). $W e=324, R e$ $=2191$ and $\delta=0.2$. The experimental image and our numerical simulation are taken at $t=$ $0.335 \mu \mathrm{s}$ and $0.331 \mu \mathrm{s}$, respectively. (a): numerical simulation showing drop in red color, liquid film interface in black line, and trapped air bubbles in blue color. (b): liquid surface profile comparison of experiment and simulation (red line). 


\subsubsection{Validation case 3}

The third case validates the code for high-speed normal impact of drop on thin liquid film. The water drop has a dimeter of $41.3-\mu \mathrm{m}$ and two thin film thicknesses, 1 and $5 \mu \mathrm{m}(\delta=$ 0.024 and 0.12 ), are used. The normal impact velocity is $53 \mathrm{~m} / \mathrm{s}$. Only a quarter of the drop is simulated due to symmetry of the problem. A grid convergence study is first carried out and the grid resolution of 46 cells per drop diameter shows good convergence of crown radius.

The simulation results are shown in Figure 34. In both cases, the drop completely repels the thin film from the substrate after contact. The drop liquid is pushed radially outward, which is resisted by adjacent film liquid at rest. Following the direction of the least resistant, a thin sheet of liquid is propelled upward to form a crown from the neck region connecting the two liquids. When the drop keeps pushing the thin crown outward, the crown is radially stretched and eventually breaks up into secondary drops. Based on their experiments, Rioboo et al. (Rioboo et al., 2003) observed that when the dimensionless film thickness, $\delta$, is smaller than 0.03 , the drop splashes without crown formation. Nevertheless, Figure 34 clearly demonstrates the crown formation for $\delta=0.024$. One possible reason is that the experiments of Rioboo et al. (Rioboo et al., 2003) were conducted at low velocities $\left(V_{0}<3.14 \mathrm{~m} / \mathrm{s}\right)$, their observation may not be valid for high-speed drop impact. We hypothesize that the high-speed impingement creates a high speed thinner lamella moving outward, leading to higher resistance and upward motion of the lamella.

A close comparison of the two cases in Figure 34 reveals some distinct features. First, the horn-shaped jet formed from the neck region appears earlier for the thinner film case. Second, the thinner film case has earlier crown breakup and more secondary drops. Third, 
the thinner film case has thinner crown which moves faster both outward and upward. The crown motions can be quantitatively measured, as shown in Figure 35, the time evolutions of crown radius and height are plotted. The crown radius, $r_{c}$, and crown height, $h_{c}$, are measured from the base of the ejected jets, as illustrated in Figure 31(a). Comparisons are also made with the theoretical prediction of crown radius change with time. The theoretical prediction is derived by Yarin and Weiss (Yarin \& Weiss, 1995) and is given by

$$
\frac{r_{c}}{D}=\left(\frac{2}{3}\right)^{1 / 4} \frac{V_{0}^{1 / 2}}{D^{1 / 4} h_{0}^{1 / 4}}\left(t-t_{0}\right)^{1 / 2}
$$

where $t_{0}$ is the shifting time and $t_{0}=0.02 \mu$ s. The time evolutions of crown radius from our simulations qualitatively agree with the theoretical predictions. It is also clear from Figure 35 that the thinner film case has larger crown radius and higher crown than the thicker film case. For the thinner film case, the crown moves faster in both outward and upward directions, because thinner crown has less inertia and hence more tendency to be pushed by drop. 

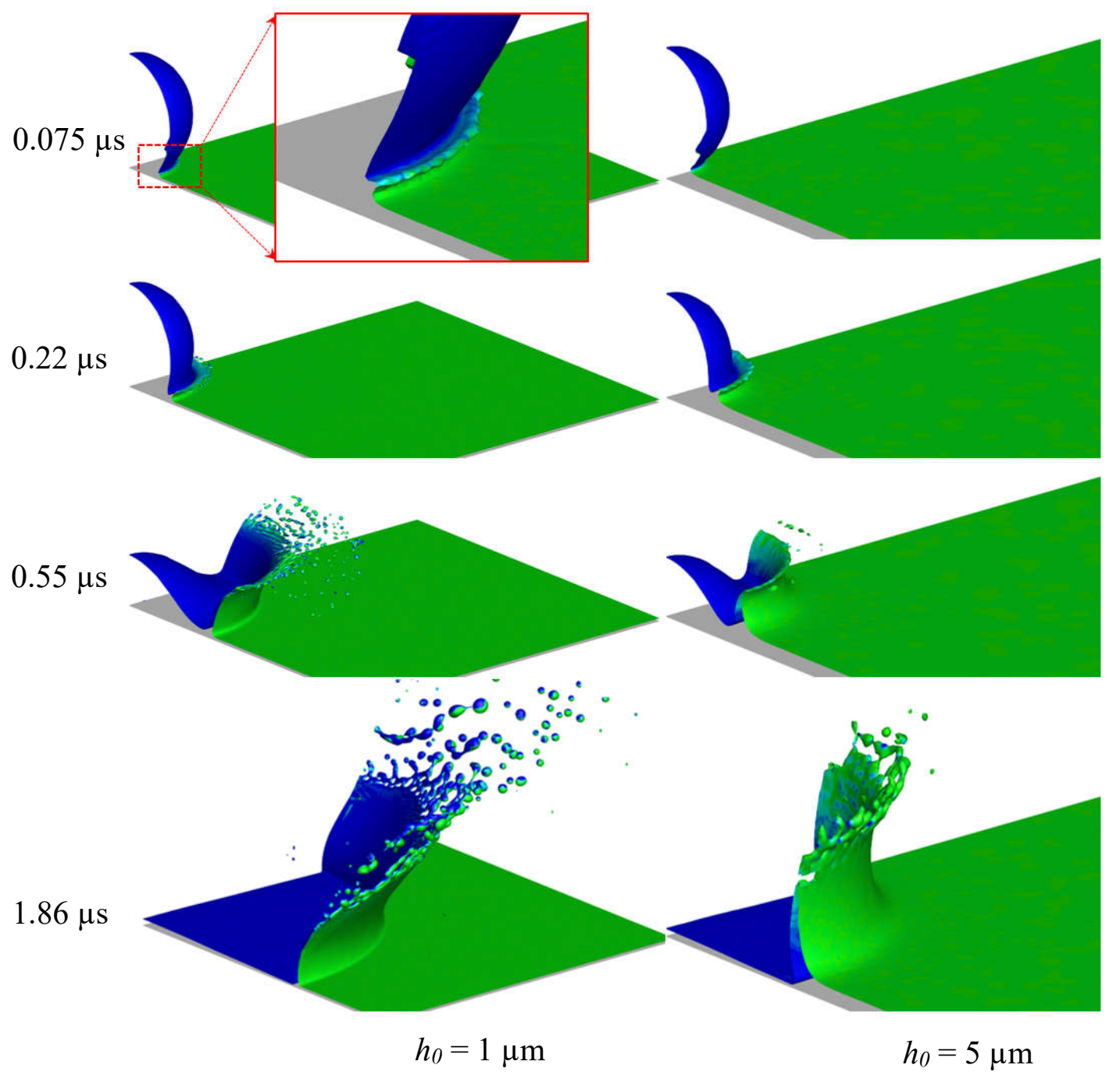

Figure 34. Normal impact of a 41.3- $\mu$ m-diameter water drop on a 1- $\mu$ m-thickness water film (left) and a 5- $\mu \mathrm{m}$-thickness water film (right). Normal impact velocity is $53 \mathrm{~m} / \mathrm{s}$. 

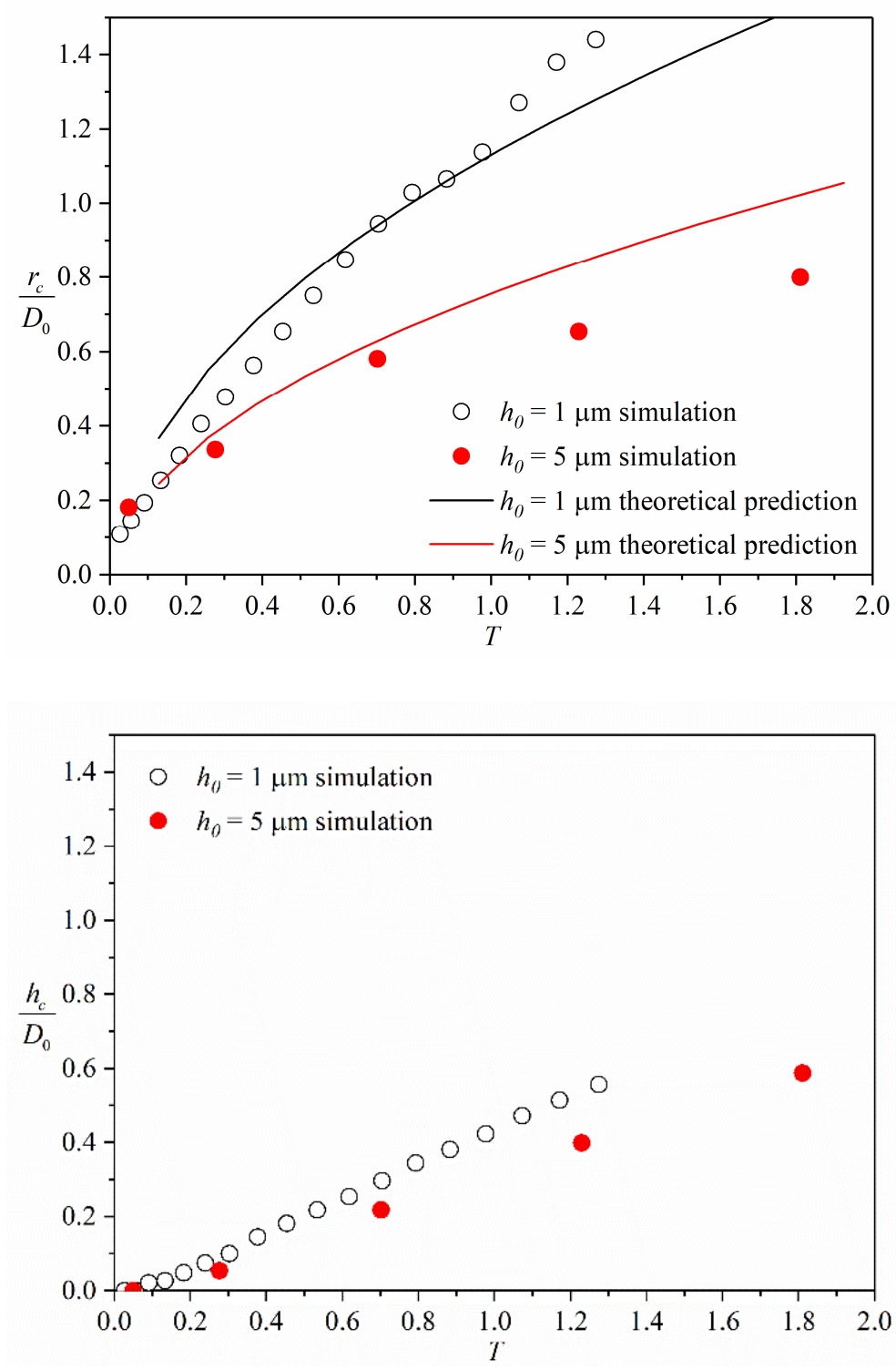

Figure 35. Time evolutions of crown radius, $r_{c}$, and crown height, $h_{c}$. The numerical simulations are shown in Figure 34. $D_{0}=41.3 \mu \mathrm{m}, V_{0}=53 \mathrm{~m} / \mathrm{s}, h_{0}=1 \mu \mathrm{m}$ and $5 \mu \mathrm{m}$.

\subsubsection{Effect of impact angle}

To understand the effect of impact angle on drop impact on liquid film, we maintain the same normal impact velocity but vary the tangential velocity to modify the impact angle. 
Our simulations reveal that smaller impact angle leads to smaller lamella on the downstream side and suppression of lamella development on the upstream side.

In all simulation cases, the water drop dimeter is $41.3 \mu \mathrm{m}$, the film thickness is $5 \mu \mathrm{m}$, and the normal component of impact velocity is $53 \mathrm{~m} / \mathrm{s}$. Two impact angles are first studied: $30^{\circ}$ and $60^{\circ}$. Because the problem is plane-symmetric, only one half of the drop is simulated for three-dimensional numerical model. Snapshots of impact at different time instants are shown in Figure 36. The two cases share similarities. In both cases a vertical liquid lamella is formed on the downstream side. The lamella originates near the intersection between the drop and the liquid film after the impact. Then the lamella moves obliquely upward. The rim of the lamella becomes unstable and breaks up into secondary drops, similar to the normal impact cases shown in Figure 34. The green color of the lamella indicates the liquid is mainly from the pre-existing liquid film.

However, the differences in flow patterns and splashing behaviors are also obvious even though their normal impact velocities are the same. As shown in the $60^{\circ}$ impact angle case (Figure 36 left), crown is formed almost immediately after the impact. Then the drop continues to push the crown lamella moving outward and breaks the crown into secondary drops. During the crown development, the blue color only appears on the inside of the crown lamella, indicating the drop liquid slides on top of the lamella. However, in the $30^{\circ}$ impact angle case (Figure 36 right) completely different behaviors downstream of the drop are shown. No crown is formed on the left of the drop at time instants of $0.60 \mu$ s or 0.81 $\mu$ s. Our simulation shows that the formation of a crown is not encouraged as a result of increasing tangential velocity. Instead, the drop rolls over onto the film liquid at time instants of $0.81 \mu \mathrm{s}$ and $1.25 \mu \mathrm{s}$, as shown in the zoomed-in view of Figure 37 . The rolled- 
up liquid then re-impinges on the quiescent film in front to form splashing. Instead of breaking up into small secondary drops, long liquid fingers are formed by the reimpingement. Both cases also show that the solid surface emerges at the impact site at the later impact stage, as seen at $6.79 \mu \mathrm{s}$, the solid surface at the impact site is almost dry with small amount of drop liquid remains on the solid surface.

As the tangential velocity increases, a larger portion of the drop moves toward upstream side, leaving less liquid drop downstream. Thus, as shown in Figure 38, the crown radius, $r_{c}$, and crown height, $h_{c}$, on the downstream side are both smaller for the $30^{\circ}$ impact angle case than for the $60^{\circ}$ impact angle case. The crown radius, $r_{c}$, and crown height, $h_{c}$, are measured from the base of the ejected jets, as illustrated in Figure 31(a). 

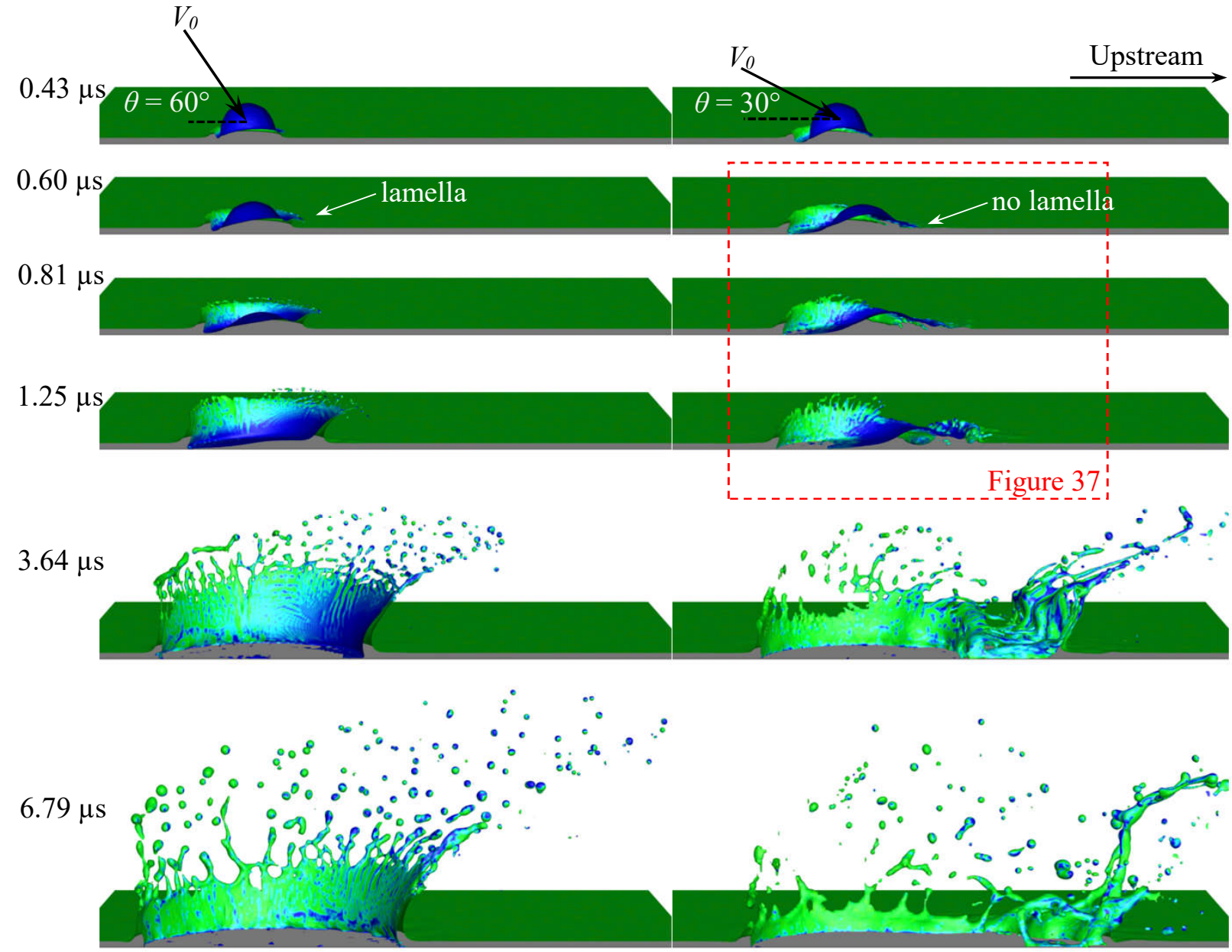

Figure 36. Oblique impact of 41.3- $\mu \mathrm{m}$-diameter water drop on 5- $\mu \mathrm{m}$-thick water film. Left: impact angle is $60^{\circ}\left(V_{0 n}=53 \mathrm{~m} / \mathrm{s}\right.$ and $\left.V_{0 t}=30.6 \mathrm{~m} / \mathrm{s}\right)$. Right: impact angle is $30^{\circ}\left(V_{0 n}=53\right.$ $\mathrm{m} / \mathrm{s}$ and $\left.V_{0 t}=91.8 \mathrm{~m} / \mathrm{s}\right)$. 

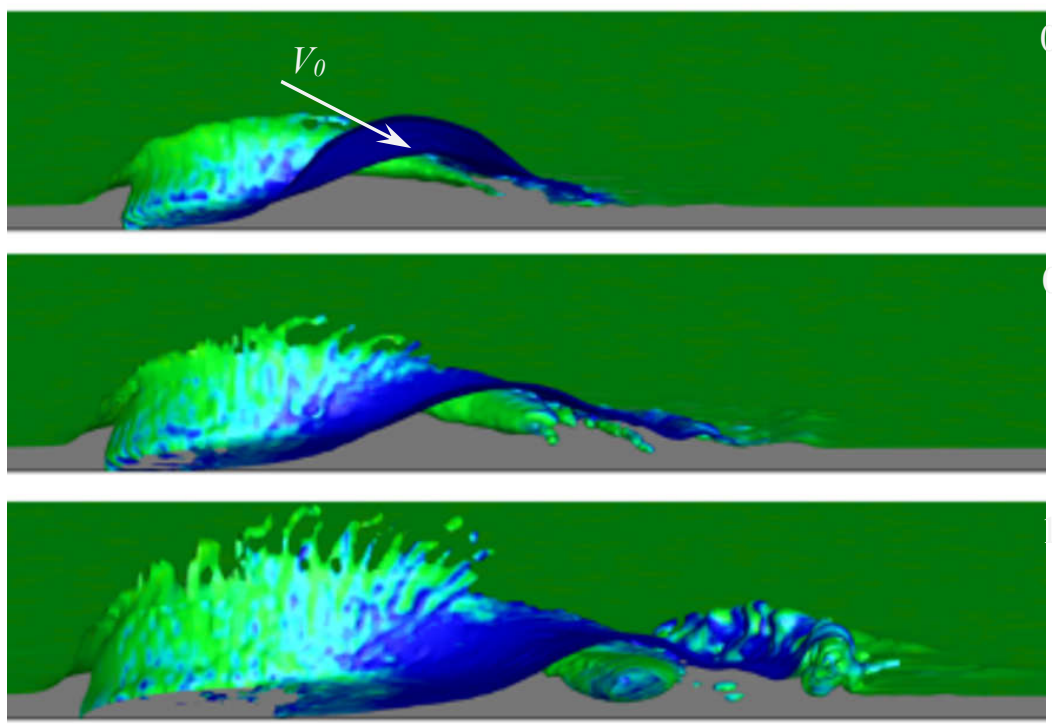

Figure 37. Zoomed-in view of the area bounded by the dashed red rectangle in Figure 36. As the drop impacts onto the film it rolls over the film and then re-impinges on the surrounding quiescent film. 

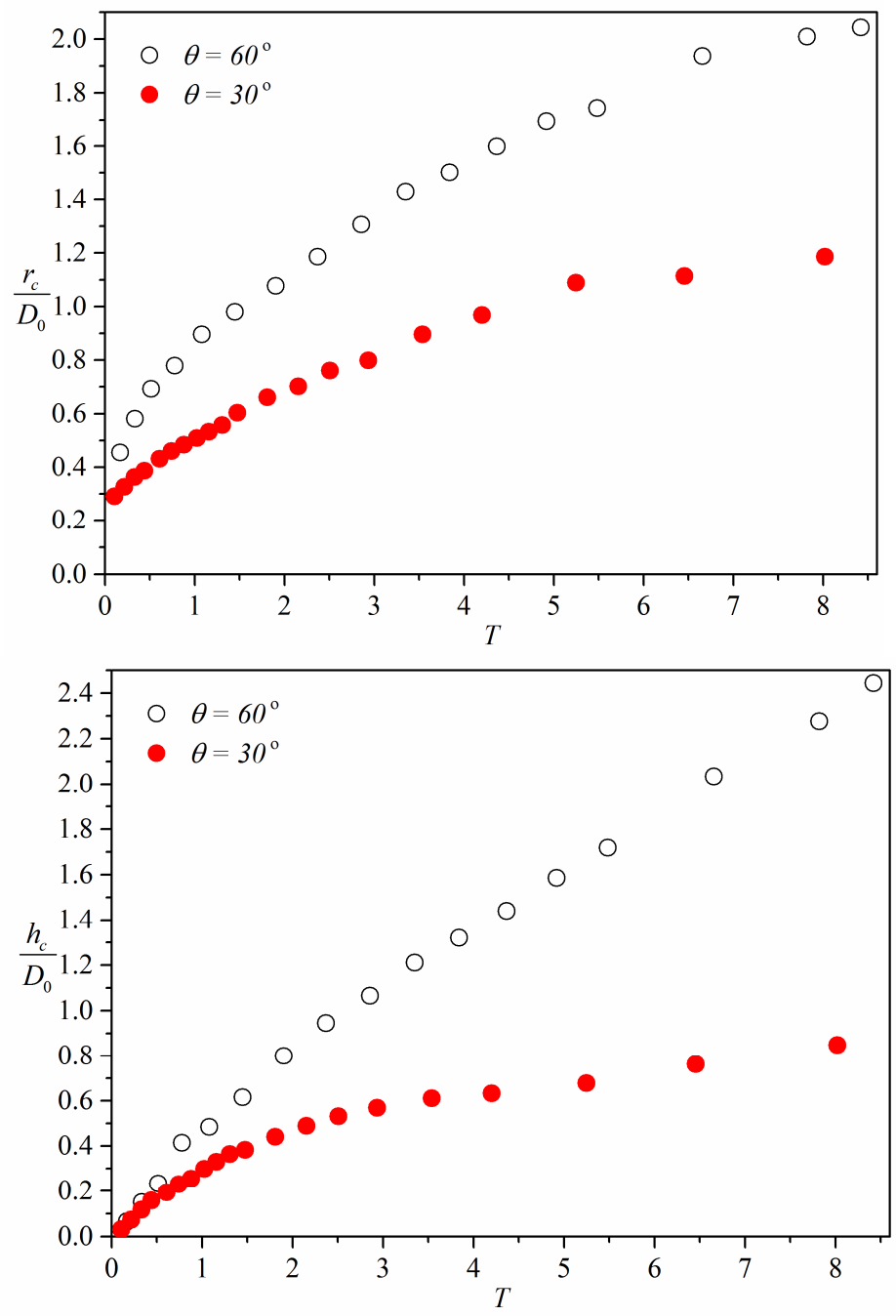

Figure 38. Time evolutions of crown radius, $r_{c}$, and crown height, $h_{c}$, on the downstream side.

To highlight some prominent flow features with higher grid resolution, we simulate the two cases using two-dimensional numerical models. Figure 39 compares the simulation results. The $30^{\circ}$ impact angle case has a higher tangential velocity and the velocity difference across the interface incurs strong Kelvin-Helmholtz instability, which is clearly seen at the interface between the drop and film. Due to perturbation and tangential motion of upper liquid, the interface evolves into an unstable vortex sheet which rolls up into 
spirals. Another difference between the $30^{\circ}$ and $60^{\circ}$ impact angle cases is the liquid volume portions in the expanding lamella at the upstream side. The $30^{\circ}$ impact angle case shows that the liquid from drop suppress the upward movement of liquid film while the $60^{\circ}$ impact angle case shows the two liquids moves outward together. More liquid volume from film is shown in the lamella in the $60^{\circ}$ impact angle case than in the $30^{\circ}$ impact angle case.

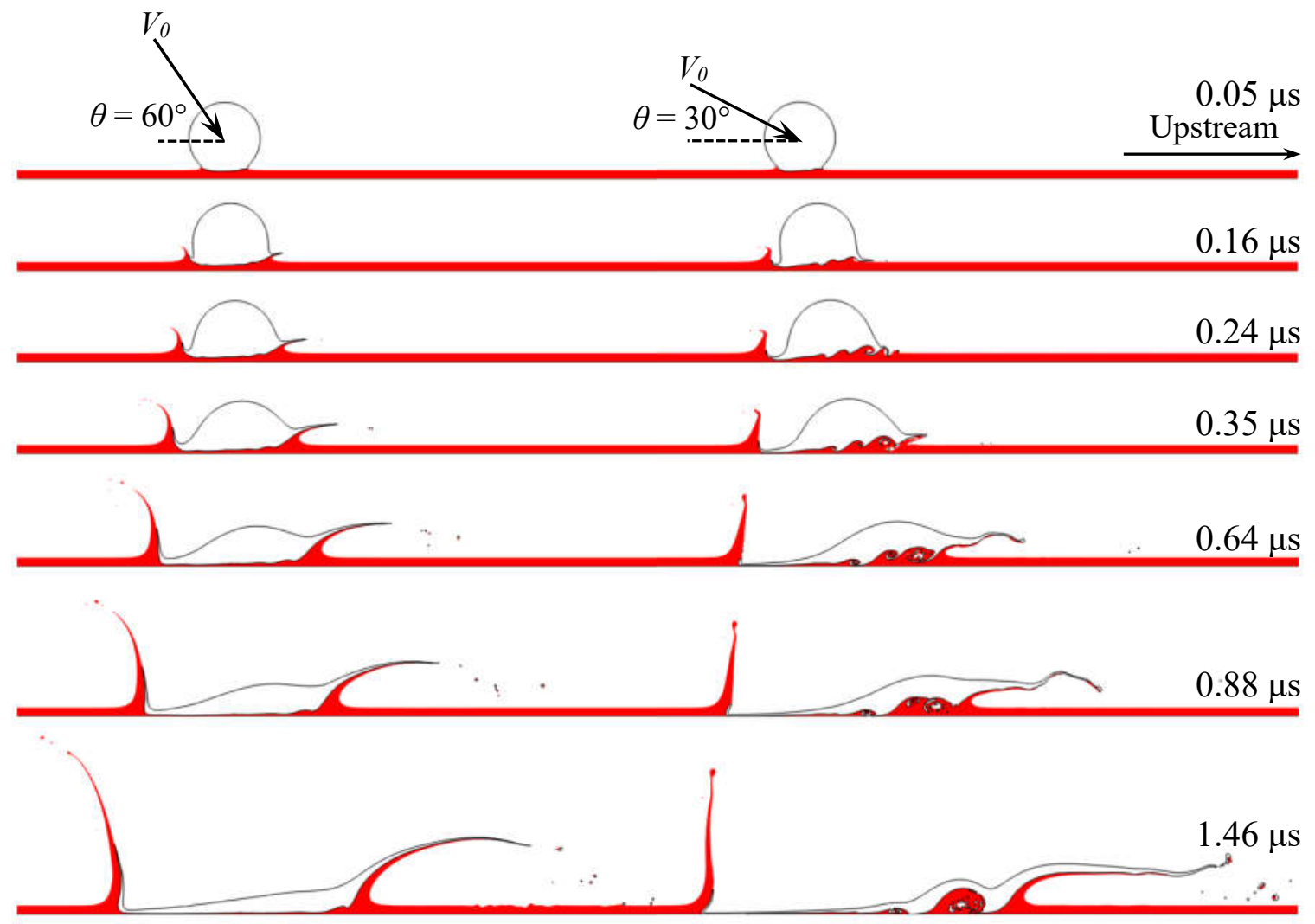

Figure 39. Two-dimensional simulations of oblique impact of a $41.3-\mu \mathrm{m}$-diameter water drop on a $5-\mu \mathrm{m}$-thick water film. White represents drop and red represents film. Left: impact angle is $60^{\circ}$. Right: impact angle is $30^{\circ}$. 
Splashing can be completely suppressed on the upstream side with the increase of tangential impact velocity. As shown in Figure 40, at the $15^{\circ}$ impact angle (tangential impact velocity of $197.8 \mathrm{~m} / \mathrm{s}$ ), no lamella is developed on the upstream side.
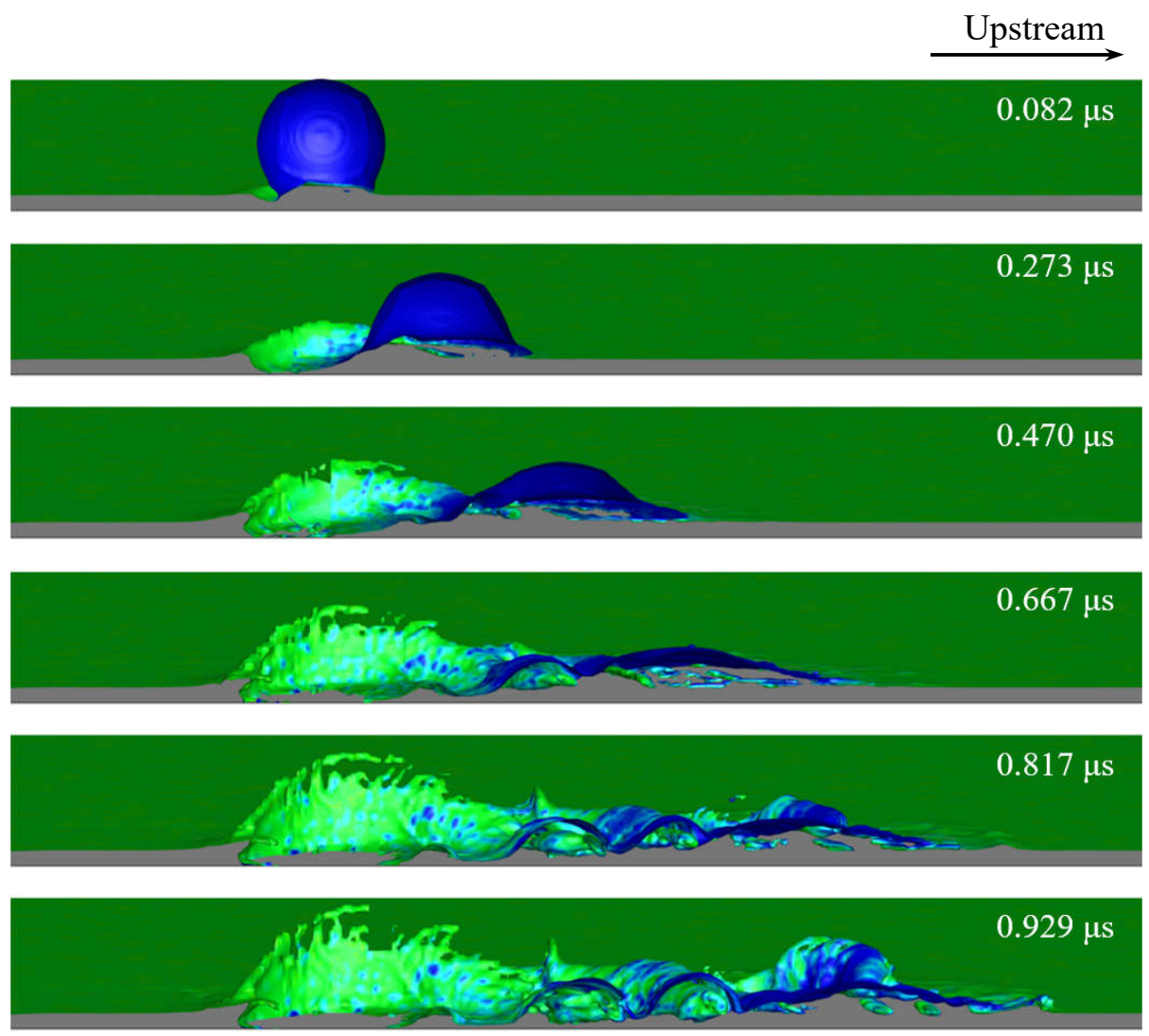

Figure 40. Oblique impact of 41.3- $\mu \mathrm{m}$-diameter water drop on 5 - $\mu \mathrm{m}$-thick water film. Impact angle is $15^{\circ}\left(V_{0 n}=53 \mathrm{~m} / \mathrm{s}\right.$ and $\left.V_{0 t}=197.8 \mathrm{~m} / \mathrm{s}\right)$.

\subsubsection{Effect of film thickness}

To study the effect of film thickness on oblique impact, we simulate water films of $1 \mu \mathrm{m}$, $5 \mu \mathrm{m}$ and $20 \mu \mathrm{m}$. In all cases the drop has a diameter of $41.3-\mu \mathrm{m}$, impact angle of $45^{\circ}$ and 
normal impact velocity of $53 \mathrm{~m} / \mathrm{s}$. As shown in Figure 41, splashes are observed in all cases. On the downstream side, the vertical lamella is thin and breaks up almost immediately after its formation for the $1 \mu \mathrm{m}$ case, while the lamella is much thicker and lamella breakup is significantly delayed for the $20 \mu \mathrm{m}$ case. Because the formed lamella on the downstream side is mainly from liquid film, thinner film leads to thinner lamella, and thinner lamella is less stable and breakup happens sooner. On the upstream side, increasing film thickness also causes thicker lamella, and the upward motion of the thicker lamella is more prominent. Because thicker film in quiescent has more inertia and hence greater tendency to resist a change in motion, the lamella's upward motion becomes more prominent due to less resistance from air.

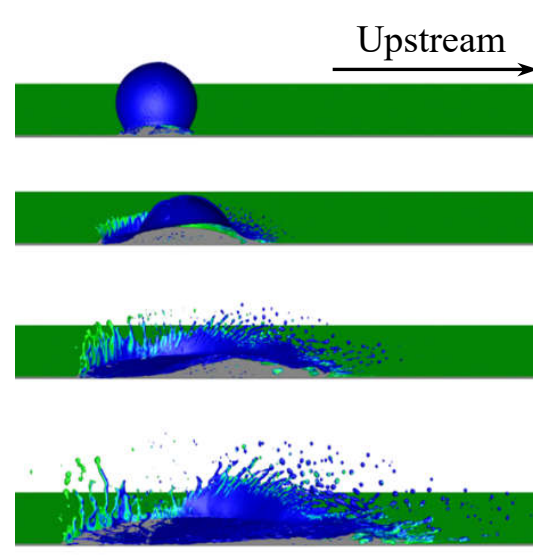

(a) $h_{0}=1 \mu \mathrm{m}, \delta=0.024$
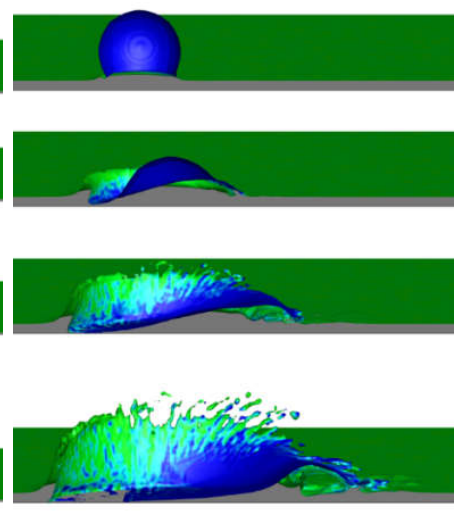

(b) $h_{0}=5 \mu \mathrm{m}, \delta=0.12$

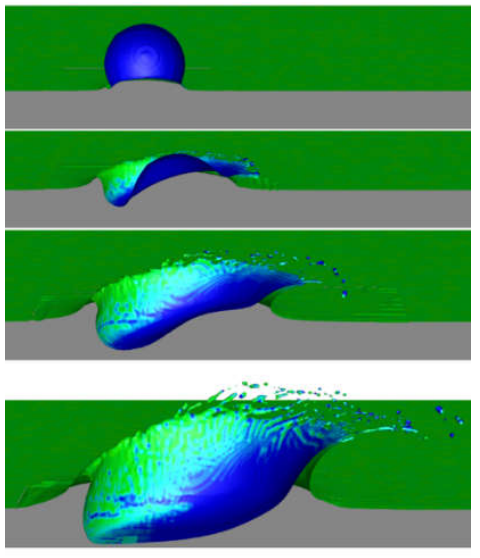

(c) $h_{0}=20 \mu \mathrm{m}, \delta=0.48$

Figure 41. Oblique impacts of 41.3- $\mu \mathrm{m}$ water drops on water films of $1 \mu \mathrm{m}, 5 \mu \mathrm{m}$ and 20 $\mu \mathrm{m}$. Impact angle is $45^{\circ}$ for all cases $\left(V_{0 n}=53 \mathrm{~m} / \mathrm{s}\right.$ and $\left.V_{0 t}=53 \mathrm{~m} / \mathrm{s}\right)$. 


\subsubsection{Effect of film-drop density ratio}

We vary the liquid film density while keeping the drop density constant to study the effect of density ratio of liquid film and drop. The simulations are performed in two-dimensional axisymmetric coordinate systems. Figure 42 shows numerical results of $41.3-\mu \mathrm{m}$-diameter water drops impact onto 5- $\mu \mathrm{m}$-thick liquid films of different densities. The impacts are normal and the impact velocity is $53 \mathrm{~m} / \mathrm{s}$ for all cases.

Figure 42 clearly shows that lowering the film density leads to early splashing: splashing occurs first at $0.07 \mu \mathrm{s}$ for the lowest film density case $\left(\rho_{\text {film }}=0.5 \rho_{\text {water }}\right)$ while splashing occurs last at $0.26 \mu$ s for the highest film density case $\left(\rho_{\text {film }}=1.5 \rho_{\text {water }}\right)$. For the $\rho_{\text {film }}=$ $0.5 \rho_{\text {water }}$ case, the crown formation and breakup are also observed at the early stage of the impact similar to the $\rho_{\text {film }}=\rho_{\text {water }}$ case. Then, from $t \approx 0.37 \mu \mathrm{s}$, the drop starts to spread beneath the film and a Kelvin-Helmholtz vortex is developed. Unlike the outward and upward motions of the expanding crown in the $\rho_{\text {film }}=\rho_{\text {water }}$ case, the crown collapses inward and splashing is suppressed at the later stage of the impact when $\rho_{\text {film }}=0.5 \rho_{\text {water }}$. For the $\rho_{\text {film }}=1.5 \rho_{\text {water }}$ case, splashing is also attenuated with lower crown height and smaller crown radius, because higher film density indicates that the crown has more inertia and less tendency to be pushed radially.

Figure 42 shows that at $t=0.07 \mu$ s in all cases a horn-shape jet is produced immediately after impact in the neck region where the drop contacts the liquid film. Although the jet shapes are similar and the crown radius $\frac{r_{c}}{D}=0.42$ for all cases obvious differences can be seen. For the $\rho_{\text {film }}=0.5 \rho_{\text {water }}$ case, the jet is from liquid film and already breaks up at 0.07 $\mu \mathrm{s}$; for the $\rho_{\text {film }}=\rho_{\text {water }}$ case, liquid from drop starts to move outward above the jet from 
liquid film; for the $\rho_{\text {film }}=1.5 \rho_{\text {water }}$ case, liquid from drop moves above and ahead of the jet from liquid film. At $t=0.18 \mu \mathrm{s}$, the crown tips of the $\rho_{\text {film }}=0.5 \rho_{\text {water }}$ case and the $\rho_{\text {film }}$ $=\rho_{\text {water }}$ case break up into secondary drops while the $\rho_{\text {film }}=1.5 \rho_{\text {water }}$ case shows the liquid from drop moves over the jet from liquid film and re-impacts the film to create a crater in front of the crown. At $t=0.26 \mu \mathrm{s}$, the remaining liquid film beneath the drop of the $\rho_{\text {film }}=$ $0.5 \rho_{\text {water }}$ case is much thinner compared to the other cases. 
(a)
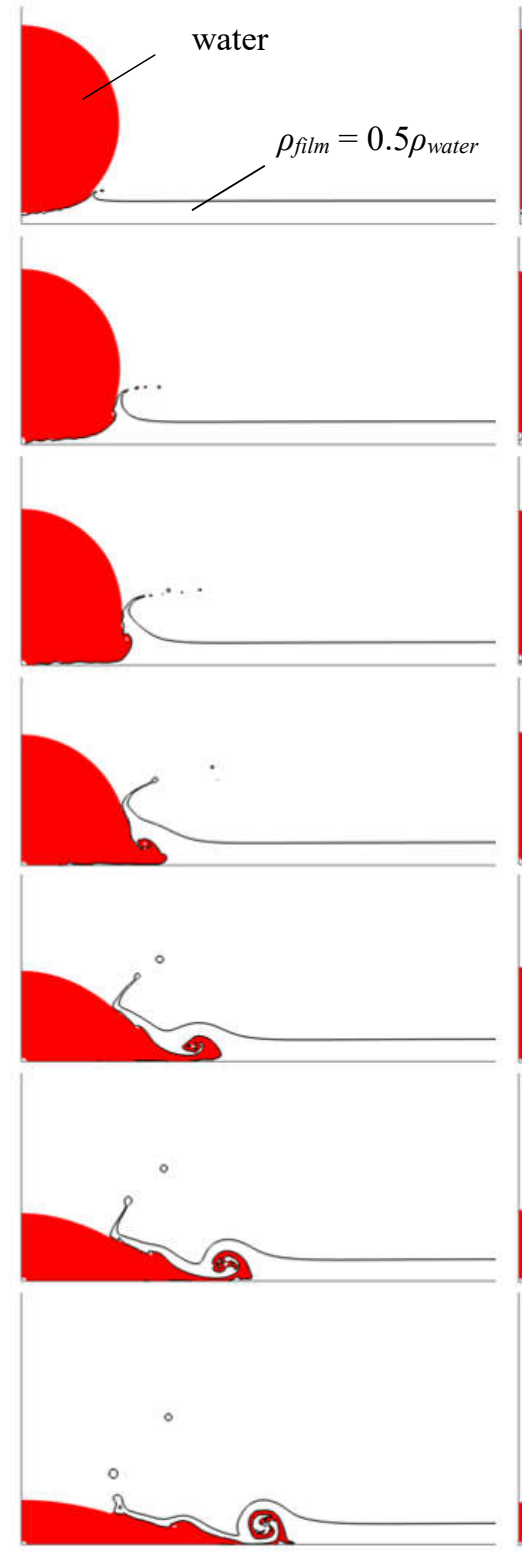

(b)

(c)

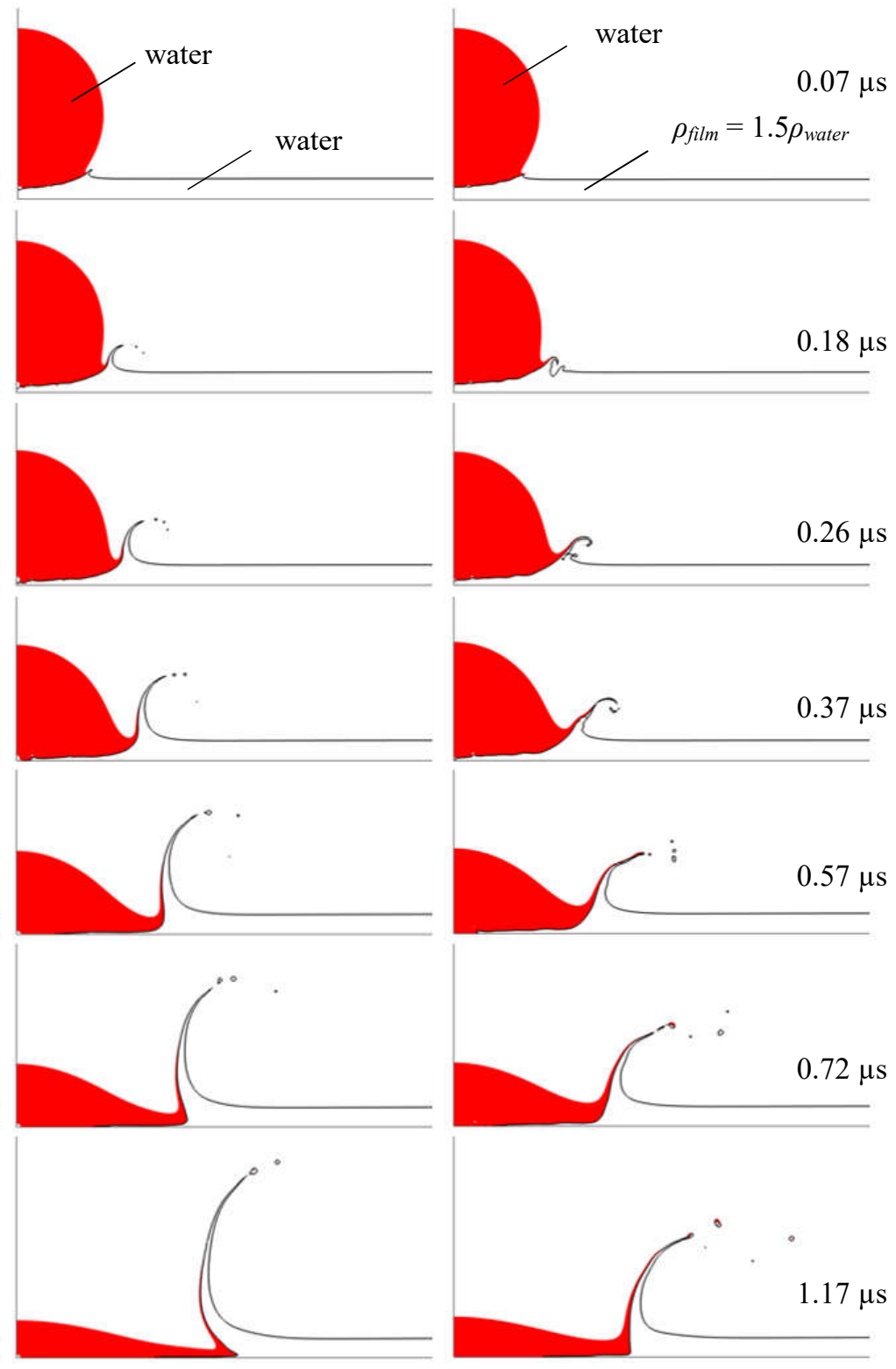

Figure 42. Normal impact of 41.3- $\mu \mathrm{m}$ water drop on 5 - $\mu \mathrm{m}$ liquid films with $53 \mathrm{~m} / \mathrm{s}$ impact velocity. (a): $\rho_{\text {film }}=0.5 \rho_{\text {water }} ;(\mathrm{b}): \rho_{\text {film }}=\rho_{\text {water }} ;(\mathrm{c}): \rho_{\text {film }}=1.5 \rho_{\text {water }}$. 


\subsection{Conclusions}

We numerically investigated the dynamics of high-speed oblique impact of a liquid drop on a thin liquid layer. The Navier-Stokes equations are solved using the projection method on Cartesian grids and the moment-of-fluid method is used to construct the interfaces. We validated the code first by comparing numerical results with experimental results and theoretical predictions and good agreement was achieved qualitatively and quantitatively. We studied the effect of impact angle by changing the tangential component of drop impact velocity while maintaining a constant normal component of impact velocity. Then we studied the effect of film thickness for oblique impacts with fixed impact angle. Finally, we studied the effect of film-drop density ratio by changing liquid film density.

Following important conclusions can be made from present study: (i) The tangential impact velocity of the incident drop affects the outcome of oblique drop impact. On the side behind the advancing drop, higher tangential velocity leads to lower lamella height and smaller lamella radius. On the side in front of the advancing drop, higher tangential velocity induced Kelvin-Helmholtz vortices development at the drop and liquid film interface and tends to suppress the evolution of lamella. (ii) Liquid film thickness affects the outcome of oblique drop impact. Thinner liquid film leads to thinner expanding crown and earlier crown breakup. (iii) Film density affects the outcome of oblique drop impact. Lower film density can prompt earlier splash but drop tends to move beneath the liquid film at the later stage of impact.

Our study revealed that tangential component of impact velocity is not negligible for oblique drop impingement, especially for high-speed impact. The tangential motion introduces large shear stress on the side in front of the advancing drop, which may prompt 
or suppress splash depending on the impact angle. The effect of film-drop density ratio is not observed in existing studies and certainly needs further investigation both experimentally and numerically. 


\section{CHAPTER 5. IMPACT OF MULTIPLE DROPS ON THIN LIQUID FILM}

\section{$5.1 \quad$ Introduction}

Understanding droplet impact on dry and wet surfaces is important for many industrial applications such as ink-jet printing and spray cooling. Extensive studies have been conducted to understand single droplet impact phenomenon (Worthington, 1908; Ching et al., 1984; Rein, 1993; Mundo et al., 1995; Yarin \& Weiss, 1995; Cossali et al., 1997; Samenfink et al., 1999; Weiss \& Yarin, 1999; Roisman \& Tropea, 2002; Thoroddsen, 2002; Josserand \& Zaleski, 2003; Rioboo et al., 2003; Cossali et al., 2004; Leneweit et al., 2005; Nikolopoulos et al., 2005; Xu et al., 2005; Yarin, 2006; Mukherjee \& Abraham, 2007; Nikolopoulos et al., 2007; Deegan et al., 2008; Okawa et al., 2008; Motzkus et al., 2009; Mani et al., 2010; Schroll et al., 2010; Latka et al., 2012; Mandre \& Brenner, 2012; Thoraval et al., 2012; Zhang et al., 2012; Tran et al., 2013; Shetabivash et al., 2014; Guo et al., 2016; Josserand \& Thoroddsen, 2016; Guo \& Lian, 2017). The drop impingement phenomenon could occur on dry or wet surfaces, however, a surface is commonly covered with a liquid layer formed by preceding drops. For drop impact on dry surfaces, Mundo et al. (Mundo et al., 1995) characterized the transition from deposition to splashing using a composite dimensional parameter $K\left(=R e^{0.25} \cdot W e^{0.5}\right)$ and the Reynolds number $(R e)$ and the Weber number $(\mathrm{We})$ are defined as

$$
R e=\frac{\rho D V_{0}}{\mu}, \quad W e=\frac{\rho D V_{0}^{2}}{\sigma},
$$


where $\rho, \mu$, and $\sigma$ are the liquid density, viscosity, and surface tension, respectively, and $D$ and $V_{0}$ are the drop diameter and impact velocity, respectively. When a drop impacts on a liquid thin film, bouncing, coalescence, and splashing may occur (Rein, 1993). Yarin suggested using a critical impact velocity as the splashing threshold for impact on wet surfaces (Yarin \& Weiss, 1995). Previous studies have identified a two-stage process in the drop splashing on a liquid thin film: in the first stage, a jet is formed from the neck region between the drop and the liquid layer and then this jet develops to form a crown shape; in the second stage, the crown tip breaks up to secondary drops due to instability. Cossali et al. (Cossali et al., 1997) and Yarin (Yarin, 2006) defined splashing as a phenomenon in which secondary droplets separating from the crown. Weiss and Yarin (Weiss \& Yarin, 1999) simulated drop impact on a liquid film to study the formation of jet in the neck region between drop and liquid film after impact and they found that the jet tip can detach or reconnect with liquid film. Thoroddsen (Thoroddsen, 2002) experimentally verified jet formation in the neck region between drop and liquid film. Randy et al. found that surface tension and viscosity act to inhibit splashing. In their experiment Cossali et al. (Cossali et al., 1997) noticed that the crown velocity and thickness is nearly independent of the Weber number. The crown is usually unstable and its tip can break up to form secondary droplets. Different mechanisms have been proposed to explain the tip breakup (Allen, 1975; Fullana \& Zaleski, 1999; Rieber \& Frohn, 1999; Roisman et al., 2006; Nikolopoulos et al., 2007; Krechetnikov \& Homsy, 2009).

Numerical investigations have been conducted to study normal impact of droplet on thin liquid films. Nikolopoulos et al (Nikolopoulos et al., 2005) used the volume of fluid method to study the formation of secondary droplets. Mukherjee and Abraham (Mukherjee \& 
Abraham, 2007) used lattice-Boltzmann model to study the influence of ambient gas density and viscosity and liquid film thickness. Shetabivash et al (Shetabivash et al., 2014) numerically investigated normal impingement of drop on a liquid layer and found that increasing gas density leads to reduction of crown radius and height evolution rate, while gas viscosity does not affect the rate of crown radius evolution.

Most previous work studies normal impact of a single drop. Few attempts have been made to investigate impact of multiple drops even though the influence of the drop interactions on the target surface can be significant (Tropea \& Roisman, 2000). Barnes et al demonstrated the interaction of crowns produced by the periodic impact of two drops on a spherical target (Barnes et al., 1999). Roisman et al. experimentally and theoretically studied the impact of multiple drops on a dry substrate (Roisman et al., 2002). They showed that the distance between the impacting drops has a significant effect on the behaviors of the liquid film formed on the substrate. These studies are for impact on dry substrate. Raman et al. (Raman et al., 2015) and Li et al. (Li et al., 2016) performed two-dimensional simulations to study the dynamic behavior of two droplets impinging normally on a liquid film using a Lattice Boltzmann method. They investigated the effects of distance between two impinging droplets, film thickness, viscosity, impinging velocity and density ratio on the crown structure. Xu et al. (Xu et al., 2014) numerically studied the normal impact of two droplets on a thin liquid film using the smoothed particles hydrodynamics (SPH) method. Most simulations are two-dimensional and are for normal impact. In this paper, we study oblique impact of multiple drops on a thin liquid film using a three-dimensional approach. 


\subsection{Results and discussion}

\subsubsection{Code validation: Normal impact on liquid film}

We first validate the code with experiments of normal impacts on liquid films. The experiments were conducted by Vander Wal et al. (Vander Wal et al., 2006). The liquid is $30 \%$ glycerin in water, the drop diameter is $2.0 \mathrm{~mm}$, the terminal impact velocity is 2.71 $\mathrm{m} / \mathrm{s}$, and the film thicknesses are $0.2 \mathrm{~mm}$ and $2 \mathrm{~mm}$. The resulting Weber number is 139 and Reynolds number is 1740 . Our simulations have the same initial conditions as the experiment setup. The results are shown in Figure 34 and the simulations are in good agreement with experiments. For impact on $0.2-\mathrm{mm}$ liquid film, a crown is formed and perturbation is observed at the crown rim. For impact on 2-mm liquid film, a short and thick crown is produced without perturbed liquid surface.

Experiment

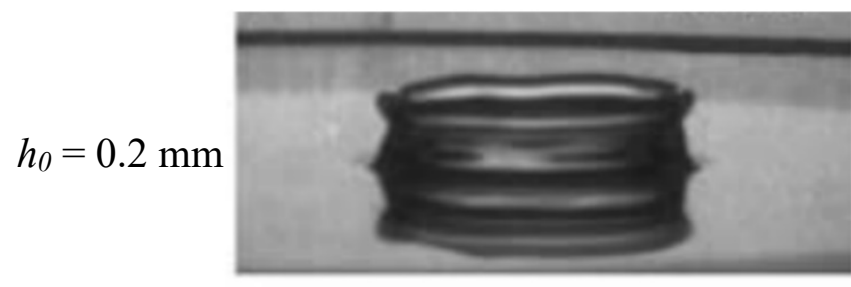

$h_{0}=2 \mathrm{~mm}$

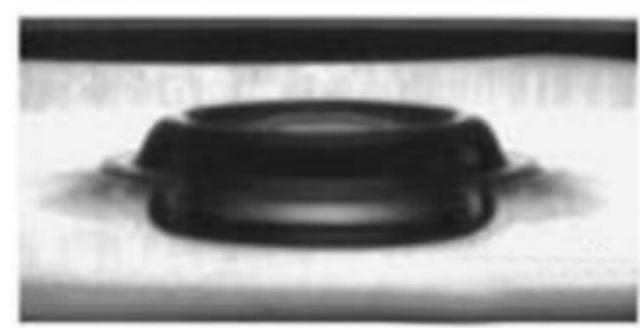

Simulation
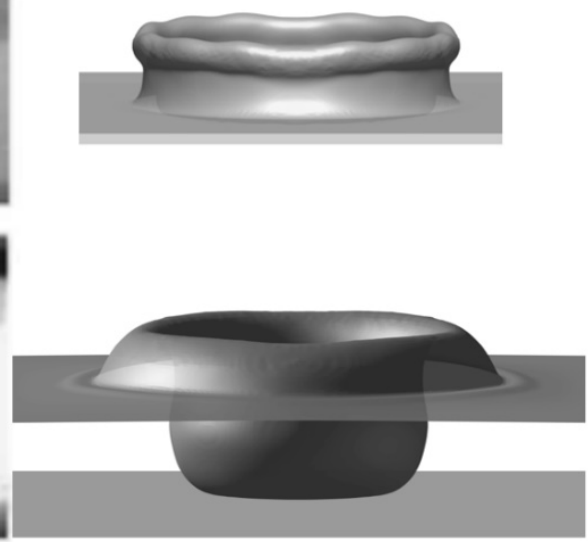

Figure 43. Normal impact of 2-mm-diameter glycerin-water droplet on same liquid film at $2.17 \mathrm{~m} / \mathrm{s}$. Top row: liquid film thickness is $0.2 \mathrm{~mm}$. Bottom row: liquid film thickness is 2 mm. Experiments were performed by Vander Wal et al. (Vander Wal et al., 2006). 


\subsubsection{Oblique impact of multiple drops on a thin film}

In most practical applications of droplet impingement, multiple droplets impact on surface rather than a single droplet as in spray cooling, inkjet printing, pesticide spraying, aircraft icing, etc. When a polydisperse spray impacts onto the surface covered by liquid layer created by previous drops, the expanding crowns interfere with each other. Also, normal impact is less common in practical applications as most drops impact onto surfaces with different impact angles. To investigate the effect of oblique impact on droplet interaction behavior, we simulate oblique impact of multiple drops on a thin liquid film.

A schematic of the simulation setup of two neighboring water droplets impacting onto thin liquid film is shown in Figure 44. The liquid drop diameter is $D_{0}$ and the liquid film thickness is $h_{0}$. The drop is surrounded by air above the liquid film. The impact angle is $\theta$ and the impact velocity is $V_{0}$, and $V_{0 t}$ and $V_{0 n}$ are the tangential and normal components of impact velocity respectively. The dimensionless time is defined as $T=\frac{V_{0 n} \cdot t}{D_{0}}$, and the dimensionless film thickness is defined as $\delta=\frac{h_{0}}{D_{0}}$. Due to symmetry of the problem, the computational domain only contains half of the droplets for oblique impact. The tangential velocity component points to right in Figure 44 and we define the right side of the drop as upstream side and left side as downstream side. 


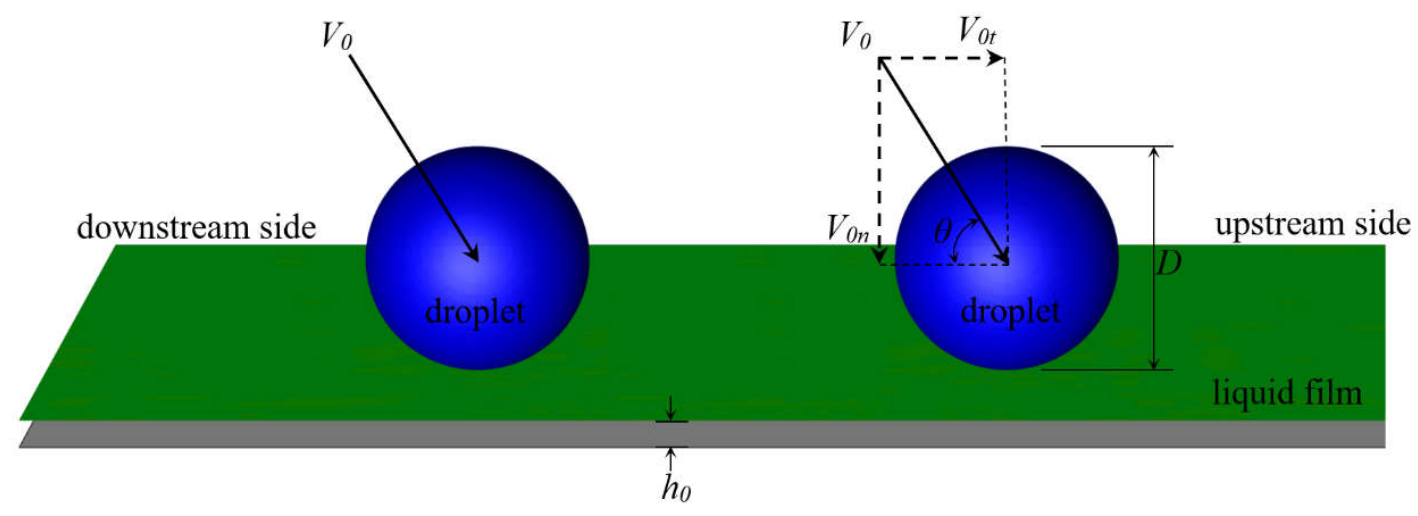

Figure 44. Simulation setup of two neighboring droplets oblique impact on a liquid film.

Simulations of two neighboring water droplets simultaneously impact on a 5 - $\mu$ m-thick water film are performed. The diameters of the two drops are both $41.3 \mu \mathrm{m}$. The normal impact velocities, $V_{0 n}$, are $3.5 \mathrm{~m} / \mathrm{s}$ and $53 \mathrm{~m} / \mathrm{s}$ and the impact angles are both $45^{\circ}$. Figure 45 and Figure 47 show the numerical results of the low-speed impact case and the highspeed impact case, respectively. When the impact velocity is lower than threshold, no splashing is observed, as shown in Figure 45 and Figure 46. Short and thick crowns are formed after the impacts, propagating in both the upstream and downstream directions. Due to the tangential impact velocity, the crown moves faster and further in the upstream direction and the crown is also taller and thicker on the upstream side. At the region between the two drops, the crown is even taller, possibility from superposition of crowns formed by two drops. After $t=20.9 \mu \mathrm{s}$, as shown in Figure 45 and Figure 46, the solid surface starts to dry out at the impact sites and the emerged solid areas become larger with the propagation of crowns. 


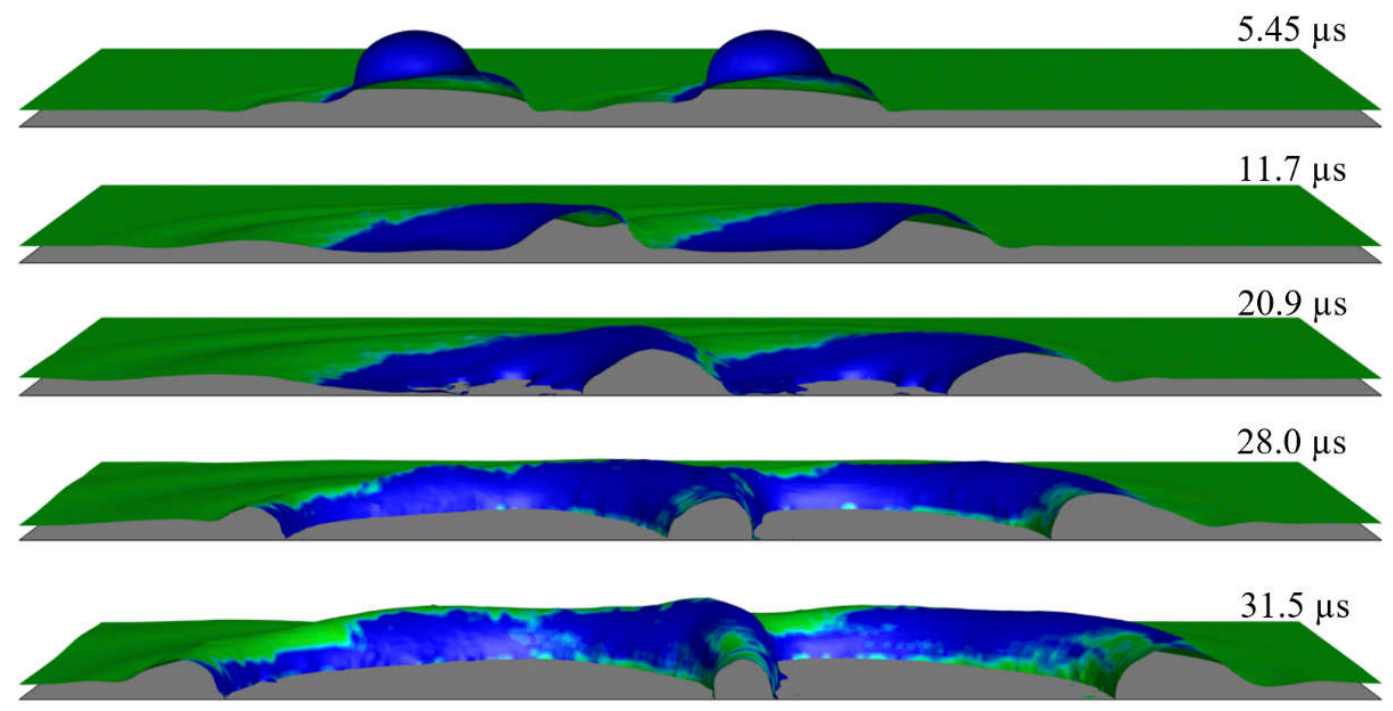

Figure 45. Oblique impact of two neighboring water droplets impact on a 5- $\mu \mathrm{m}$ water film simultaneously. Impact angle is $45^{\circ}$ and normal impact velocity component is $3.5 \mathrm{~m} / \mathrm{s}$.
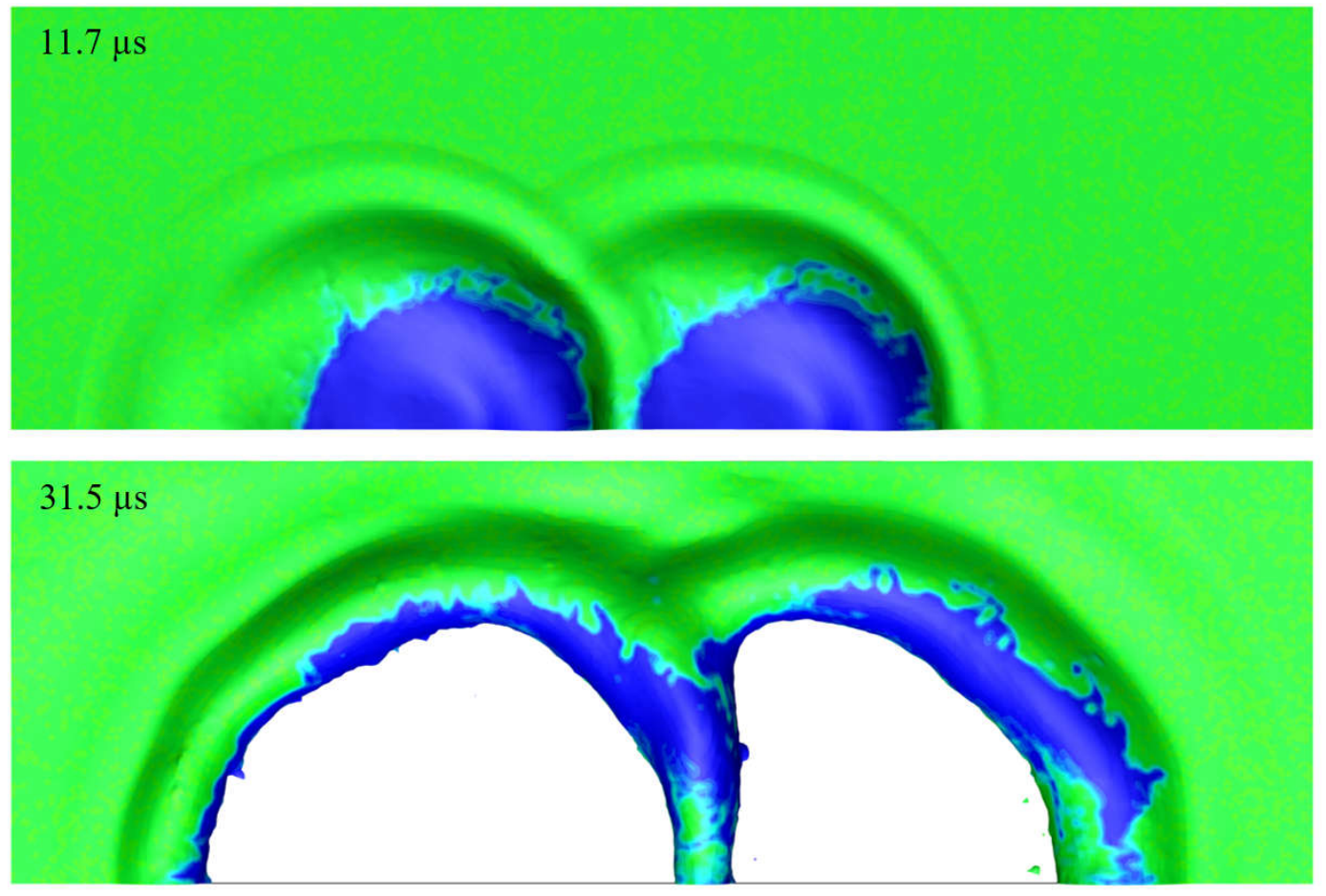

Figure 46. Top views of the two neighboring water droplets impact on water film in Figure 45. 
Figure 47 and Figure 48 show the impact at much higher impact velocity, which resulting crown splashing. A crown is formed after the impact, however, the lamella shapes on the upstream and downstream sides are different. On the upstream side, the lamella is in a thin horizontal horn shape. On the downstream side, the lamella is in a thick vertical wall shape. Also, the lamella moves faster on the upstream side due to the tangential impact velocity. Before the lamellae interact, similar crown shapes can be observed for both drops. Then the fluid behavior becomes complicated after the lamellae originated from two drops interact with each other. Because of the tangential motion of the drops, the interaction region between the two drops mainly moves in the upstream direction. At $t=4.24 \mu \mathrm{s}$ as in Figure 47 and Figure 48, the lamella breaks up into liquid fingers and secondary droplets, which are higher in the interaction region than the upstream and downstream sides. The reason for the lamella breaks up higher in the interaction region is that the lamellae move toward each other which excites the motion upward. 
$0.39 \mu \mathrm{s}$
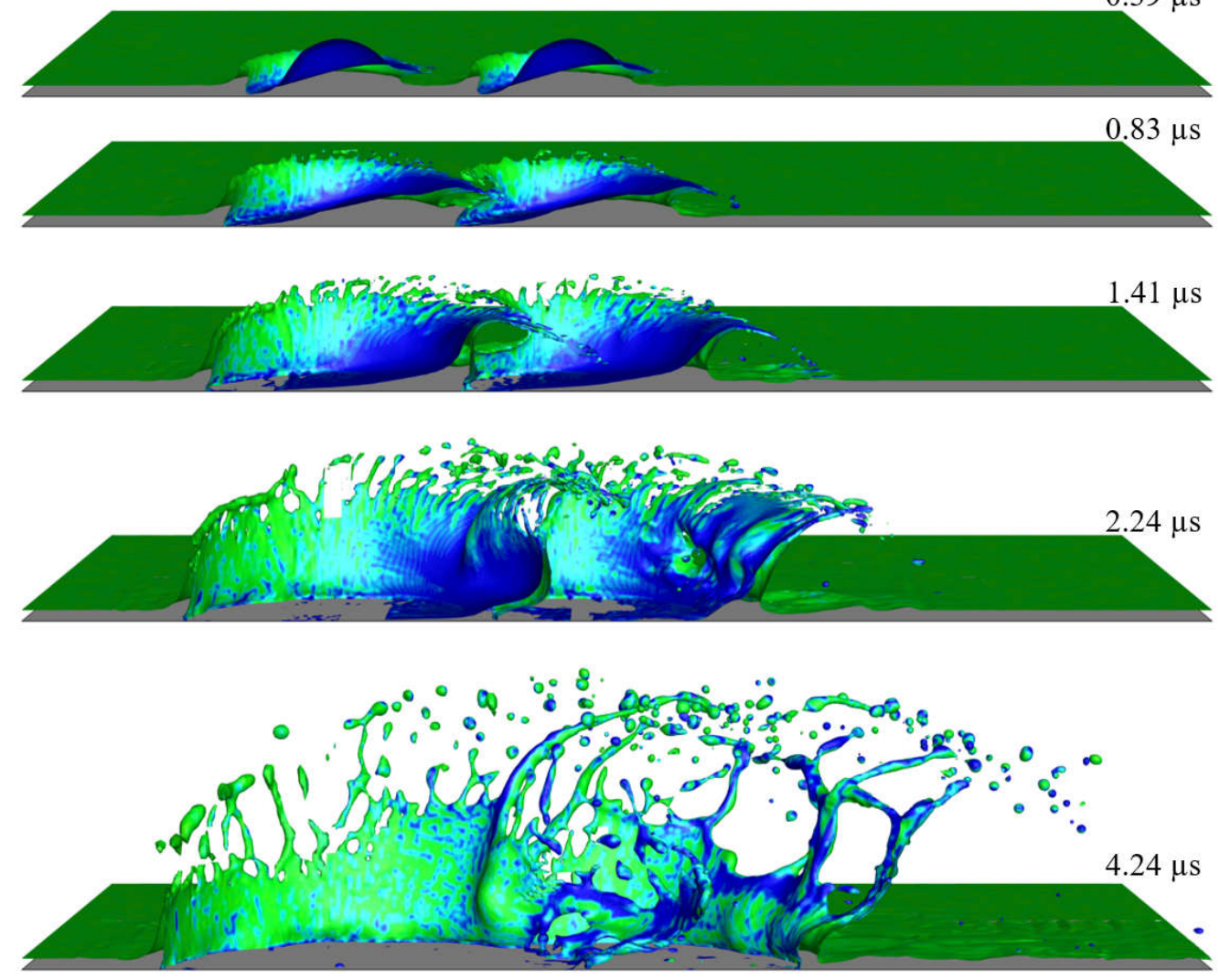

Figure 47. Oblique impact of two neighboring water droplets impact on a 5- $\mu \mathrm{m}$ water film simultaneously. Impact angle is $45^{\circ}$ and normal impact velocity component is $53 \mathrm{~m} / \mathrm{s}$. 

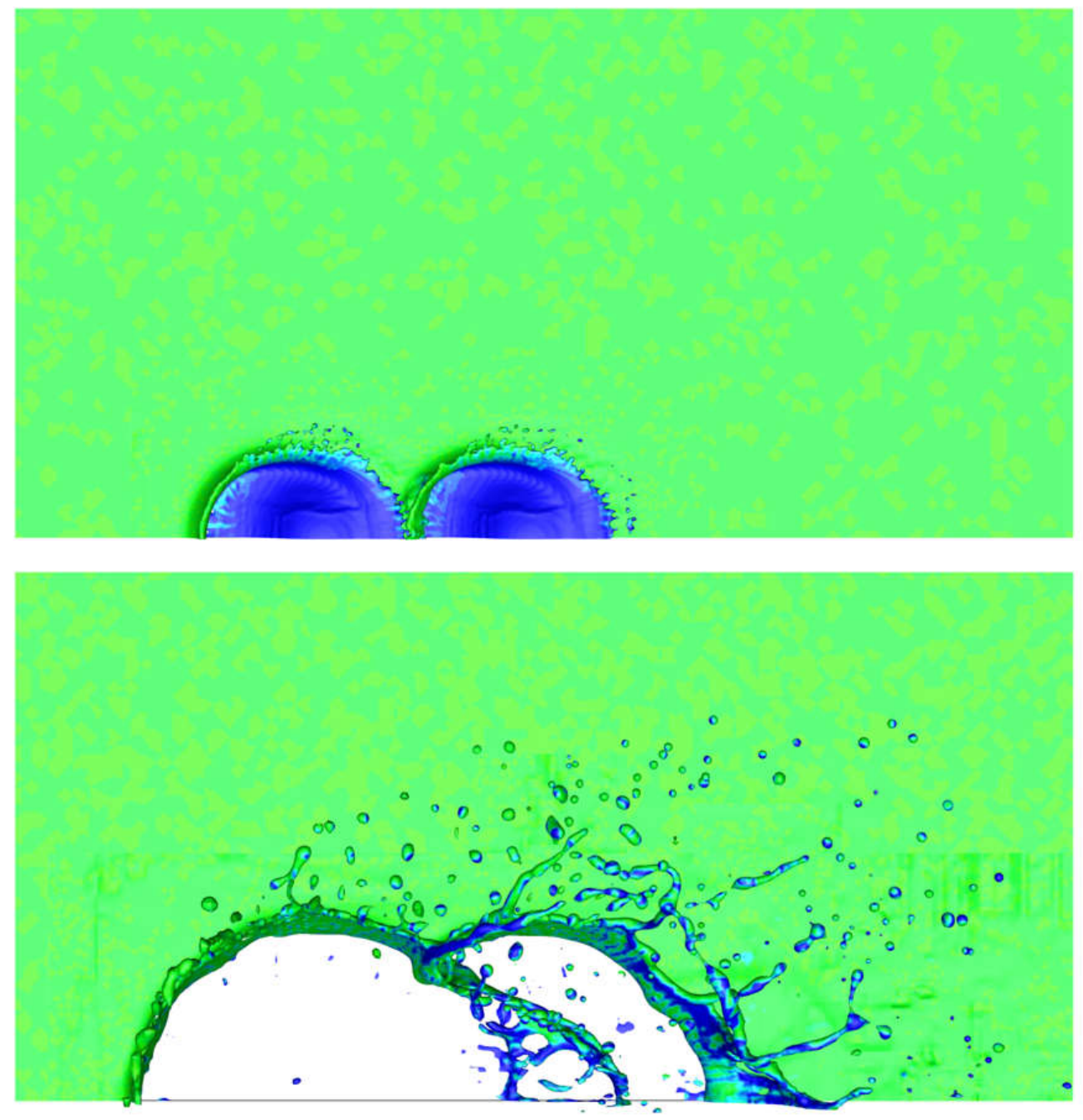

Figure 48. Top views of the two neighboring water droplets impact on water film in Figure 47.

Two water drops impact on thin film at different time instants is also simulated. The results are shown in Figure 49 and Figure 50. The right drop impacts first and the left drop impacts on the liquid film after $1 \mu \mathrm{s}$. The interaction behavior is similar to the simultaneous impact case in Figure 47. At $t=1.686 \mu \mathrm{s}$, the lamella formed on the upstream side of the left drop strikes the vertical lamella formed on the downstream side of the right drop. After the 
lamellae interaction, the upstream lamella cuts through the downstream lamella and they move upstream jointly as shown in Figure 50. Solid surface dry out is also observed, and because no liquid film still exists on the solid surface inside the crater, the lamella formed between the two drops moves faster.

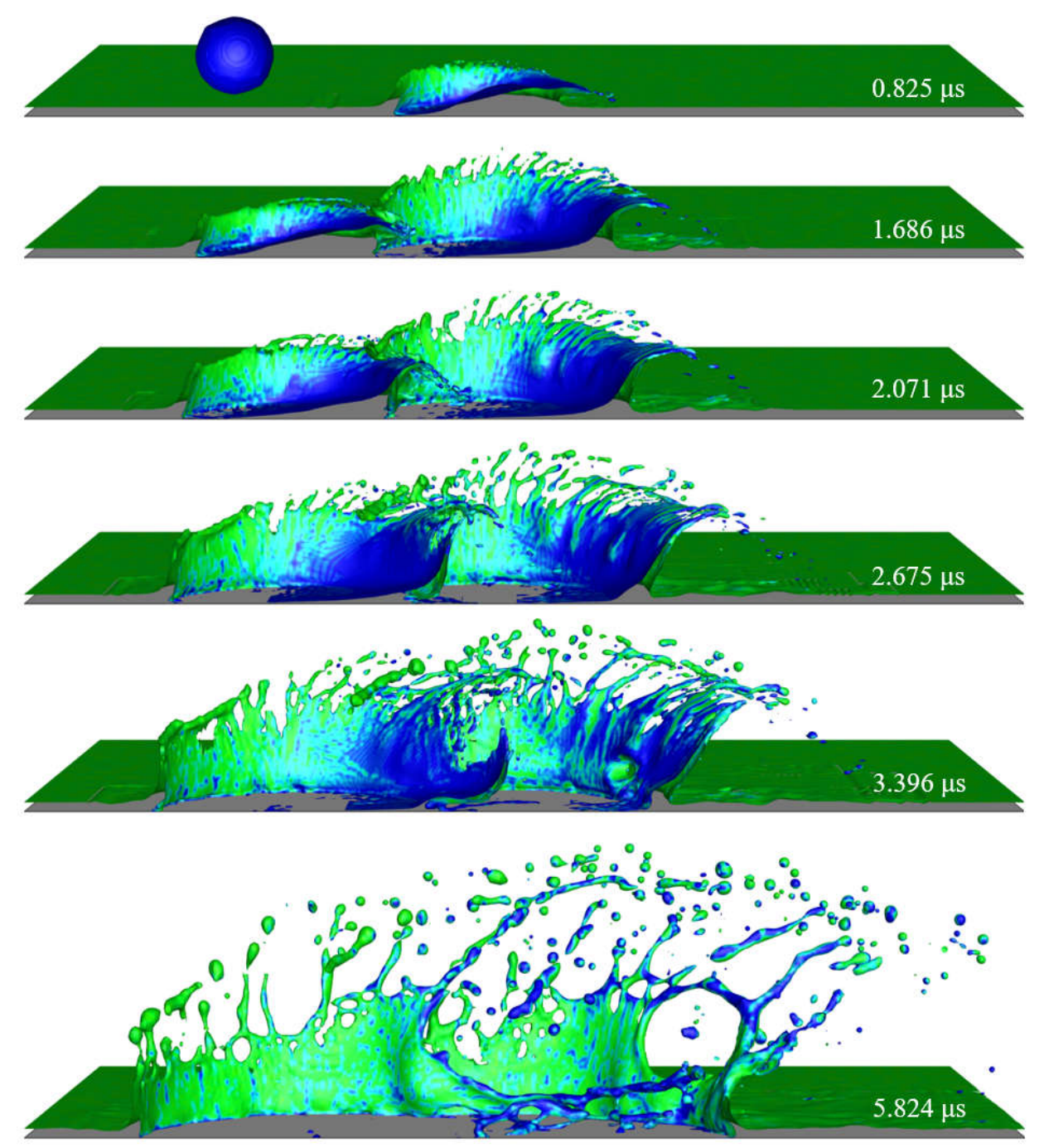

Figure 49. Oblique impact of two neighboring water droplets impact on a 5- $\mu \mathrm{m}$ water film asynchronously. Impact angle is $45^{\circ}$ and normal impact velocity component is $53 \mathrm{~m} / \mathrm{s}$. 

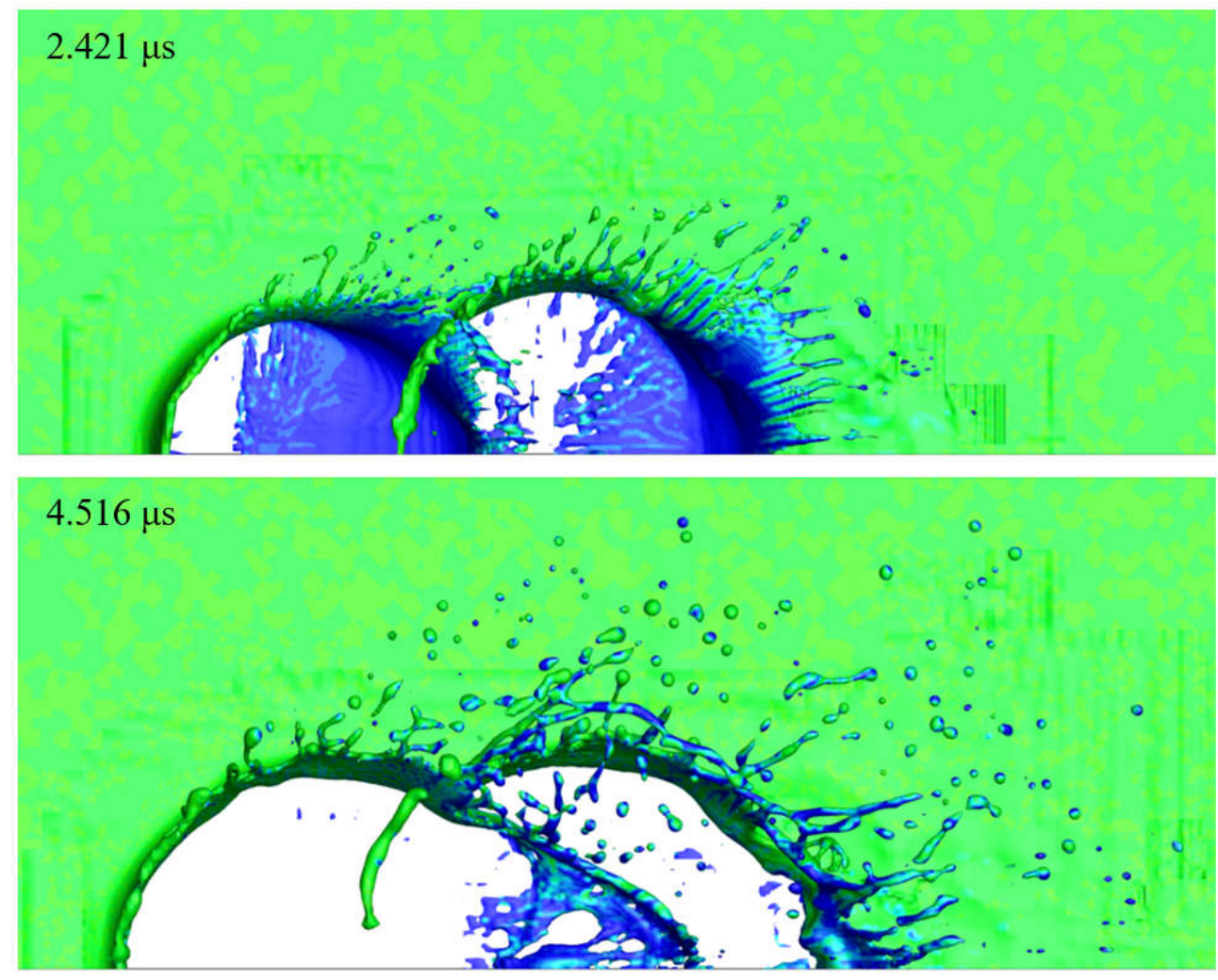

Figure 50. Top views of the two neighboring water droplets impact on water film in Figure 49.

\subsection{Conclusions}

We numerically investigated the dynamics of oblique impact of multiple drops on a liquid film. The Navier-Stokes equations are solved using the projection method on Cartesian grids and the moment-of-fluid method is used to construct the interfaces. We validated the code by comparing our numerical results to experimental results and they showed good agreement. Then we simulated two neighboring water droplets impacting on a water film at $45^{\circ}$ impact angle. In all our simulations, the dimensionless film thickness is 0.024 and dry out of the solid surface is observed at the impact site. When the two drops impact on 
thin film simultaneously, deposition occurs for low-speed impact and splashing occurs for high-speed impact. When the drop impact inertia is low, short and thick crowns are formed after the impact, and the crown is taller at the interaction region between the two neighboring drops. When the drop impact inertia is high, crowns are formed and break up into liquid fingers and secondary droplets, and strong interaction between the lamellae formed by the two neighboring drops is observed. The simulation of two drops high-speed impact asynchronously shows similar phenomenon to high-speed simultaneous impact. 


\section{CHAPTER 6. CALCULATION OF WATER COLLECTION EFFICIENCY}

\subsection{Introduction}

\subsubsection{Water collection efficiency and its importance}

Aircraft icing under extreme weather conditions is one of the major serious hazards to affect aviation safety. Ice accretion on the external parts of an aircraft during flight is caused by freezing rain/drizzle or supercooled water droplet. And icing on aircraft could alter the effective wing geometry and impact the performance and control of the aircraft (Bragg et al., 1986; Ranaudo et al., 1991). Therefore, performing aircraft icing analysis is crucial for assessing the effect of ice accretion on an aircraft. One important stage in aircraft icing analysis is to determine water collection efficiency and impact limit.

The local water collection efficiency, $\beta$, is defined as the volume flux ratio of an infinitesimal area with water droplets, $d A_{\infty}$, to the corresponding impingement area on the airfoil, $d A$, by using the droplet trajectory data (Tan, 2004). As shown in Figure 51, the local water collection efficiency, $\beta$, can be expressed as:

$$
\beta=\frac{d A_{\infty}}{d A}
$$

Because water droplets can splash after impact on the surface, the local water collection efficiency is modified with the consideration of droplet splashing phenomenon and is expressed as: 


$$
\beta_{\text {splash }}=\frac{d A_{\infty}}{d A}\left(\frac{\dot{m}_{\text {film }}}{\dot{m}_{\text {film }}+\dot{m}_{\text {splash }}}\right)
$$

where $\dot{m}_{f i m}$ denotes the mass depositing onto the surface and $\dot{m}_{\text {splash }}$ denotes the mass loss due to splashing. The impact limit is defined as the upper and lower limits that water droplets can impinge on the surface. Deicing devices are required within the impact limit to remove ice. Experiments have shown that airspeed, droplet size, airfoil type and body size are primary parameters for determining water collection efficiency and impact limit (Gelder et al., 1956; Lewis \& Ruggeri, 1957; Papadakis et al., 1989).

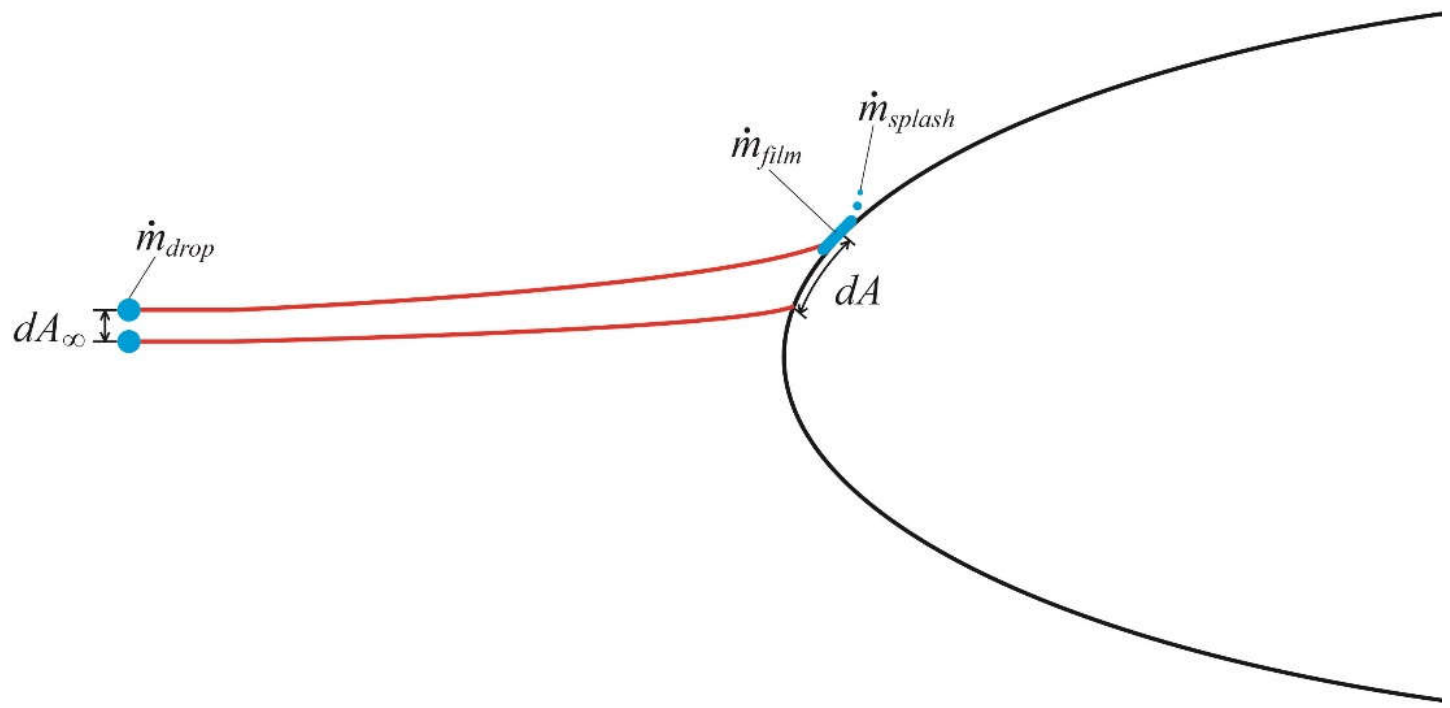

Figure 51. Definition of water collection efficiency (Tan, 2004).

\subsubsection{Existing methods to predict water collection efficiency}

Various approaches have been proposed to obtain the local water collection efficiency. Experimental studies started in the early 1950's and dyed water droplets were sprayed onto the surface of the body covered with absorbent blotting paper (Papadakis et al., 1989; 
Papadakis et al., 1994; Papadakis et al., 2002). Local collection efficiency is obtained using a laser reflectance spectroscopy method by analyzing the amount of dye on the blotter paper in a given time interval.

Numerical studies are also conducted to investigate local collection efficiency and ice accretion and different models have been proposed and developed. Most of these models sequentially solve different sets of equations to obtain airflow, droplet trajectory and impingement, heat transfer and phase change to study aircraft ice accretion. Among them, FENSAP-ICE, initially proposed by Bourgault et al. (Bourgault et al., 1999; Morency et al., 2001), uses an Eulerian approach to account for water droplets impingement. FENSAPICE solves the liquid phase independent of the gas phase and therefore the water droplets do not affect the airflow. This method is validated by successfully predicting local collection efficiency of small water droplets. LEWICE (Potapczuk et al., 1993; Wright, 2005; Wright, 2006) (LEWis ICE accretion program) is the main icing code used in the U.S. and was developed by NASA Glenn Research Center. LEWICE uses the panel method to compute the trajectory of each droplet based on a force balance. The droplet is assumed to be a rigid sphere and does not affect the airflow. Drop breakup and splashing are considered in LEWICE for calculating local collection efficiency and good agreement is achieved with experimental data. ONICE2D (ONERA 2D icing suite) (Villedieu et al., 2014) uses a Lagrangian approach to calculate the deposition probability of each droplet, and then determines whether the droplet fully deposits on the solid surface or collides with secondary droplets from previous droplet impacts. ONICE2D also considers the effect of droplet shape on drag force when calculating trajectory. Table 4 lists some existing models for calculating water collection efficiency and their approaches. Most of the models 
consider the effect of air flow on the droplet (the drag and the trajectory), but the effect of droplet on the flow field is largely ignored. Droplet splashing has a significant effect on the water collection efficiency. However, most studies either ignore droplet splashing or are based on simplified empirical splashing models (Bilanin, 1991).

Table 4. Selected existing models for calculating water collection efficiency

\begin{tabular}{|c|c|c|c|c|c|c|}
\hline & $\begin{array}{l}\text { Lagrangian } \\
\text { or Eulerian }\end{array}$ & Airflow & $\begin{array}{l}\text { Interaction } \\
\text { between } \\
\text { droplets and } \\
\text { airflow }\end{array}$ & $\begin{array}{l}\text { Droplet } \\
\text { deformation }\end{array}$ & Drag model & $\begin{array}{l}\text { Droplet } \\
\text { splashing }\end{array}$ \\
\hline $\begin{array}{l}\text { LEWICE } \\
\text { (Potapczuk et } \\
\text { al., 1993; } \\
\text { Wright, } \\
\text { 2005; } \\
\text { Wright, } \\
\text { 2006) }\end{array}$ & Lagrangian & $\begin{array}{l}\text { Panel } \\
\text { method }\end{array}$ & $\begin{array}{l}\text { Droplets do not } \\
\text { affect airflow }\end{array}$ & No & Drag for spheres & Yes \\
\hline $\begin{array}{l}\text { FENSAP- } \\
\text { ICE } \\
\text { (Bourgault et } \\
\text { al., 1999; } \\
\text { Morency et } \\
\text { al., 2001) }\end{array}$ & Eulerian & $\begin{array}{l}\text { Euler or } \\
\text { Navier- } \\
\text { Stokes } \\
\text { solver }\end{array}$ & $\begin{array}{l}\text { Droplets do not } \\
\text { affect airflow }\end{array}$ & No & $\begin{array}{l}\text { Shiller Naumann } \\
\text { drag model }\end{array}$ & No \\
\hline $\begin{array}{l}\text { ONICE2D } \\
\text { (Villedieu et } \\
\text { al., 2014) }\end{array}$ & Lagrangian & $\begin{array}{l}\text { Panel } \\
\text { method }\end{array}$ & $\begin{array}{l}\text { Droplets do not } \\
\text { affect airflow }\end{array}$ & Yes & $\begin{array}{l}\text { Shiller Naumann } \\
\text { drag model }\end{array}$ & Yes \\
\hline $\begin{array}{l}\text { CANICE } \\
\text { (Morency et } \\
\text { al., 1999) }\end{array}$ & Lagrangian & $\begin{array}{l}\text { Panel } \\
\text { method }\end{array}$ & $\begin{array}{l}\text { Droplets do not } \\
\text { affect airflow }\end{array}$ & No & - & No \\
\hline $\begin{array}{l}\text { TURBICE } \\
\text { (Makkonen } \\
\text { et al., 2001) }\end{array}$ & Lagrangian & $\begin{array}{l}\text { Panel } \\
\text { method }\end{array}$ & $\begin{array}{l}\text { Droplets do not } \\
\text { affect airflow }\end{array}$ & No & - & No \\
\hline $\begin{array}{l}\text { Da Silveria } \\
\text { et al. (Da } \\
\text { Silveira et } \\
\text { al., 2003) }\end{array}$ & Eulerian & $\begin{array}{l}\text { Navier- } \\
\text { Stokes } \\
\text { solver }\end{array}$ & $\begin{array}{l}\text { Mutual } \\
\text { interaction }\end{array}$ & No & $\begin{array}{l}\text { Drag coefficients for } \\
\text { spherical and non- } \\
\text { spherical particles }\end{array}$ & No \\
\hline
\end{tabular}




\subsubsection{Effect of large droplets on water collection efficiency}

Droplet splashing is mainly caused by supercooled large droplet (SLD). SLD usually refers to liquid droplet with diameter larger than $50 \mu \mathrm{m}$. SLDs remain in liquid form even when the temperature is below freezing point because the lack of freezing nuclei. Aircraft icing under SLD condition imposes great danger to aviation safety and has been recognized as a significant aviation hazard (Potapczuk et al., 1993; Bragg, 1996). Several fatal aviation accidents have been caused by SLDs and recent studies indicate icing due to SLDs occurs more frequently than initially realized (Bragg, 1996; Petty \& Floyd, 2004). Icing due to SLD condition has been extensively studied in the recent years and has been included in the regulations of Federal Aviation Administration (FAA) (Administration, 2014) and European Aviation Safety Agensy (EASA) (Agency, 2015).

Due to its large size, SLD can splash into secondary droplets when it impacts the surface of the body. Consequently, droplet splashing reduces the amount of water depositing onto the and accordingly affects the local collection efficiency significantly. Besides, the splashed droplets can re-impinge onto unprotected surfaces to form ice (Bragg, 1996). Therefore, accurately predicting the splashing physics and mass loss is crucial in calculating local collection efficiency. However, the underlying mechanism of large droplet splashing is still not fully understood (Rutkowski et al., 2003; Wright \& Potapczuk, 2004; Wright, 2006). 


\subsection{Procedure of calculating water collection efficiency}

The calculation of water collection efficiency is through a two-step process. In the first step, the efficiency is calculated based on the droplet trajectory without considering the droplet splashing. In the second step, the efficiency is adjusted based on the output of splashing. The procedure is shown in Figure 52. In the first step, we calculate the droplet trajectories based on the size group and use a 7-bin droplet distribution to represent the median volume diameter (MVD). In our simulation, we position the group of droplets of same size in front of the sphere and assign them the same velocity as the surrounding air. Simulation is then first conducted to obtain the trajectory of each droplet. The water collection efficiency based on the area ratio is calculated. For any droplet which eventually hits the solid surface, its terminal speed and impact angle right before hitting the airfoil are then recorded. A separated simulation with fine mesh is used to calculate the volume of the splashed droplets of these droplets based on the recorded terminal speed and impact angle. The water collection efficiency is then adjusted based on the splashed volume. These steps repeat until all groups are evaluated. 


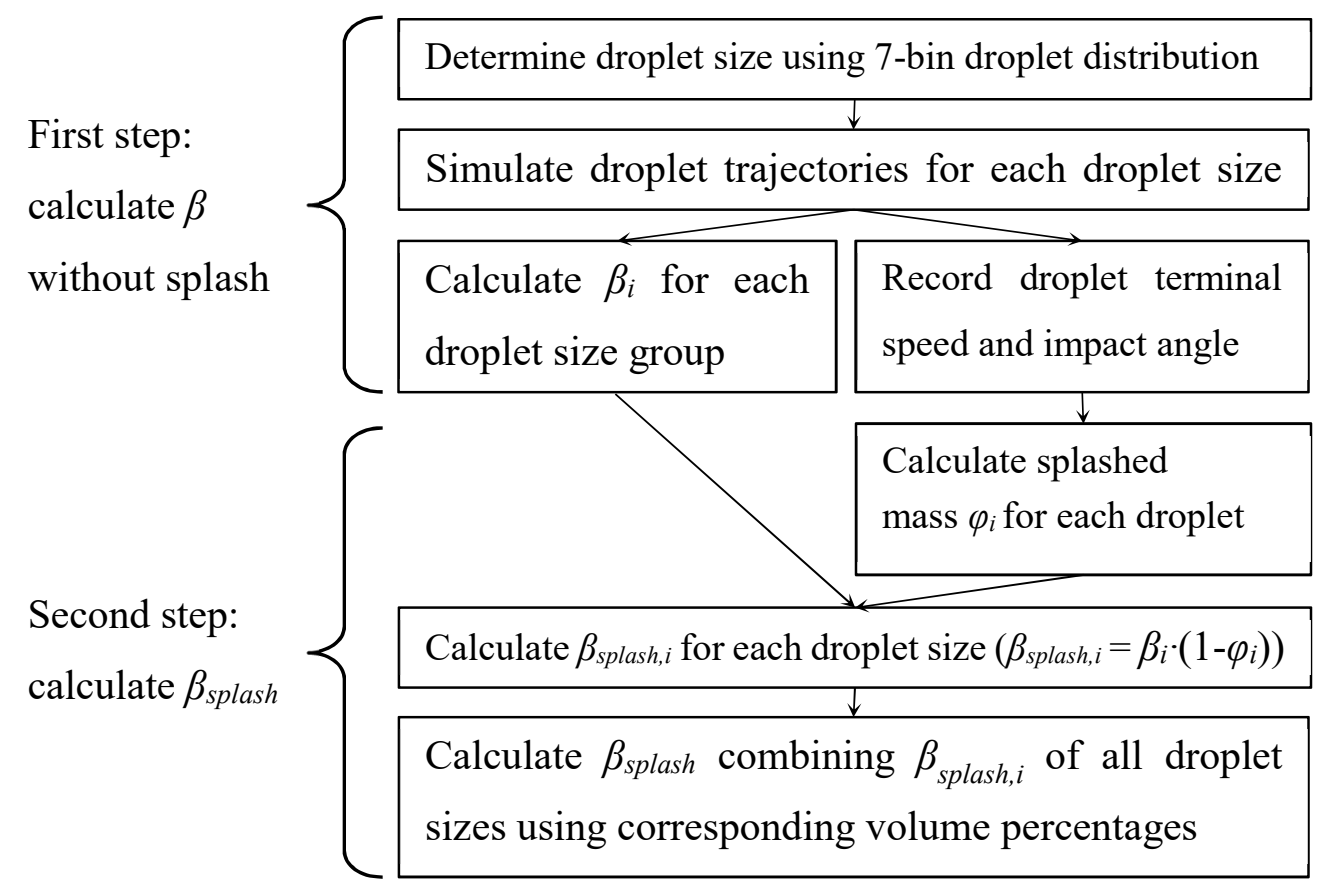

Figure 52. Procedure of calculating water collection efficiency

\subsection{Results and Discussion}

We first validate our code by comparing with experimental results. Then we study highspeed droplet impact on a dry surface and on a wet surface at the conditions pertinent to aircraft icing. Last we study water collection efficiency of a sphere and an MS(1)-317 airfoil. In all simulations, we assume the water is at room temperature and a static contact angle of $70^{\circ}$. A dynamic contact angle model of Jiang et al. (Jiang et al., 1978) is used to model dynamic change of contact angle with contact line velocity.

\subsubsection{Code validation: droplet impact on dry surface}

Mundo et al. (Mundo et al., 1995) experimentally studied droplet impingement onto a rotating disc and performed a statistical analysis of the splashed secondary droplets. The normal impact velocity of droplet and tangential velocity of rotating disc lead to oblique 
impact. They concluded that the normal velocity component determines the size distribution of the secondary droplets. To validate our code, we select one case from their study to simulate. The size and velocity distributions of the splashed droplets are then analyzed and compared with their experimental results. Since those splashed droplets have irregular shapes, we use equivalent droplet diameter which makes a sphere to have the same volume as an irregular droplet. The velocity of each splashed secondary droplet is the mass averaged velocity. The shooting angle is the angle between the bouncing velocity vector and the tangent direction of the solid surface at the location of bouncing.

The experiment used a solid dry surface. The initial diameter of the droplet, $D_{0}$, is $100 \mu \mathrm{m}$, the impingement velocity is $15 \mathrm{~m} / \mathrm{s}$, the impingement angle, $\alpha$, is $37^{\circ}$, leading to a normal impact velocity, $V_{0}$, of $12 \mathrm{~m} / \mathrm{s}$. The liquid viscosity is $2.76 \mathrm{mPa} \cdot \mathrm{s}$, and the surface tension is $22 \mathrm{mN} / \mathrm{m}$. The resulting $R e$ is 1182 and $W e$ is 868 . Note that both $R e$ and $W e$ are calculated using the normal impact velocity. The resulting $K$ value is 186.6 , which is higher than the splashing threshold of 57.7 suggested by Mundo et al. (Mundo et al., 1995). Grid sensitivity analysis was performed. With 2 levels of mesh refinement, the grid with an effective grid resolution of 116 cells per initial droplet diameter leads to a converged solution. Figure 53 shows the droplet impact and the subsequent splashing phenomena. Right after contact with the solid surface, a thin liquid sheet is formed on the solid surface and moves radially outward. The sheet soon breaks up into small secondary droplets. As the principle sheet breaks up at its perimeter into relatively larger droplets. Splashing is more prominent at the droplet leading edge than at the trailing edge: less number of secondary droplets was observed at the trailing edge and their sizes are considerably smaller than those at the leading edge. The size distribution of the secondary droplets is 
shown in Figure 54. The secondary droplet diameter is normalized using the diameter of the primary droplet. Our simulation shows qualitative agreement with the experiment. The numerical results are measured $59.1 \mu \mathrm{s}$ after impact but the time instant of the experiment results is not reported by Mundo et al. (Mundo et al., 1995), which may contribute to the discrepancy.

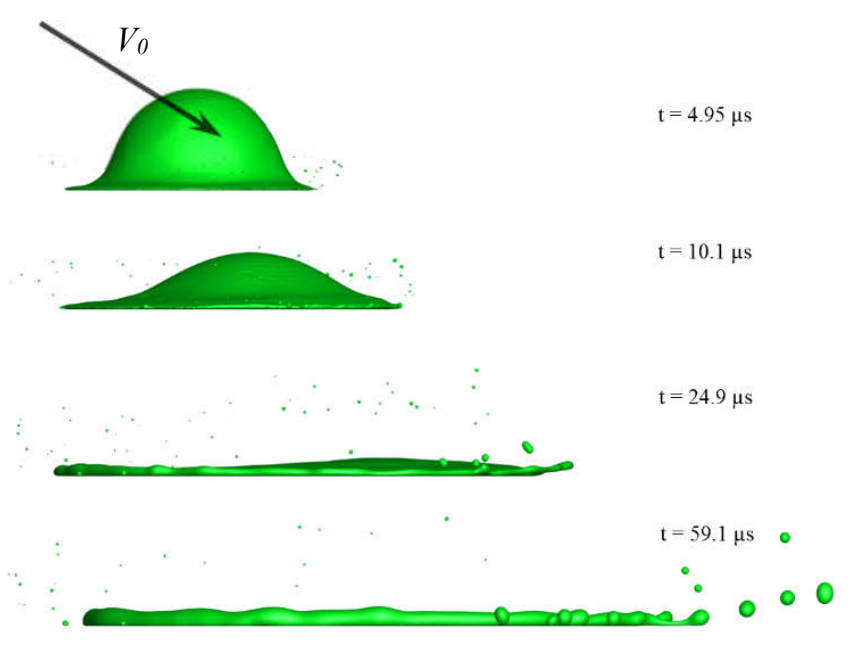

Figure 53. Simulation of oblique impact on dry solid surface, $D_{0}=100 \mu \mathrm{m}, V_{0}=12 \mathrm{~m} / \mathrm{s}, \alpha$ $=37^{\circ}$, and $K=186.6$. Secondary droplets are larger on the upstream side. 


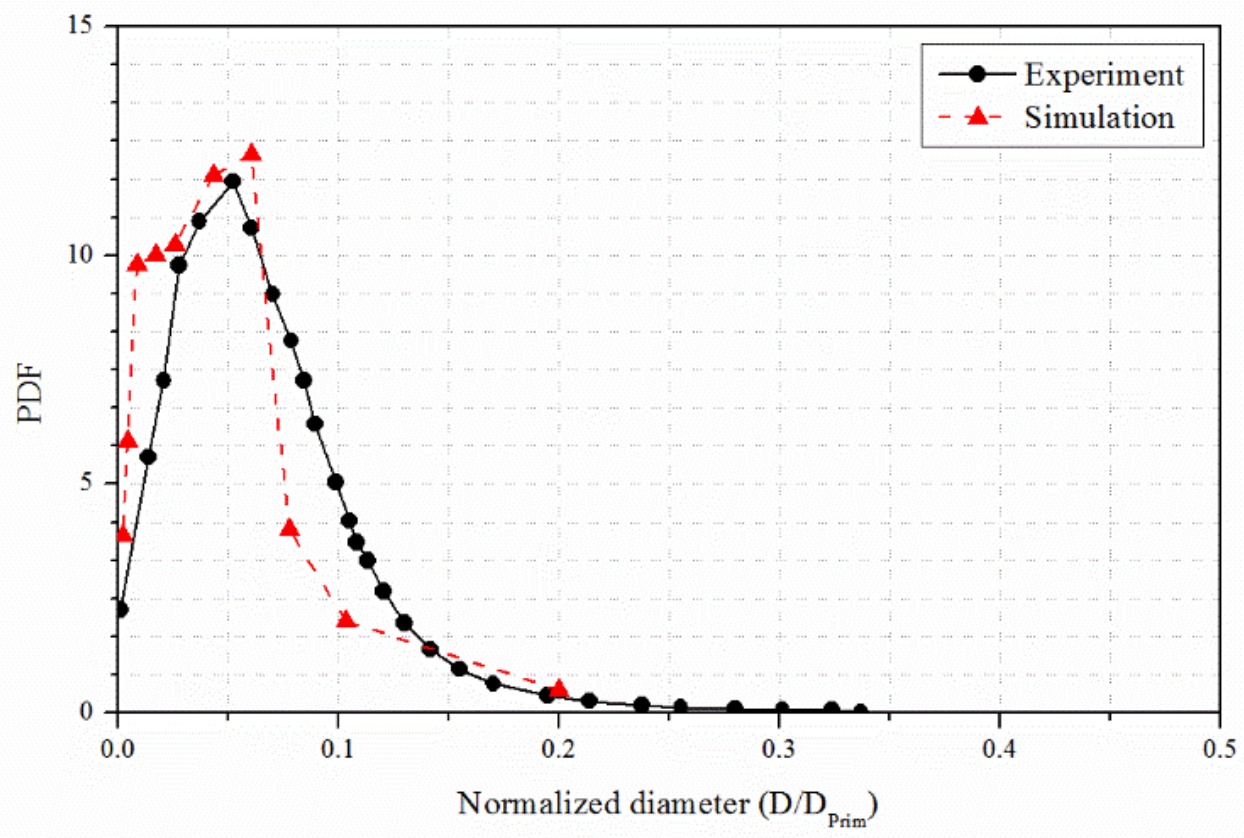

Figure 54. Comparison of size distribution of the splashed secondary droplets between simulation and experiment. The time that experiment data was taken was unknown.

\subsubsection{High-speed normal impact on a dry surface}

We simulate a water droplet impact on a dry solid surface at high impact speed. The water droplet has a diameter of $100 \mu \mathrm{m}$ and initial velocity of $100 \mathrm{~m} / \mathrm{s}$. The resulting $K$ value is approximately $1300, R e$ is 4000 , and $W e$ is 28000 . The condition is similar to droplet impact on an aircraft in operational condition. With the increase of the $K$ value, the splashed secondary droplets become smaller, which requires fine mesh. Grid sensitivity analysis indicates an effective grid resolution of 2800 cells per principle droplet diameter gives convergent results. In the simulation, a 6-level grid refinement is used. As shown in Figure $55(\mathrm{a})$, most of the splashed droplets have a diameter of about $0.3 \%$ of the primary droplet, and the total mass loss from splashing is $13.2 \%$ of the mass of the primary droplet. Compared with the previous case of $K=186.6$, the bandwidth of the size distribution 
function at $K=1300$ is much narrower. Figure 55(b) shows the velocity distribution of the splashed secondary droplets. The velocity of most secondary droplets ranges from approximately 100 to $250 \mathrm{~m} / \mathrm{s}$, which is much higher than initial impact velocity of 100 $\mathrm{m} / \mathrm{s}$. Figure 55(c) shows the shooting angle distribution of the secondary droplets. Most shooting angles fall in the range between 0 and $40^{\circ}$. Note that negative shooting angle means the secondary droplet from early stage of splashing is moving downward.
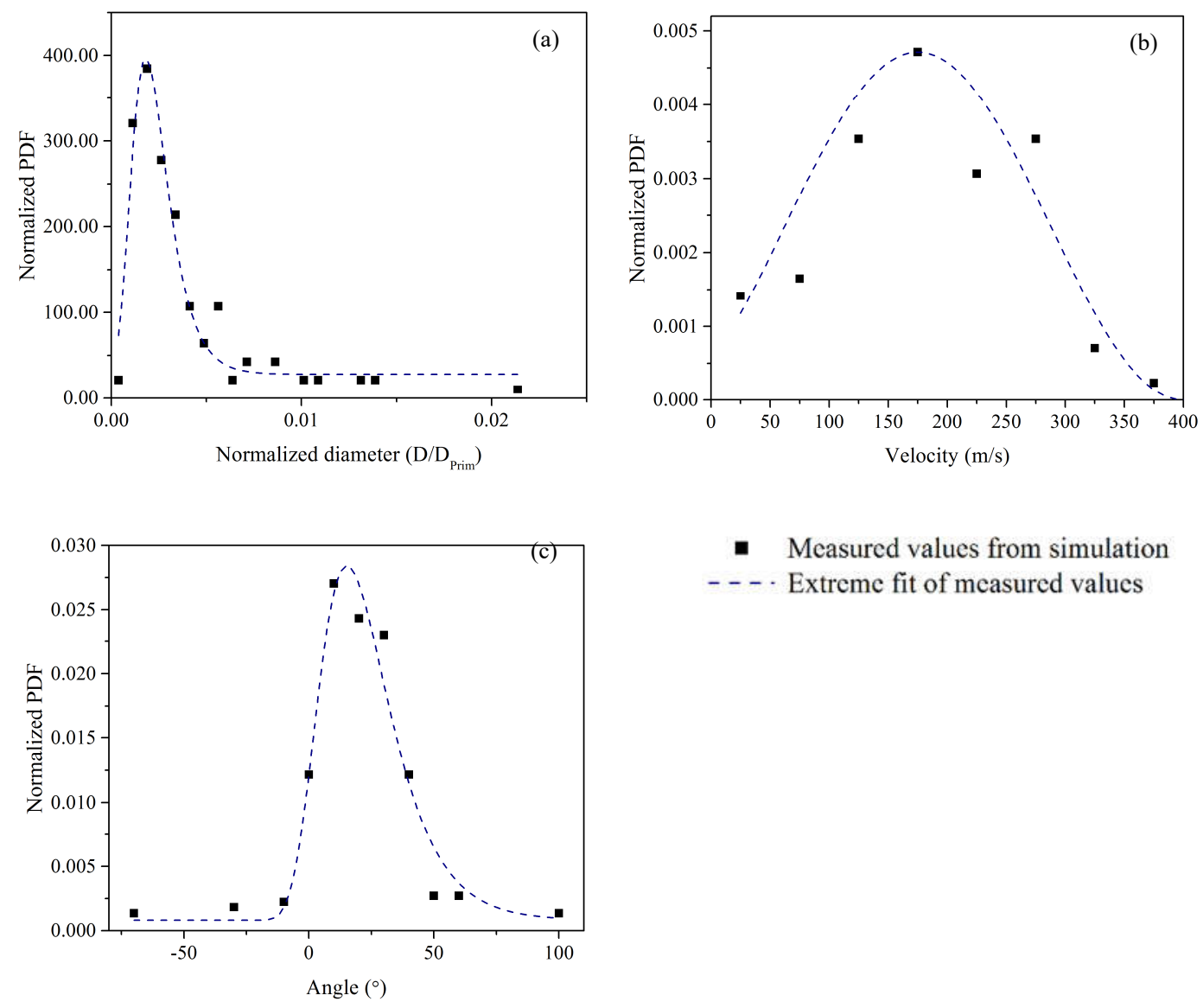

- Measured values from simulation - - . Extreme fit of measured values

Figure 55. Normalized distributions of secondary droplet diameter, velocity, and shooting angle for $\mathrm{K} \approx 1300$. 


\subsubsection{Droplet impact on thin liquid film}

During flight, wing surface is often covered with a liquid film from the impingement of previous droplets. Therefore, understanding the droplet impact on wet surface is crucial for water collection efficiency calculation. Experimental studies (Yarin \& Weiss, 1995; Cossali et al., 1997; Wang \& Chen, 2000; Rioboo et al., 2003) revealed the threshold for droplet splashing on thin liquid film. For a stream of droplet hitting the surface, Yarin and Weiss proposed the following threshold velocity for splashing:

$$
V_{0 S}=18\left(\frac{\sigma}{\rho}\right)^{1 / 4} v^{1 / 8} f^{3 / 8}
$$

where $f$ is the frequency of incoming droplets. For single droplet problem, $f$ can be replaced with $V_{0} / D_{0}$. Their study showed that the droplet diameter has no effect on the splashing threshold and the liquid film thickness is unimportant. Cossali et al. (Cossali et al., 1997) proposed the following threshold for droplet splashing on thin liquid film with consideration of film thickness:

$$
K_{S}=2100+5880\left(\frac{h}{D_{0}}\right)^{1.44} \quad \text { for } 0.1<\frac{h}{D_{0}}<1 \text { and } O h>0.007
$$

The threshold is valid for $h / D_{0}>0.1$. For $h / D_{0}<0.1$, Rioboo et al. (Rioboo et al., 2003) and Wang and Chen (Wang \& Chen, 2000) suggested a threshold of $K_{S} \approx 400$.

Zhang and $\mathrm{Hu}$ (Zhang \& Hu, 2014) experimentally studied the water film over a NACA 0012 wing and measured the thickness of the water film. For wind speed ranges from 10 $\mathrm{m} / \mathrm{s}$ to $30 \mathrm{~m} / \mathrm{s}$, the measured film thickness ranges from approximately $2 \mu \mathrm{m}$ to $40 \mu \mathrm{m}$, with 
thinner film for higher wing speed. The film thickness increases further downstream. For operational flight speed, which is much higher than $30 \mathrm{~m} / \mathrm{s}$, the liquid film is supposed to be thinner than $2 \mu \mathrm{m}$.

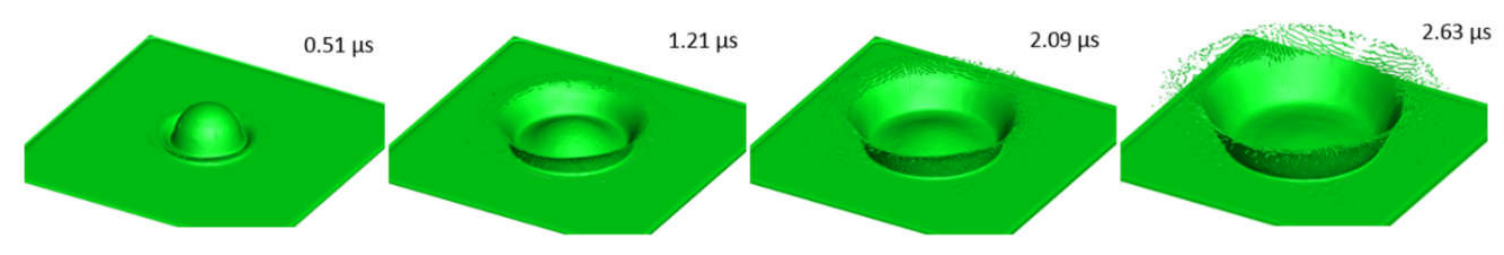

Figure 56. Droplet impingement on a thin water film. $D_{0}=100 \mu \mathrm{m}, h=5 \mu \mathrm{m}, V_{0}=78 \mathrm{~m} / \mathrm{s}$ and $K=667$.

Figure 56 shows the three-dimensional simulations of normal impact of a single water droplet onto a predefined thin water film. The initial diameter of the liquid droplet, $D_{0}$, is $100 \mu \mathrm{m}$, the normal impact velocity, $V_{0}$, is $78 \mathrm{~m} / \mathrm{s}$, and the film thickness, $h$, is $5 \mu \mathrm{m}$. The $K$ value is 667 , which is greater than the threshold value $K_{S}$ of 400 , and $V_{0}$ is greater than the threshold $V_{0 S}$ of $28.8 \mathrm{~m} / \mathrm{s}$. The equivalent grid size is 288 cells per initial droplet diameter with four-level adaptive mesh refinement. The simulation captures the formation and propagation of the lamellae. At the top of the lamella, the free rim becomes unstable and breaks up into multiple secondary droplets. The calculated mass loss from splashing of this case is $26.56 \%$, which is significantly higher than splashed mass (13.2\%) from the impact on a dry surface under otherwise same operating conditions. Because wing surface 
is wet during flight, the mass loss from wet surface splashing should be adopted for calculating water collecting efficiency.

\subsubsection{Code validation: water collection efficiency calculation for a sphere}

We validate the water collection efficiency calculated using our procedure with experimental measurement. The water collection efficiency calculation for a sphere body is performed experimentally by Lewis and Ruggeri (Lewis \& Ruggeri, 1957). The test body is a sphere of $15.04-\mathrm{cm}$ diameter, the MVD of spray generated by nozzles is $18.6 \mu \mathrm{m}$, and air speed is $80.8 \mathrm{~m} / \mathrm{s}$. Our three-dimensional simulation followed the same setup and is shown in Figure 57. One quarter of the sphere is simulated due to symmetry of the problem. Symmetric boundary conditions are imposed at the surfaces shown in red color, inflow boundary condition is imposed at the surface shown in blue color, and outflow boundary conditions are imposed at the other three surface. Mesh are only refined for the regions containing the droplet and three-level adaptive mesh refinement is used in this case. The equivalent grid resolution is 18 cells per droplet diameter. 

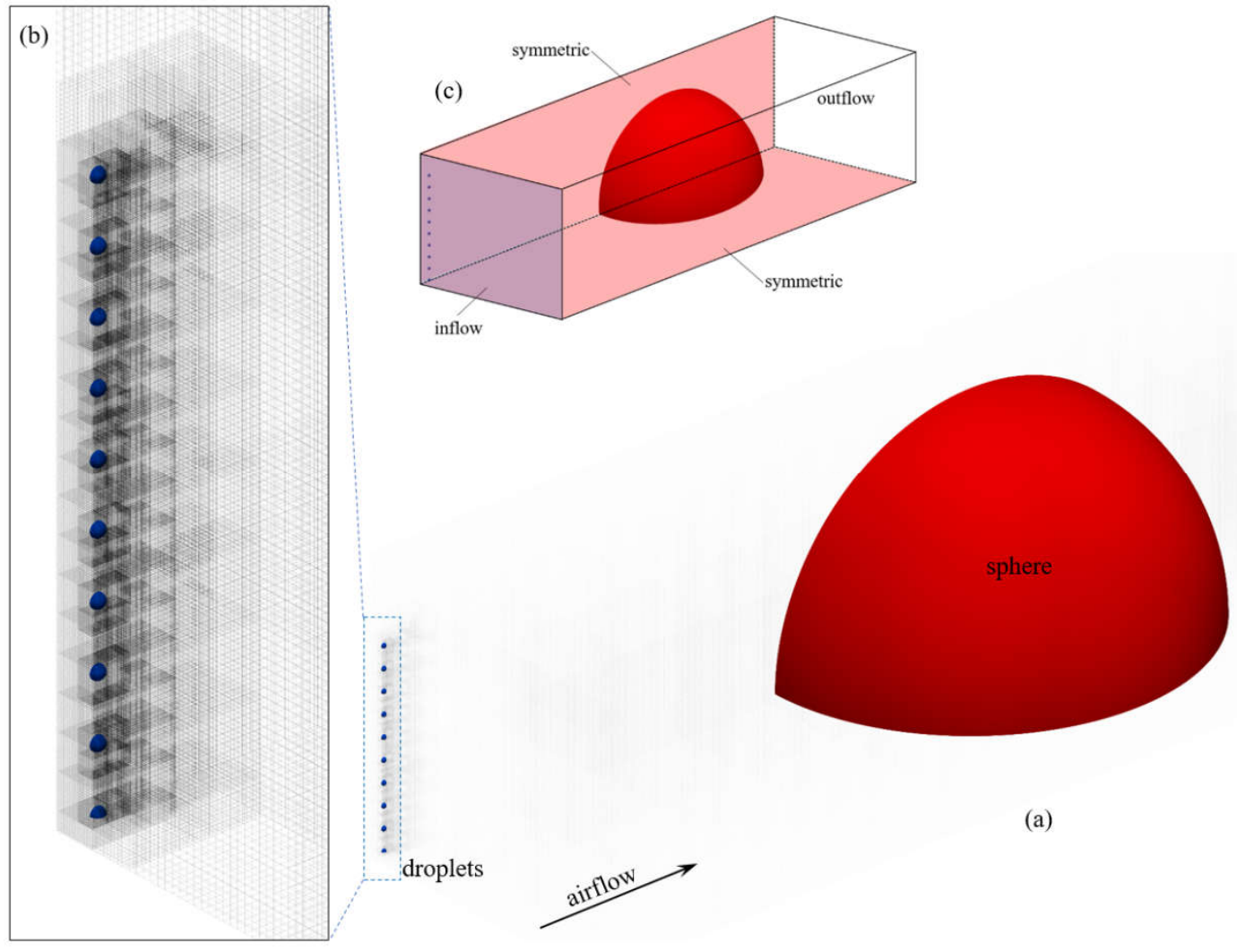

(a)

Figure 57. Three-dimensional simulation of droplets impact on a sphere. (a) Simulation setup. (b) zoomed-in view of droplets with three-level adaptive mesh refinement. (c) Boundary conditions: blue - inflow; red - symmetric; uncolored - outflow.

The water collection efficiency calculation follows the procedure described in Figure 52. In the first step, to obtain an MVD of $18.6 \mu \mathrm{m}$, a 7-bin droplet distribution is used as shown in Table 5. The droplets are positioned at locations where flow velocity is nearly parallel to the freestream velocity. When droplets contact the airfoil, the water collection efficiency is calculated by considering the impact angle. The impact limit is determined by the locations of the very top and bottom droplets impacting onto the airfoil. Figure 58 compares the numerical results of water collection efficiency with experimental data. Red triangles 
are collection efficiency without considering the splashing effect. Blue squares are collection efficiency with the consideration of the splashed mass. The numerical result of collection efficiency better matches experimental measurement when splashing is considered. The comparison results demonstrate that splashing phenomenon is critical in calculating collection efficiency.

Table 5. Discretized droplet distributions for MVD of $18.6 \mu \mathrm{m}$

\begin{tabular}{lccccccc}
\hline droplet size $(\mu \mathrm{m})$ & 5.77 & 9.67 & 13.21 & 18.6 & 25.48 & 32.36 & 41.29 \\
\hline $\begin{array}{l}\text { volume } \\
\text { percentage }\end{array}$ & $5 \%$ & $10 \%$ & $20 \%$ & $30 \%$ & $20 \%$ & $10 \%$ & $5 \%$ \\
\hline
\end{tabular}

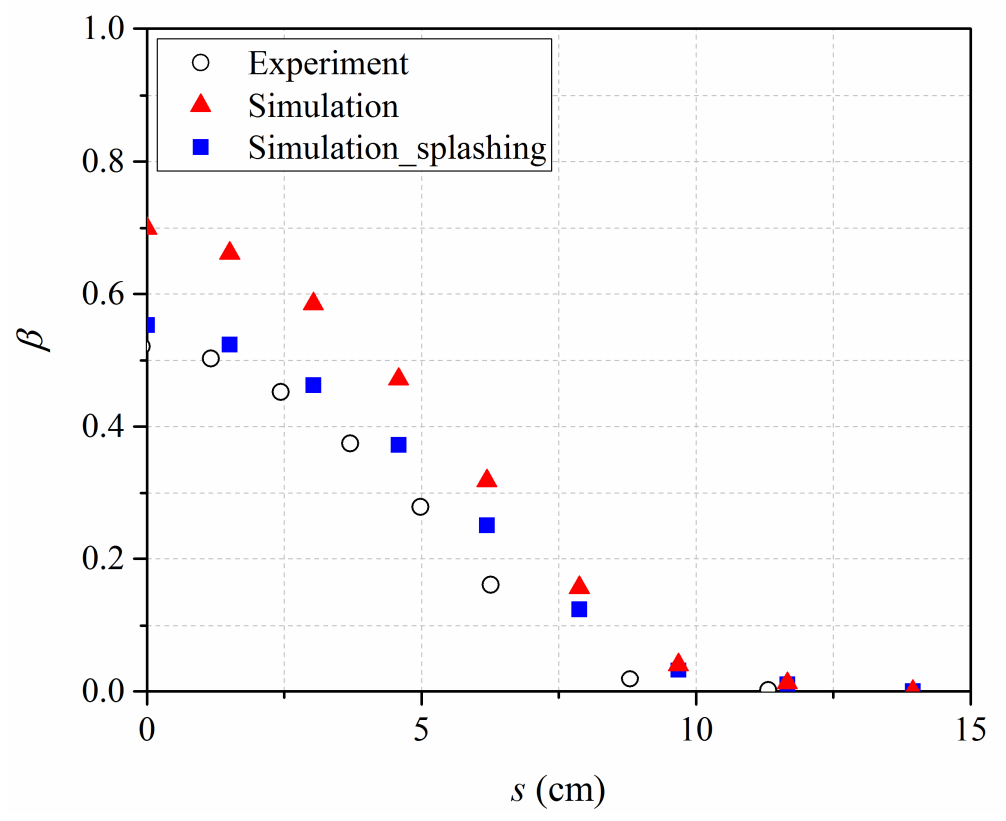

Figure 58. Water collection efficiency comparison between experiment and simulation. 


\subsubsection{Water collection efficiency calculation for an MS(1)-317 airfoil}

Water collection efficiency for an MS(1)-317 airfoil is investigated. The experiment was conducted by Tan (Tan, 2004). The airfoil chord is $0.914 \mathrm{~m}$, air speed is $78 \mathrm{~m} / \mathrm{s}$, AOA is $0^{\circ}$, and MVD is $92 \mu \mathrm{m}$. In the experiment, a dye-tracer technique was used to obtain the water collection efficiencies and impact limits (Gelder et al., 1956). Dyed water droplets were sprayed into the tunnel by nozzles and the surface of the airfoil was covered with blotter paper to absorb the dye. Our simulation follows the same setup and is shown in Figure 59. One narrow band of the airfoil is simulated and droplets are positioned at locations where flow velocity is nearly parallel to the freestream velocity. Mesh are only refined for the regions containing the droplet and two-level adaptive mesh refinement is used in this case. The equivalent grid resolution is 12 cells per droplet diameter. To obtain an MVD of $92 \mu \mathrm{m}$, a 7-bin droplet distribution is used as shown in Table 6, and 21 droplets

of the same size are used for each group as shown in Figure 59. Our analysis shows that further increase the number of droplets does not affect the collection efficiency. 


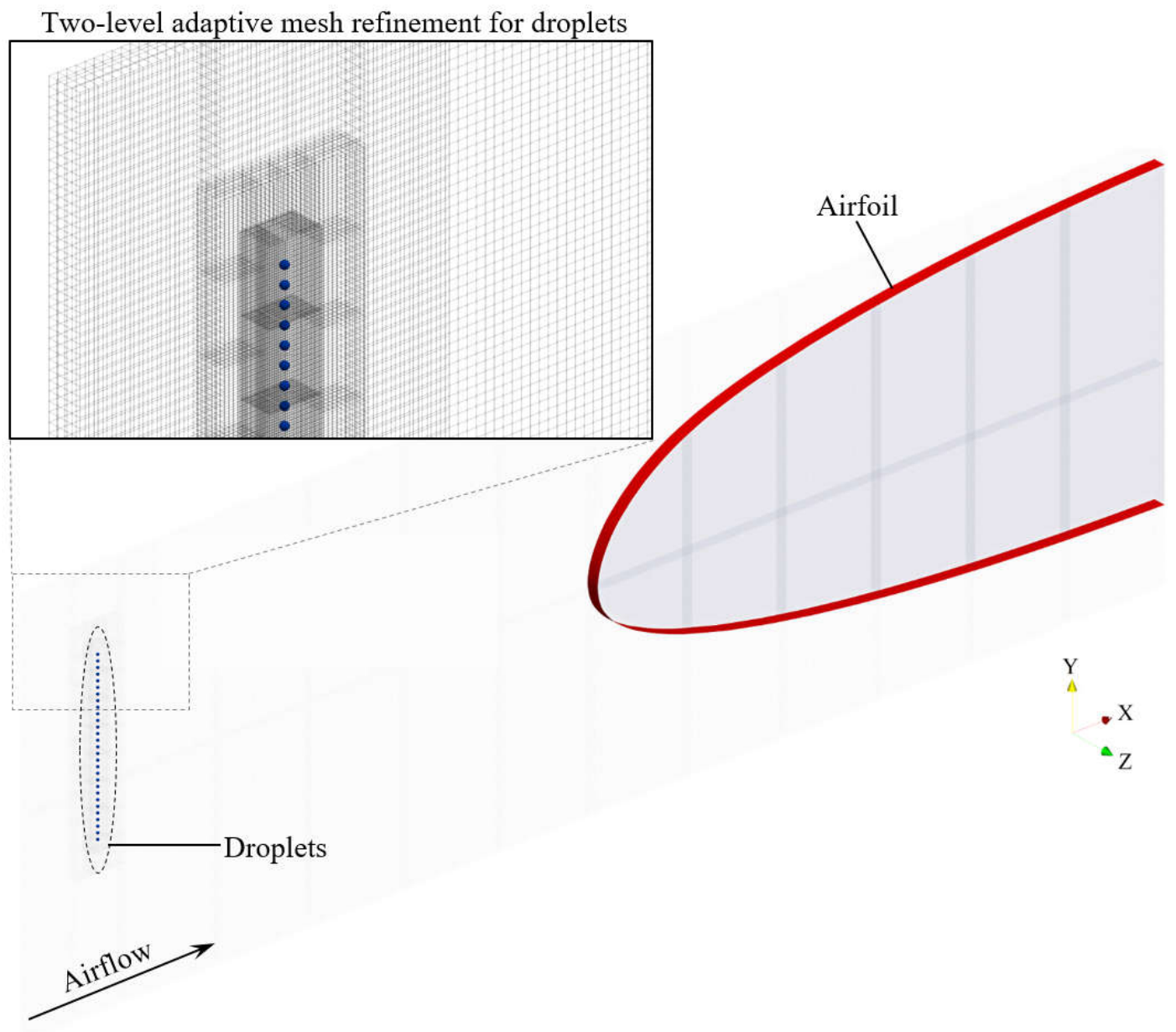

Figure 59. Three-dimensional simulation setup of droplets impact on an MS(1)-317 airfoil. The zoomed-in view shows the two-level adaptive mesh refinement near the regions containing droplets.

Table 6. Discretized droplet distributions for MVD of $92 \mu \mathrm{m}$

\begin{tabular}{lccccccc}
\hline droplet size $(\mu \mathrm{m})$ & 29.14 & 48.88 & 66.74 & 94.0 & 128.78 & 163.56 & 208.68 \\
\hline $\begin{array}{l}\text { volume } \\
\text { percentage }\end{array}$ & $5 \%$ & $10 \%$ & $20 \%$ & $30 \%$ & $20 \%$ & $10 \%$ & $5 \%$ \\
\hline
\end{tabular}


Figure 60 is the sliced view and shows the streamlines after droplets impact on the airfoil surface. Unlike the existing icing code such as LEWICE, the interaction between droplets and air is fully coupled in our simulations. As shown in Figure 60, the streamlines are clearly affected by the existence of droplets, and the droplets deform prior to impact due to the effect of surrounding air.

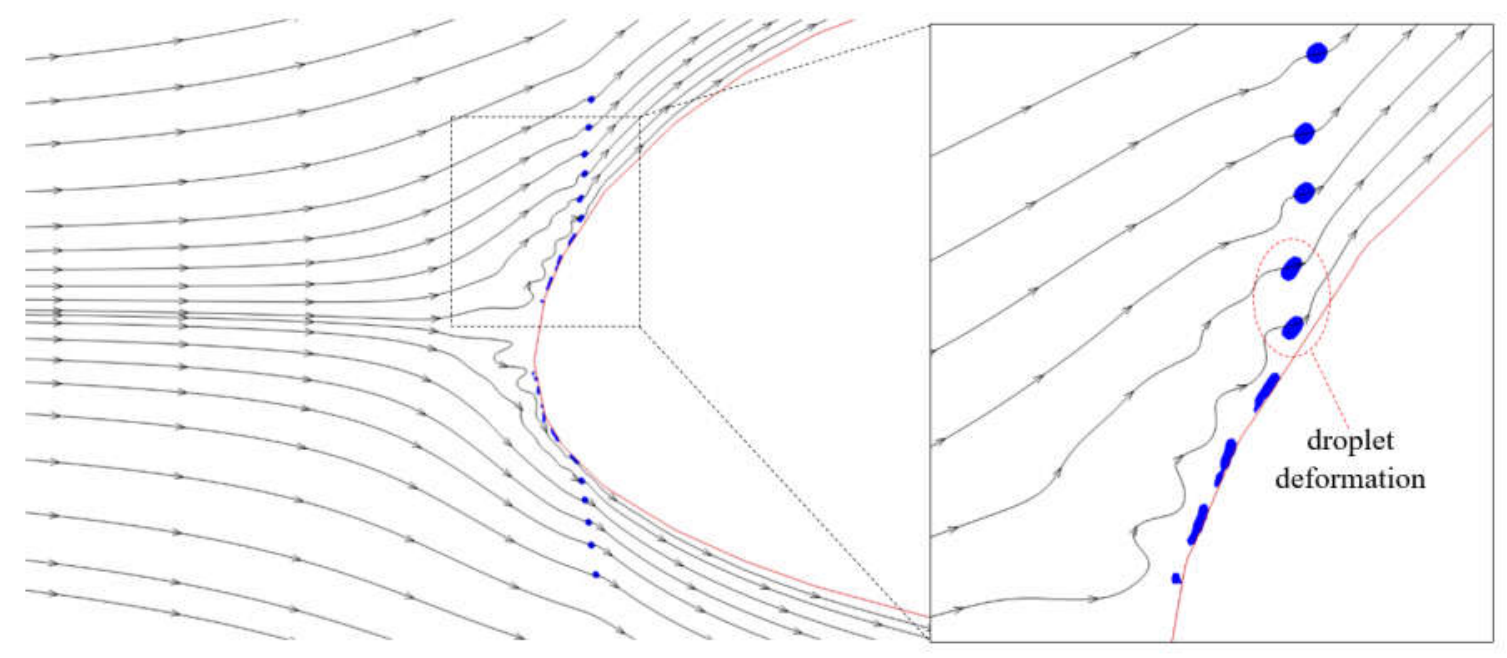

Figure 60. Altered streamlines and droplet deformation due to interaction between droplets and airflow.

The calculated water collection efficiency of each group is compared with LEWICE results in Figure 61. For the group of smallest droplets $(29.14 \mu \mathrm{m}$, cloud-sized droplets), our simulation closely matches LEWICE result. For large droplets, LEWICE shows higher limits than our simulation. LEWICE is validated for droplets whose sizes are between 5 $\mu \mathrm{m}$ and $50 \mu \mathrm{m}$ (Wright, 2008) and it assumes droplets are solid and spherical. The 
trajectory is calculated as a post-processing in which the flow affects the droplets but not the other way around. All these could contribute to the discrepancy between LEWICE results and our results when droplet sizes are relatively large. 

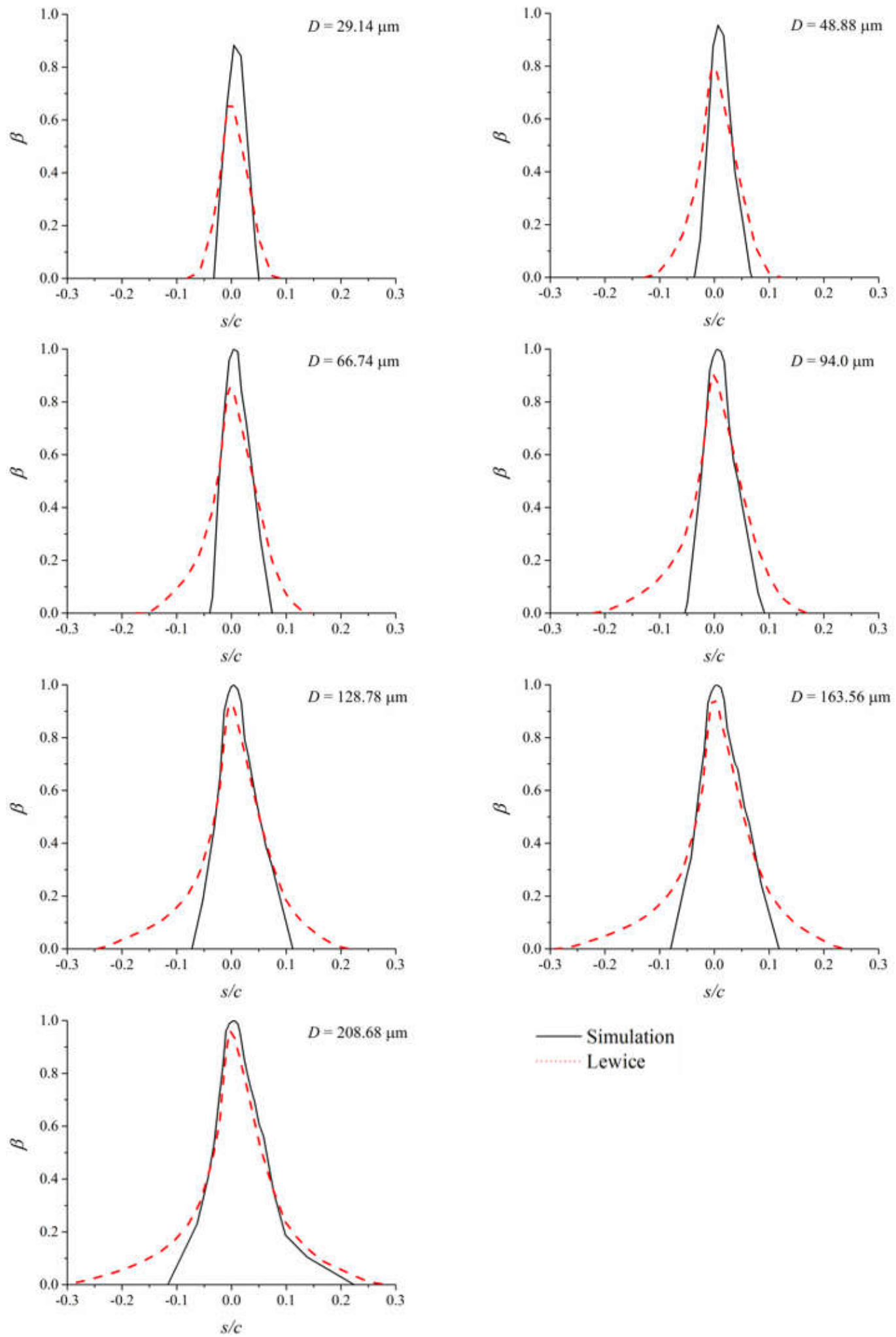

- Simulation Lewice

Figure 61. Water collection efficiencies of single-sized droplets. 
For those droplets that hit the wing, their water collection efficiency is further adjust with the consideration of splashing. Figure 62 shows the combined $\beta$ result with the volume percentage of each droplet size counted. The line with circles is the experimental data, the red dashed line is our simulation result without splashing effect, the blue line shows our simulation with splashing effect considered, and the black dotted line is LEWICE result. The comparison clearly shows that LEWICE overestimates the impact limit, while our simulations with or without splashing effect fit the experimental impact limit. Because droplet splashing is only occurring near the leading edge area, splashing effect is negligible for large impact angle.

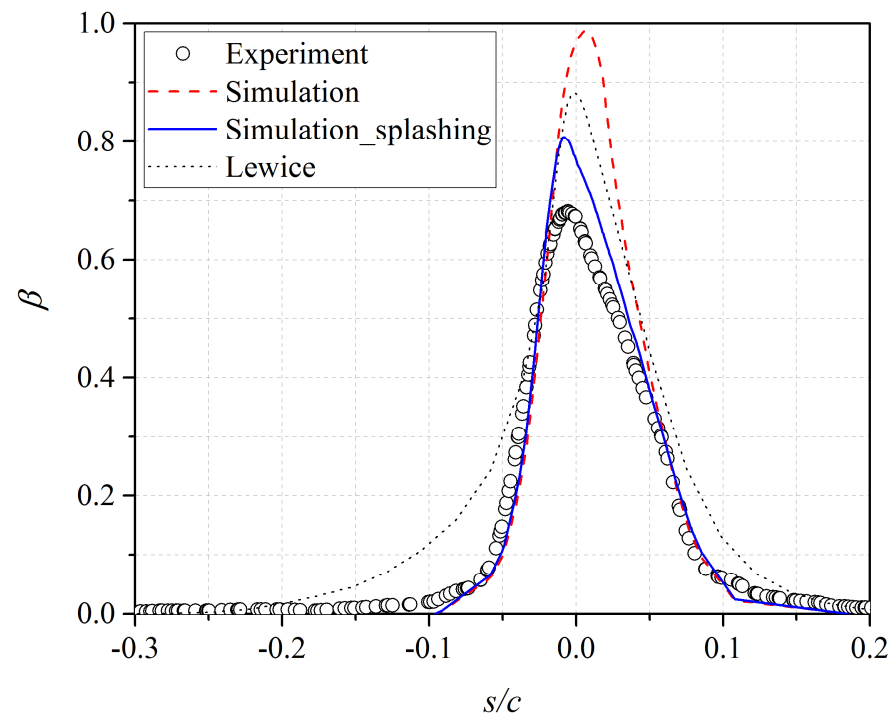

Figure 62. Water collection efficiencies comparison of experiment, simulations, and LEWICE. 


\subsection{Conclusions}

A numerical study was conducted to investigate droplet impingement phenomenon on dry and wet surfaces and to calculate collection efficiency of water droplets on an MS(1)-317 wing. The flow field around the airfoil was described by solving Navier-Stokes equations. The droplet impact on the surface and the subsequent phenomenon were captured directly in our simulations. The adaptive mesh refinement technique was employed to refine mesh near regions of interest. The numerical code was first validated against experiment of drop impact on a dry surface and good agreement was achieved. Simulations of droplets impact on dry and wet surfaces were performed to study mass loss from droplet splashing effect. Another validation case was conducted to calculate water collection efficiency of a sphere body and our numerical result agreed well with experimental data. Last, we calculated the water collection efficiency of an MS(1)-317 wing. Compared with experimental data, our simulation shows better agreement than LEWICE. LEWICE predicts well for cloud-sized droplet, but overestimates the impact limit for SLDs.

The following important conclusions can be made from the present study: (i) Droplet splashing leads to mass loss after impact, and splashing on thin liquid film is found to have significantly more mass loss than on dry surface. Neglecting droplet splashing effect may cause poor collection efficiency prediction. (ii) Fully coupling of droplets and airflow gives better prediction for droplet trajectories. The streamlines of airflow are altered by droplets, and the droplets deform due to the effect of surrounding air. 


\section{CHAPTER 7. GENERAL CONCLUSIONS}

In the present study, the drop impingement on dry and wet surfaces were numerically investigated. The Navier-Stokes equations were solved using the projection method on Cartesian grids and the moment-of-fluid method was used to construct the interfaces. The numerical results showed that the moment-of-fluid method could accurately capture the interfaces. The numerical method was validated with experimental results and theoretical predictions and good agreement was achieved qualitatively and quantitatively.

The underlying physics of drop impingement phenomenon were elucidated to improve the understanding of drop splashing mechanism. The present study revealed that a thin film was formed after the droplet contacted the solid surface. The thin film moved at a low speed at the contact with the solid due to viscous effect while the film moved at a high speed away from the solid. The splashing is due to the aerodynamic forces on the thin film. For thin film splashing, the lamella is initiated from the preexisting liquid film on solid surface and the liquid content from the droplet sliding along above it.

The study of ambient air effect showed that the dry surface splashing was attenuated when the air density was lowered, however, the thin film splashing was found to be not affected by the ambient air prominently but had much larger mass loss compared with dry surface splashing. 
For drop oblique impingement on a liquid layer, the tangential impact velocity, the liquid film thickness and the drop-film density ratio all have significant effects on the impact outcome. Furthermore, when multiple drops impact on a liquid layer, strong interactions between the lamellae formed after impact were observed.

Drop impingement phenomenon, especially splashing, is directly related to aircraft icing under supercooled large droplet (SLD) condition. Using the well-validated numerical method, a new approach was proposed to calculate water collection efficiency, one of the most important stages in aircraft icing analysis. Compared with experimental data, the numerical results showed better agreement than LEWICE, which predicted well for cloudsized droplet, but overestimated the impact limit for SLDs. The simulations revealed that splashing leads to mass loss after impact and neglecting droplet splashing effect may cause poor collection efficiency prediction. 


\section{REFERENCES}

Administration, F. A. (2014). Airworthiness Standards: Transport Category Airplanes FAR 25 (Amendment 140).

Agency, E. A. S. (2015). Certification Specifications and Acceptable Means of Compliance for Large Aeroplanes CS-25 (Amendment 16).

Ahn, H. T. and M. Shashkov. (2007). Multi-material interface reconstruction on generalized polyhedral meshes. Journal of Computational Physics 226(2): 2096-2132.

Ahn, H. T. and M. Shashkov. (2009). Adaptive moment-of-fluid method. Journal of Computational Physics 228(8): 2792-2821.

Allen, R. F. (1975). Role of Surface-Tension in Splashing. Journal of Colloid and Interface Science 51(2): 350-351.

Azimi, G., R. Dhiman, H. M. Kwon, A. T. Paxson and K. K. Varanasi. (2013). Hydrophobicity of rare-earth oxide ceramics. Nat. Mater. 12(4): 315-320.

Barnes, H., Y. Hardalupas, A. Taylor and J. Wilkins (1999). An investigation of the interaction between two adjacent droplets, ILASS-Europe.

Bilanin, A. J. (1991). Proposed Modifications to Ice Accretion Icing Scaling Theory. Journal of Aircraft 28(6): 353-359.

Bird, J. C., S. S. Tsai and H. A. Stone. (2009). Inclined to splash: triggering and inhibiting a splash with tangential velocity. New Journal of Physics 11(6): 063017.

Blake, J., D. Thompson, T. Strobl, E. Bonaccurso and D. Raps. (2014). Effects of Surface Characteristics and Droplet Diameter on the Freezing of Supercooled Water Droplets Impacting a Cooled Substrate. 6th AIAA Atmospheric and Space Environments Conference. 
Bond, T., M. Potapczuk and D. Miller. (2003). Overview of SLD engineering tool development. AIAA Paper 386: 41.

Bourgault, Y., W. G. Habashi, J. Dompierre and G. S. Baruzzi. (1999). A finite element method study of Eulerian droplets impingement models. International Journal for Numerical Methods in Fluids 29(4): 429-449.

Brackbill, J., D. B. Kothe and C. Zemach. (1992). A continuum method for modeling surface tension. Journal of Computational Physics 100(2): 335-354.

Bragg, M. B. (1996). Aerodynamics of supercooled- large-droplet ice accretions and the effect on aircraft control. Proceedings of the FAA International Conference on Aircraft Inflight Icing, Springfield, VA.

Bragg, M. B. (1996). Aircraft Aerodynamic Effects due to Large Droplet Ice Accretions. AIAA Paper 0932.

Bragg, M. B., G. M. Gregorek and J. D. Lee. (1986). Airfoil Aerodynamics in Icing Conditions. Journal of Aircraft 23(1): 76-81.

Bussmann, M., S. Chandra and J. Mostaghimi. (2000). Modeling the splash of a droplet impacting a solid surface. Physics of Fluids 12: 3121.

Bussmann, M., J. Mostaghimi and S. Chandra. (1999). On a three-dimensional volume tracking model of droplet impact. Physics of Fluids 11: 1406.

Chandra, S. and C. Avedisian. (1991). On the collision of a droplet with a solid surface. Proceedings: Mathematical and Physical Sciences: 13-41.

Cheng, M. and J. Lou. (2015). A numerical study on splash Of oblique drop impact on wet walls. Computers \& Fluids 115: 11-24.

Ching, B., M. W. Golay and T. J. Johnson. (1984). Droplet Impacts Upon Liquid Surfaces. Science 226(4674): 535-537. 
Cossali, G. E., A. Coghe and M. Marengo. (1997). The impact of a single drop on a wetted solid surface. Experiments in Fluids 22(6): 463-472.

Cossali, G. E., M. Marengo, A. Coghe and S. Zhdanov. (2004). The role of time in single drop splash on thin film. Experiments in Fluids 36(6): 888-900.

Criscione, A., R. Röhrig, L. Opfer, I. Roisman and S. Jakirlic. (2011). Numerical investigation of impacting water drops in air cross-flow.

Da Silveira, R. A., C. R. Maliska, D. A. Estivam and R. Mendes. (2003). Evaluation of Collection Efficiency Methods for Icing Analysis. Proceedings of 17th International Congress of Mechanical Engineering.

Deegan, R. D., P. Brunet and J. Eggers. (2008). Complexities of splashing. Nonlinearity 21(1): C1-C11.

Dunn, T. A., E. Loth and M. B. Bragg. (1999). Computational investigation of simulated large-droplet ice shapes on airfoil aerodynamics. Journal of Aircraft 36(5): 836-843.

Dyadechko, V. and M. Shashkov. (2005). Moment-of-fluid interface reconstruction. Los Alamos report LA-UR-05-7571.

Dyadechko, V. and M. Shashkov. (2008). Reconstruction of multi-material interfaces from moment data. Journal of Computational Physics 227(11): 5361-5384.

Footte, G. B. (1975). The water drop rebound problem: Dynamics of collision. Journal of the Atmospheric Sciences 32(2): 390-402.

Fukai, J., Y. Shiiba, T. Yamamoto, O. Miyatake, D. Poulikakos, C. Megaridis and Z. Zhao. (1995). Wetting effects on the spreading of a liquid droplet colliding with a flat surface: experiment and modeling. Physics of Fluids 7: 236.

Fullana, J. M. and S. Zaleski. (1999). Stability of a growing end rim in a liquid sheet of uniform thickness. Physics of Fluids 11(5): 952-954. 
Gelder, T. F., W. H. Smyers and U. v. Glahn. (1956). Experimental Droplet Impingement on Several Two-Dimensional Airfoils with Thickness Ratios of 6 to 16 Percent. Cleveland, Ohio, Lewis Flight Propulsion Laboratory. Technical Note 3839.

Gent, J. F. and R. Moser. (2003). D. Miller,"Results from SLD Mass Loss Tests in the ACT Luton Icing Research Wind Tunnel,” AIAA-2003-389, Jan. 6-9.

Gibou, F., R. Fedkiw, R. Caflisch and S. Osher. (2003). A level set approach for the numerical simulation of dendritic growth. Journal of Scientific Computing 19(1-3): 183199.

Guo, Y. and Y. Lian. (2017). High-speed oblique drop impact on thin liquid films. Physics of Fluids 29(8).

Guo, Y., Y. Lian and M. Sussman. (2016). Investigation of drop impact on dry and wet surfaces with consideration of surrounding air. Physics of Fluids 28(7): 073303.

Haller, K., D. Poulikakos, Y. Ventikos and P. Monkewitz. (2003). Shock wave formation in droplet impact on a rigid surface: lateral liquid motion and multiple wave structure in the contact line region. Journal of Fluid Mechanics 490: 1-14.

Hoffman, R. L. (1975). Study of Advancing Interface .1. Interface Shape in Liquid-Gas Systems. Journal of Colloid and Interface Science 50(2): 228-241.

Honsek, R., W. Habashi and M. Aube. (2008). Eulerian Modeling of In-flight Icing Due to Supercooled Large Droplets. JOURNAL OF AIRCRAFT 45(4).

Honsek, R., W. G. Habashi and M. S. Aubé. (2008). Eulerian Modeling of In-Flight Icing Due to Supercooled Large Droplets. JOURNAL OF AIRCRAFT 45(4).

Isaac, G., S. Cober, J. Strapp, D. Hudak, T. Ratvasky, D. Marcotte and F. Fabry (2001). Preliminary results from the Alliance Icing Research Study (AIRS). AIAA 39th Aerospace Sci. Meeting and Exhibit.

Jayaratne, O. and B. Mason. (1964). The coalescence and bouncing of water drops at an air/water interface. Proceedings of the Royal Society of London. Series A. Mathematical and Physical Sciences 280(1383): 545-565. 
Jemison, M., E. Loch, M. Sussman, M. Shashkov, M. Arienti, M. Ohta and Y. Wang. (2012). A Coupled Level Set-Moment of Fluid Method for Incompressible Two-Phase Flows. Journal of Scientific Computing: 1-38.

Jemison, M., M. Sussman and M. Arienti. (2013). Compressible, Multiphase Semi-Implicit Method with Moment of Fluid Interface Representation. Submitted to Journal of Computational Physics.

Jemison, M., M. Sussman and M. Arienti. (2014). Compressible, multiphase semi-implicit method with moment of fluid interface representation. Journal of Computational Physics 279: $182-217$.

Jiang, T.-S., S.-G. Oh and J. C. Slattery. (1978). Correlation for Dynamic Contact Angle. Journal of Colloid and Interface Science 69(1).

Jiang, T.-S., O. Soo-Gun and J. C. Slattery. (1979). Correlation for dynamic contact angle. Journal of Colloid and Interface Science 69(1): 74-77.

Josserand, C. and S. T. Thoroddsen. (2016). Drop Impact on a Solid Surface. Annual Review of Fluid Mechanics, Vol 48 48: 365-391.

Josserand, C. and S. Zaleski. (2003). Droplet splashing on a thin liquid film. Physics of Fluids 15(6): 1650-1657.

Jung, S. and R. Myong Numerical Modeling for Eulerian Droplet Impingement in Supercooled Large Droplet Conditions. 51st AIAA Aerospace Sciences Meeting including the New Horizons Forum and Aerospace Exposition.

Kim, H. Y. and J. H. Chun. (2001). The recoiling of liquid droplets upon collision with solid surfaces. Physics of Fluids 13(3): 643-659.

Kistler, S. (1993). Hydrodynamics of wetting. Wettability 6: 311-430.

Kolinski, J. M., S. M. Rubinstein, S. Mandre, M. P. Brenner, D. A. Weitz and L. Mahadevan. (2012). Skating on a film of air: drops impacting on a surface. Physical review letters 108(7): 074503. 
Krechetnikov, R. and G. M. Homsy. (2009). Crown-forming instability phenomena in the drop splash problem. Journal of Colloid and Interface Science 331(2): 555-559.

Kwatra, N., J. Su, J. T. Grétarsson and R. Fedkiw. (2009). A method for avoiding the acoustic time step restriction in compressible flow. Journal of Computational Physics 228(11): 4146-4161.

Latka, A., A. Strandburg-Peshkin, M. M. Driscoll, C. S. Stevens and S. R. Nagel. (2012). Creation of prompt and thin-sheet splashing by varying surface roughness or increasing air pressure. Physical review letters 109(5): 054501.

Lee, S. and M. B. Bragg. (1999). Experimental investigation of simulated large-droplet ice shapes on airfoil aerodynamics. Journal of Aircraft 36(5): 844-850.

Leneweit, G., R. Koehler, K. G. Roesner and G. Schafer. (2005). Regimes of drop morphology in oblique impact on deep fluids. Journal of Fluid Mechanics 543: 303-331.

Lesser, M. and J. Field. (1983). The impact of compressible liquids. Annual review of fluid mechanics 15(1): 97-122.

Lewis, J. P. and R. S. Ruggeri. (1957). Experimental Droplet Impingement on Four Bodies of Revolution.

Li, G. B., Y. S. Lian, Y. S. Guo, M. Jemison, M. Sussman, T. Helms and M. Arienti. (2015). Incompressible multiphase flow and encapsulation simulations using the moment-of-fluid method. International Journal for Numerical Methods in Fluids 79(9): 456-490.

Li, L., X. Jia, Y. Liu and M. Su. (2016). Simulation of double droplets impact on liquid film by a simplified lattice Boltzmann model. Applied Thermal Engineering 98: 656-669.

Lian, Y. S., Y. S. Guo and A. Work. (2014). Numerical Simulation of Supercooled Large Droplets Using the Moment of Fluid Method.

Lian, Y. S., Y. S. Guo and A. Work. (2014). Study of supercooled large droplet impacts using the moment of fluid method. 52nd AIAA Aerospace Sciences Meeting. 
Liang, G. T., Y. L. Guo, Y. Yang, N. Zhen and S. Q. Shen. (2013). Spreading and splashing during a single drop impact on an inclined wetted surface. Acta Mechanica 224(12): 29933004.

Lissaman, P. (1983). Low-Reynolds-number airfoils. Annual review of fluid mechanics 15(1): 223-239.

Liu, J., H. Vu, S. S. Yoon, R. Jepsen and G. Aguilar. (2010). Splashing Phenomena during Liquid Droplet Impact. Atomization and Sprays 20(4): 297-310.

Makkonen, L., T. Laakso, M. Marjaniemi and K. J. Finstad. (2001). Modeling ice accretion on wind turbines. Wind Engineering 3-21.

Mandre, S. and M. P. Brenner. (2012). The mechanism of a splash on a dry solid surface. Journal of Fluid Mechanics 690: 148-172.

Mani, M., S. Mandre and M. P. Brenner. (2010). Events before droplet splashing on a solid surface. Journal of Fluid Mechanics 647: 163--185.

Mao, T., D. Kuhn and H. Tran. (2004). Spread and rebound of liquid droplets upon impact on flat surfaces. AIChE Journal 43(9): 2169-2179.

Marengo, M. and C. Tropea. Zwischenbericht zum Forschungsvorhaben Aufprall von Tropfen auf Flusssigkeitsfilme. $\operatorname{Tr}$ 194(10): 1.

Mongruel, A., V. Daru, F. Feuillebois and S. Tabakova. (2009). Early post-impact time dynamics of viscous drops onto a solid dry surface. Physics of Fluids (1994-present) 21(3): 032101.

Morency, F., H. Beaugendre, G. S. Baruzzi and W. G. Habashi. (2001). FENSAP-ICE: A Comprehensive 3D Simulation Tool for In-flight Icing. AIAA Paper 2566.

Morency, F., F. Tezok and I. Paraschivoiu. (1999). Anti-icing system simulation using CANICE. Journal of Aircraft 36(6): 999-1006. 
Motzkus, C., F. Gensdarmes and E. Gehin. (2009). Parameter study of microdroplet formation by impact of millimetre-size droplets onto a liquid film. Journal of Aerosol Science 40(8): 680-692.

Mukherjee, S. and J. Abraham. (2007). Crown behavior in drop impact on wet walls. Physics of Fluids 19(5).

Mundo, C., M. Sommerfeld and C. Tropea. (1995). Droplet-wall collisions: experimental studies of the deformation and breakup process. International journal of multiphase flow 21(2): 151-173.

Mundo, C., C. Tropea and M. Sommerfeld. (1997). Numerical and experimental investigation of spray characteristics in the vicinity of a rigid wall. Experimental thermal and fluid science 15(3): 228-237.

Myers, T. G. and D. W. Hammond. (1999). Ice and water film growth from incoming supercooled droplets. International Journal of Heat and Mass Transfer 42(12): 2233-2242.

Nikolopoulos, N., A. Theodorakakos and G. Bergeles. (2005). Normal impingement of a droplet onto a wall film: a numerical investigation. International journal of heat and fluid flow 26(1): 119-132.

Nikolopoulos, N., A. Theodorakakos and G. Bergeles. (2007). Three-dimensional numerical investigation of a droplet impinging normally onto a wall film. Journal of Computational Physics 225(1): 322-341.

Okawa, T., T. Shiraishi and T. Mori. (2008). Effect of impingement angle on the outcome of single water drop impact onto a plane water surface. Experiments in Fluids 44(2): 331339.

Papadakis, M., M. Breer, N. Craig and X. Liu. (1994). Experimental Water Droplet Impingement Data on Airfoils, Simulated Ice Shapes, an Engine Inlet and a Finite Wing, NASA CR 4636, DOT/FAA/CT-TN93/18.

Papadakis, M., K. E. Hung, G. T. Vu, H. W. Yeong, C. S. Bidwell, M. D. Breer and T. J. Bencic. (2002). Experimental Investigation of Water Droplet Impingement on Airfoils, Finite Wings, and an S-Duct Engine Inlet, NASA TM 2002-211700. 
Papadakis, M., E. R., G. A. J. Freund, M. Breer, G. W. Zumwalt and L. Whitmer. (1989). An Experimental Method for Measuring Water Droplet Impingement Efficiency on Twoand Three-Dimensional Bodies, NASA Contractor Report 4257, DOT/FAA/CT-87/22.

Pasandideh-Fard, M., S. Aziz, S. Chandra and J. Mostaghimi. (2001). Cooling effectiveness of a water drop impinging on a hot surface. International journal of heat and fluid flow 22(2): 201-210.

Petty, K. R. and C. D. J. Floyd. (2004). A statistical review of aviation airframe icing accidents in the US. Proceedings of the 11th Conference on Aviation, Range, and Aerospace Hyannis.

Politovich, M. K. (1989). Aircraft Icing Caused by Large Supercooled Drops. Journal of Applied Meteorology 28(9): 856-868.

Potapczuk, M. G., K. M. Al-Khalil and M. T. Velazquez. (1993). Ice accretion and performance degradation calculations with LEWICE.

Raman, K., R. Jaiman, T. Lee and H. Low. (2015). On the dynamics of crown structure in simultaneous two droplets impact onto stationary and moving liquid film. Computers \& Fluids 107: 285-300.

Ranaudo, R. J., J. G. Batterson, A. L. Reehorst, T. H. Bond and T. M. Omara. (1991). Effects of Horizontal Tail Ice on Longitudinal Aerodynamic Derivatives. Journal of Aircraft 28(3): 193-199.

Rein, M. (1993). Phenomena of Liquid-Drop Impact on Solid and Liquid Surfaces. Fluid Dynamics Research 12(2): 61-93.

Rein, M. (1993). Phenomena of liquid drop impact on solid and liquid surfaces. Fluid Dynamics Research 12(2): 61-93.

Riboux, G. and J. M. Gordillo. (2014). Experiments of Drops Impacting a Smooth Solid Surface: A Model of the Critical Impact Speed for Drop Splashing. Physical Review Letters 113(2).

Riboux, G. and J. M. Gordillo. (2015). The diameters and velocities of the droplets ejected after splashing. J. Fluid Mech. 772: 630-648. 
Rieber, M. and A. Frohn. (1999). A numerical study on the mechanism of splashing. International journal of heat and fluid flow 20(5): 455-461.

Rioboo, R., C. Bauthier, J. Conti, M. Voue and J. De Coninck. (2003). Experimental investigation of splash and crown formation during single drop impact on wetted surfaces. Experiments in Fluids 35(6): 648-652.

Rioboo, R., C. Tropea and M. Marengo. (2001). Outcomes from a drop impact on solid surfaces. Atomization and Sprays 11(2): 155-165.

Roisman, I., B. Prunet-Foch, C. Tropea and M. Vignes-Adler. (2002). Multiple drop impact onto a dry solid substrate. Journal of Colloid and Interface Science 256(2): 396-410.

Roisman, I. V., K. Horvat and C. Tropea. (2006). Spray impact: Rim transverse instability initiating fingering and splash, and description of a secondary spray. Physics of Fluids 18(10).

Roisman, I. V., R. Rioboo, C. Tropea, I. V. Roisman, R. Rioboo and C. Tropea. (2002). Normal impact of a liquid drop on a dry surface: model for spreading and receding. Proceedings of the Royal Society of London. Series A: Mathematical, Physical and Engineering Sciences 458(2022): 1411-1430.

Roisman, I. V. and C. Tropea. (2002). Impact of a drop onto a wetted wall: description of crown formation and propagation. Journal of Fluid Mechanics 472: 373-397.

Rutkowski, A., W. Wright and M. Potapczuk. (2003). Numerical Study of Droplet Splashing and Re-Impingement. AIAA paper 388.

Samenfink, W., A. Elsasser, K. Dullenkopf and S. Wittig. (1999). Droplet interaction with shear-driven liquid films: analysis of deposition and secondary droplet characteristics. International journal of heat and fluid flow 20(5): 462-469.

Samenfink, W., A. Elsäßer, K. Dullenkopf and S. Wittig. (1999). Droplet interaction with shear-driven liquid films: analysis of deposition and secondary droplet characteristics. International journal of heat and fluid flow 20(5): 462-469. 
Schmehl, R., H. Rosskamp, M. Willmann and S. Wittig. (1999). CFD analysis of spray propagation and evaporation including wall film formation and spray/film interactions. International journal of heat and fluid flow 20(5): 520-529.

Schofield, S. P., R. V. Garimella, M. M. Francois and R. Loubere. (2008). Material orderindependent interface reconstruction using power diagrams. International Journal for Numerical Methods in Fluids 56(6): 643-659.

Schroll, R. D., C. Josserand, S. Zaleski and W. W. Zhang. (2010). Impact of a viscous liquid drop. Physical review letters 104(3): 034504.

Shetabivash, H., F. Ommi and G. Heidarinejad. (2014). Numerical analysis of droplet impact onto liquid film. Physics of Fluids 26(1).

Šikalo, Š., H. D. Wilhelm, I. Roisman, S. Jakirlić and C. Tropea. (2005). Dynamic contact angle of spreading droplets: Experiments and simulations. Physics of Fluids 17: 062103.

Silva, D. M. d., N. Bachchan, I. Kim and O. Peroomian. (2014). Icing Collection Efficiency Prediction using an Eulerian-Eulerian Approach. 44th AIAA Fluid Dynamics Conference.

Stanton, D. W. and C. J. Rutland. (1998). Multi-dimensional modeling of thin liquid films and spray-wall interactions resulting from impinging sprays. International Journal of Heat and Mass Transfer 41(20): 3037-3054.

Stow, C. and M. Hadfield. (1981). An experimental investigation of fluid flow resulting from the impact of a water drop with an unyielding dry surface. Proceedings of the Royal Society of London. A. Mathematical and Physical Sciences 373(1755): 419-441.

Strang, G. (1968). On the construction and comparison of difference schemes. SIAM Journal on Numerical Analysis 5(3): 506-517.

Sussman, M. (2005). A parallelized, adaptive algorithm for multiphase flows in general geometries. Computers \& Structures 83(6-7): 435-444.

Sussman, M., A. S. Almgren, J. B. Bell, P. Colella, L. H. Howell and M. L. Welcome. (1999). An adaptive level set approach for incompressible two-phase flows. Journal of Computational Physics 148(1): 81-124. 
Tan, S. C. (2004). A Tentative Mass Loss Model for Simulating Water Droplet Splash. 42nd AIAA Aerospace Sciences Meeting and Exhibit. Reno, Nevada.

Tanguy, S. and A. Berlemont. (2005). Application of a level set method for simulation of droplet collisions. International journal of multiphase flow 31(9): 1015-1035.

Thoraval, M.-J., K. Takehara, T. G. Etoh, S. Popinet, P. Ray, C. Josserand, S. Zaleski and S. T. Thoroddsen. (2012). von Kármán vortex street within an impacting drop. Physical review letters 108(26): 264506.

Thoroddsen, S. T. (2002). The ejecta sheet generated by the impact of a drop. Journal of Fluid Mechanics 451: 373-381.

Tran, T., H. de Maleprade, C. Sun and D. Lohse. (2013). Air entrainment during impact of droplets on liquid surfaces. Journal of Fluid Mechanics 726.

Tropea, C. and I. V. Roisman. (2000). Modeling of spray impact on solid surfaces. Atomization and sprays 10(3-5).

Trujillo, M., W. Mathews, C. Lee and J. Peters. (2000). Modelling and experiment of impingement and atomization of a liquid spray on a wall. International Journal of Engine Research 1(1): 87-105.

van der Veen, R. C. A., T. Tran, D. Lohse and C. Sun. (2012). Direct measurements of air layer profiles under impacting droplets using high-speed color interferometry. Phys. Rev. E 85: 026315.

Vander Wal, R. L., G. M. Berger and S. D. Mozes. (2006). Droplets splashing upon films of the same fluid of various depths. Experiments in Fluids 40(1): 33-52.

Villedieu, P., P. Trontin and R. Chauvin. (2014). Glaciated and mixed-phase ice accretion modeling using ONERA 2D icing suite. 6th AIAA Atmospheric and Space Environments Conference.

Wang, A. B. and C. C. Chen. (2000). Splashing impact of a single drop onto very thin liquid films. Physics of Fluids 12(9): 2155-2158. 
Weiss, D. A. and A. L. Yarin. (1999). Single drop impact onto liquid films: neck distortion, jetting, tiny bubble entrainment, and crown formation. Journal of Fluid Mechanics 385(1): 229-254.

Weiss, D. A. and A. L. Yarin. (1999). Single drop impact onto liquid films: neck distortion, jetting, tiny bubble entrainment, and crown formation. Journal of Fluid Mechanics 385: 229-254.

White, F. M. (2006). Viscous Fluid Flow, McGraw-Hill Higher Education.

Worthington, A. M. (1908). A Study of Splashes. London: Longmans, Green.

Wright, W. (2008). User's Manual for LEWICE Version 3.2. Cleveland, Ohio, QSS Group, Inc. NASA/CR-2008-214255.

Wright, W. B. (2005). Validation results for LEWICE 3.0. AIAA paper 1243.

Wright, W. B. (2006). Further refinement of the LEWICE SLD model.

Wright, W. B. and M. Potapczuk (1996). Computational simulation of large droplet icing. Proceedings of the FAA International Conference on Aircraft Inflight Icing.

Wright, W. B. and M. G. Potapczuk. (1996). Computational Simulation of Large Droplet Icing. Proceedings of the FAA International Conference on Aircraft In-flight Icing: 545555.

Wright, W. B. and M. G. Potapczuk. (2004). Semi-Empirical Modeling of SLD Physics. NASA/TM-2004-212916.

Wright, W. B. and M. G. Potapczuk (2004). Semi-empirical modeling of SLD physics. AIAA conference, AIAA-2004-0412.

Xu, H., Y. Liu, P. He and H. Wang. (1998). The TAR model for calculation of droplet/wall impingement. Journal of fluids engineering 120(3): 593-597. 
$\mathrm{Xu}$, L., L. Barcos and S. R. Nagel. (2007). Splashing of liquids: Interplay of surface roughness with surrounding gas. Physical Review E 76(6): 066311.

Xu, L., W. W. Zhang and S. R. Nagel. (2005). Drop splashing on a dry smooth surface. Physical review letters 94(18): 184505.

$\mathrm{Xu}, \mathrm{X}$., J. Ouyang, T. Jiang and Q. Li. (2014). Numerical analysis of the impact of two droplets with a liquid film using an incompressible SPH method. $J$ Eng Math 85.

Yarin, A. (2006). Drop impact dynamics: splashing, spreading, receding, bouncing.... Annu. Rev. Fluid Mech. 38: 159-192.

Yarin, A. and D. Weiss. (1995). Impact of drops on solid surfaces: self-similar capillary waves, and splashing as a new type of kinematic discontinuity. Journal of Fluid Mechanics 283(1): 141-173.

Yarin, A. L. (2006). Drop impact dynamics: Splashing, spreading, receding, bouncing... Annual review of fluid mechanics 38: 159-192.

Yarin, A. L. and D. A. Weiss. (1995). Impact of Drops on Solid-Surfaces - Self-Similar Capillary Waves, and Splashing as a New-Type of Kinematic Discontinuity. Journal of Fluid Mechanics 283: 141-173.

Yokoi, K. (2011). Numerical studies of droplet splashing on a dry surface: triggering a splash with the dynamic contact angle. Soft Matter 7(11): 5120-5123.

Yokoi, K., D. Vadillo, J. Hinch and I. Hutchings. (2009). Numerical studies of the influence of the dynamic contact angle on a droplet impacting on a dry surface. PHYSICS OF FLUIDS 21(7).

Zhang, K. and H. Hu. (2014). An Experimental Study of the Wind-Driven Water Droplet/Rivulet Flows over an Airfoil Pertinent to Wind Turbine Icing Phenomena. 4th Joint US-European Fluids Engineering Summer Meeting. Chicago, Illinois, USA.

Zhang, L. V., J. Toole, K. Fezzaa and R. D. Deegan. (2012). Evolution of the ejecta sheet from the impact of a drop with a deep pool. Journal of Fluid Mechanics 690: 5-15. 
Zhao, H.-K., B. Merriman, S. Osher and L. Wang. (1998). Capturing the behavior of bubbles and drops using the variational level set approach. Journal of Computational Physics 143(2): 495-518.

Zhbankova, S. L. and A. V. Kolpakov. (1999). Experimental study of collisions of differently sized droplets. Colloid Journal 61(3): 390-393.

Zheng, L. and H. Zhang. (2000). An adaptive level set method for moving-boundary problems: application to droplet spreading and solidification. Numerical Heat Transfer: Part B: Fundamentals 37(4): 437-454. 


\section{CURRICULUM VITAE \\ Yisen Guo \\ Department of Mechanical Engineering, University of Louisville, Louisville, KY 20292 \\ yisen.guo@louisville.edu}

\section{Education}

- Ph.D. Mechanical Engineering

University of Louisville, Louisville, KY

- $\quad$ M.S. Civil Engineering

Oregon State University, Corvallis, OR

- B.S. Civil Engineering

2009

Shandong University, Shandong, China

\section{Journal Publications}

Guo, Y. and Y. Lian. (2017). "High-Speed Oblique Drop Impact on Thin Liquid Films." Phys. Fluids 29, 082108.

Guo, Y., Y. Lian, and M. Sussman. (2016). "Investigation of Drop Impact on Dry and Wet Surfaces with Consideration of Surrounding Air.” Phys. Fluids 28, 073303.

Li, G., Y. Lian, Y. Guo, M. Jemison, M. Sussman, T. Helms, and M. Arienti. (2015). "Incompressible Multiphase Flow and Encapsulation Simulations Using the Moment-ofFluid Method." International Journal for Numerical Methods in Fluids, 79:456-490.

Guo, Y., D. Trejo, and S.C. Yim. (2015). "New Model for Estimating the Time-Variant Seismic Performance of Corroding RC Bridge Columns." Journal of Structural Engineering, 141 (6), 04014158. 


\section{Conference Proceedings}

Guo, Y. and Y. Lian. (2017). "Investigation of Impact of Multiple Drops on Thin Liquid Film." AIAA Paper 2017-3812.

Guo, Y. and Y. Lian. (2016). "Numerical Investigation of High-Speed Droplet Impact on Solid and Wet Surfaces." AIAA Paper 2016-3276.

Lian, Y. and Y. Guo. (2015). "Investigation of the Splashing Phenomenon of Supercooled Large Droplets." SAE Technical Paper 2015-01-2100.

Lian, Y., Y. Guo, and A. Work. (2014). "Numerical Simulation of Supercooled Large Droplets Using the Moment of Fluid Method" AIAA Paper 2014-0740. 